University of Tennessee Health Science Center

UTHSC Digital Commons

$12-2018$

\title{
Effective Measures of Weight Gain Five Years Post-Kidney Transplantation
}

Tara Calico Cherry

University of Tennessee Health Science Center

Follow this and additional works at: https://dc.uthsc.edu/dissertations

Part of the Cardiovascular Diseases Commons, Endocrine System Diseases Commons, Investigative Techniques Commons, Nutritional and Metabolic Diseases Commons, Other Analytical, Diagnostic and Therapeutic Techniques and Equipment Commons, and the Other Nursing Commons

\section{Recommended Citation}

Cherry, Tara Calico (http://orcid.org/ https://orcid.org/0000-0002-2069-1836), "Effective Measures of Weight Gain Five Years Post-Kidney Transplantation" (2018). Theses and Dissertations (ETD). Paper 468. http://dx.doi.org/10.21007/etd.cghs.2018.0471.

This Dissertation is brought to you for free and open access by the College of Graduate Health Sciences at UTHSC Digital Commons. It has been accepted for inclusion in Theses and Dissertations (ETD) by an authorized administrator of UTHSC Digital Commons. For more information, please contact jwelch30@uthsc.edu. 


\title{
Effective Measures of Weight Gain Five Years Post-Kidney Transplantation
}

\begin{abstract}
BACKGROUND: Weight gain is commonly observed post-kidney transplantation and is associated with unfavorable health outcomes, such as graft loss, new onset diabetes, and cardiovascular disease. The purpose of this study was to determine the most effective measure for assessing body composition after kidney transplantation.
\end{abstract}

DESIGN: The study was a descriptive correlational follow-up study from a single kidney transplant site.

SUBJECTS: A total of 45 eligible patients from a 2007-2011 parent study were selected, ages of 37 to 78 .

MEASUREMENTS: Body composition was obtained 5-8 years posttransplant via anthropometric measures (waist circumference, body mass index, and dual energy X-ray absorptiometry) and compared with baseline (pretransplant) values. Similarly, weight and body mass index (BMI) were obtained. Blood sampling was performed to measure levels of serum glucose, hemoglobin A1C, low-density lipoproteincholesterol, high-density lipoprotein, triglycerides, and coronary risk ratio. Kidney function was monitored via serum creatinine. Manual blood pressure was taken with two resting blood pressures.

RESULTS: The sample size was $N=45$ and included 29 (64.4\%) African Americans and 16 (35.6\%) Whites. There were 25 (55.6\%) males and 20 (44.4\%) females. The ages were 37 to 78 , with a mean of 56 (SD $=10.1$ ). Body weight increased from $186.66 \pm 42.10$ at baseline to $197.89 \pm 48.1$ at the $5-8$ year follow-up, and $\mathrm{BMI}$ increased from $29.03 \pm 4.76$ to $32.14 \pm 9.61$. At the 5-8 year follow-up, anthropometric measure of waist circumference was found to be associated with cardiac risk ratio and weight with diastolic BP. In contrast, 7 body composition measures were associated with HDL, 4 with cardiac risk ratio, 5 with creatinine, and 2 with systolic blood pressure. Significant associations were also found with anthropometric measure BMI obtained at the time of transplant surgery and HDL and LDL 5-8 years later. There were 13 body composition measures associated with HDL, 6 with cardiac risk ratio, and 2 with triglycerides during this same time span. Lastly, one change in anthropometric measure from baseline to 5-8 year posttransplant was associated with the 5-8 year cardiac risk factors (cardiac risk ratio and BMI). There were 18 significant relationships for body compositions measures. These included 7 body composition measures associated with creatinine, 5 with LDL, 4 with diastolic blood pressure, and 2 with triglycerides.

DISCUSSION: The significant relationships found among dual-energy $x$-ray absorptiometry and cardiacrelated outcomes suggest this method may provide a better assessment of body fat, weight gain, and potential cardiac risk factors than does the currently used method. The study continued to examine the use of dual-energy $x$-ray absorptiometry to better understand the emerging coronary risk that accompanies weight gain and as a basis upon which more precisely targeted interventions could be designed that would improve the health and life expectancy of kidney transplant patients.

\section{Document Type}

Dissertation

Degree Name

Doctor of Philosophy (PhD)

\section{Program}

Nursing Science 
Research Advisor

Donna K. Hathaway Ph.D

\section{Keywords}

Body Composition, Cardiovascular, Diabetes, Kidney Transplantation, Obestity, Weight Gain

\section{Subject Categories}

Cardiovascular Diseases | Diseases | Endocrine System Diseases | Investigative Techniques | Medicine and Health Sciences | Nursing | Nutritional and Metabolic Diseases | Other Analytical, Diagnostic and Therapeutic Techniques and Equipment | Other Nursing 
Effective Measures of Weight Gain Five Years Post-Kidney Transplantation

\author{
A Dissertation \\ Presented for \\ The Graduate Studies Council \\ The University of Tennessee \\ Health Science Center
}

In Partial Fulfillment

Of the Requirements for the Degree

Doctor of Philosophy

From The University of Tennessee

\author{
By \\ Tara Calico Cherry \\ December 2018
}


Copyright (C) 2018 by Tara Calico Cherry. All rights reserved. 


\section{DEDICATION}

First, I dedicate this dissertation to "my dad," the late Mr. Robert Lee Calico, who instilled the value of education and fostered my dreams to excel in life no matter how difficult or unrealistic the pathway might become, "keep it moving." His strength and love for education was remarkable until his last day in the classroom, 38 days before his transition from his battle with pancreatic cancer on March 8, 2018. I'm certain he is rejoicing as I write this dedication. Equally, I dedicate my dissertation to my "momma," Anna Black, who supported me in every life event in subtle and effortless fashion. Her silence and willingness to help support my three adopted children made this dissertation possible. Next, I dedicate this dissertation to my children, Roy, Kobe, and Xolani for being unselfish and allowing me to pursue my higher education, by never complaining when I wasn't available physically and emotionally but who embraced the idea I am seeking to make a difference and contribute to our family's legacy of service. I also dedicate this dissertation to my two daughters, Ee'ma and Selah, for inspiring me to find strength in challenges of life and to trust God in all my decisions. I dedicate this dissertation to my grandchildren, Corian, Jakya, Zachary, Roy III, and Ayden for the lessons I learned from each of them. I also would like to dedicate this dissertation to Carolyn Moore, for being supportive during my family's illnesses and deaths over the last year. Finally, but without hesitation,

I dedicate this dissertation to Ruby Anderson, Terri Donald, Patricia Bell Jennings, Terri Oliver, Gregory Strong, and Charles Taylor for their emotional and financial support and strong words of support during my family's near-death illnesses and deaths of my close family members over the last 12 months. I salute my family members who have transitioned from this earth, starting with my late "dad," Robert Lee Calico; my grandma, "Mama" Corine Hampton; my aunt, "Aunt Helen" Helen Carlton; my uncle, "Uncle Bay" Alvin Tuner; my uncle, "Uncle Billy" Billy Neal; and my kindergarten teacher, Miss Harmon, who taught me to love to learn. Finally, I dedicate this dissertation to all Black women and hope to empower them to embrace their fears and challenge themselves to seek opportunities in the obstacles they will face on this earth; I encourage them to find the inner strength beyond the known. 


\section{ACKNOWLEDGEMENTS}

First, I would like to acknowledge Dr. Donna Hathaway for her knowledge, leadership, motivation and emotional support during my family's near-death illnesses and the deaths in my family. I would like to acknowledge my committee members, Dr. Carolyn Graff, Dr. Carrie Harvey, Dr. Tara O'Brien, and Mr. George Relyea, for their support and willingness to share their expertise and resources through my journey. I extend my gratitude to each of my committee members for their time and guidance through the long journey to complete the $\mathrm{PhD}$ program, despite many obstacles. I would like to acknowledge Dr. Carrie Harvey for her commitment to education and her willingness to join the committee without hesitation.

Special acknowledgement is extended to Dr. Hathaway, whose program of research provided the resources that made this dissertation research possible. 


\begin{abstract}
BACKGROUND: Weight gain is commonly observed post-kidney transplantation and is associated with unfavorable health outcomes, such as graft loss, new onset diabetes, and cardiovascular disease. The purpose of this study was to determine the most effective measure for assessing body composition after kidney transplantation.
\end{abstract}

DESIGN: The study was a descriptive correlational follow-up study from a single kidney transplant site.

SUBJECTS: A total of 45 eligible patients from a 2007-2011 parent study were selected, ages of 37 to 78 .

MEASUREMENTS: Body composition was obtained 5-8 years posttransplant via anthropometric measures (waist circumference, body mass index, and dual energy X-ray absorptiometry) and compared with baseline (pretransplant) values. Similarly, weight and body mass index (BMI) were obtained. Blood sampling was performed to measure levels of serum glucose, hemoglobin A1C, low-density lipoprotein-cholesterol, high-density lipoprotein, triglycerides, and coronary risk ratio. Kidney function was monitored via serum creatinine. Manual blood pressure was taken with two resting blood pressures.

RESULTS: The sample size was $N=45$ and included 29 (64.4\%) African Americans and $16(35.6 \%)$ Whites. There were $25(55.6 \%)$ males and $20(44.4 \%)$ females. The ages were 37 to 78 , with a mean of $56(S D=10.1)$. Body weight increased from $186.66 \pm$ 42.10 at baseline to $197.89 \pm 48.1$ at the 5-8 year follow-up, and BMI increased from $29.03 \pm 4.76$ to $32.14 \pm 9.61$. At the 5-8 year follow-up, anthropometric measure of waist circumference was found to be associated with cardiac risk ratio and weight with diastolic BP. In contrast, 7 body composition measures were associated with HDL, 4 with cardiac risk ratio, 5 with creatinine, and 2 with systolic blood pressure. Significant associations were also found with anthropometric measure BMI obtained at the time of transplant surgery and HDL and LDL 5-8 years later. There were 13 body composition measures associated with HDL, 6 with cardiac risk ratio, and 2 with triglycerides during this same time span. Lastly, one change in anthropometric measure from baseline to 5-8 year posttransplant was associated with the 5-8 year cardiac risk factors (cardiac risk ratio and BMI). There were 18 significant relationships for body compositions measures. These included 7 body composition measures associated with creatinine, 5 with LDL, 4 with diastolic blood pressure, and 2 with triglycerides.

DISCUSSION: The significant relationships found among dual-energy $x$-ray absorptiometry and cardiac-related outcomes suggest this method may provide a better assessment of body fat, weight gain, and potential cardiac risk factors than does the currently used method. The study continued to examine the use of dual-energy x-ray absorptiometry to better understand the emerging coronary risk that accompanies weight gain and as a basis upon which more precisely targeted interventions could be designed that would improve the health and life expectancy of kidney transplant patients. 


\section{TABLE OF CONTENTS}

CHAPTER 1. INTRODUCTION .......................................................................................1

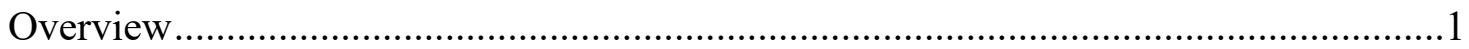

Statement of the Problem..................................................................................... 2

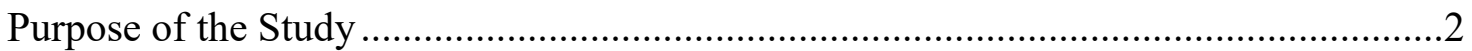

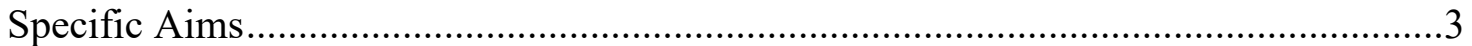

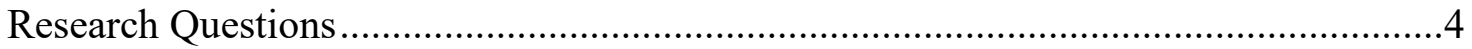

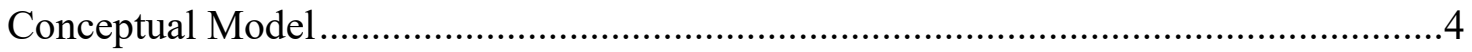

Significance of the Study ...................................................................................... 7

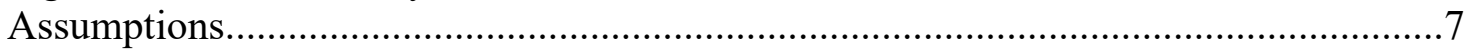

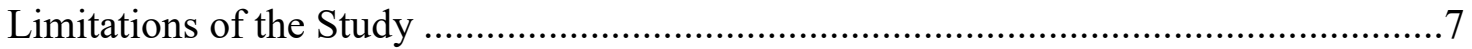

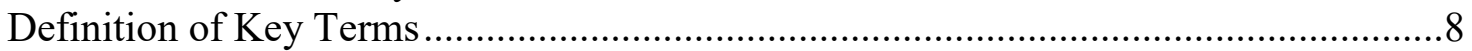

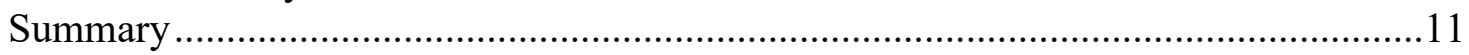

CHAPTER 2. LITERATURE REVIEW ...............................................................13

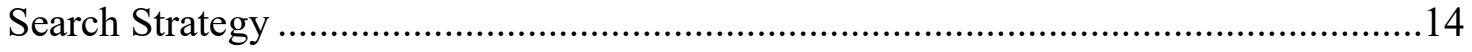

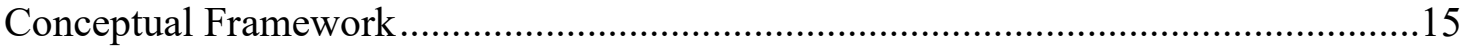

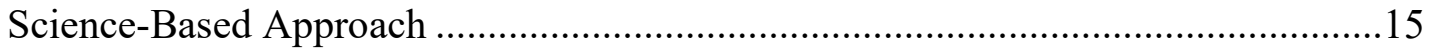

System Approach ...................................................................................... 19

Body Fat in the General Population and Kidney Transplant Population......................20

Weight Gain and Obesity in the General Population..............................................21

Weight Gain and Obesity in the Post-Kidney Transplant Population .........................22

Weight Gain, Obesity Risk, and Cardiovascular Disease Post-Kidney Transplant.......24

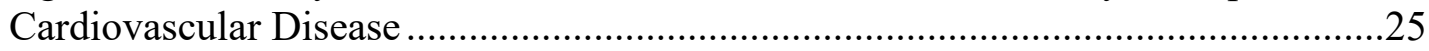

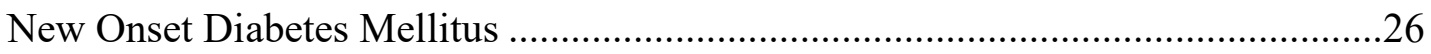

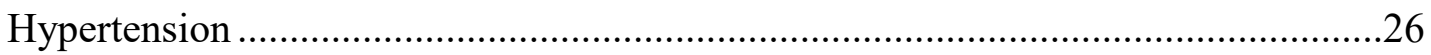

Influence of Posttransplant Fluid and Fat Distribution on Weight Gain ..................27

Donor Source, Gender, Race, and Posttransplant Weight Gain .................................28

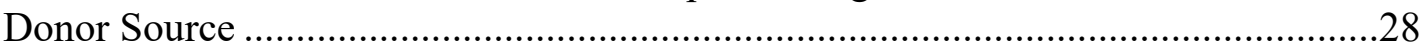

Gender and Race ..............................................................................................28

Other Factors Contributing to Excess Weight Gain in the Kidney Transplant

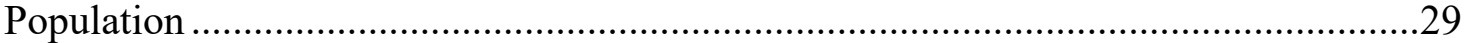

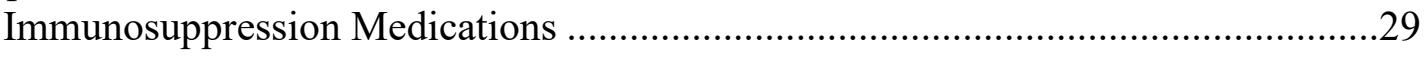

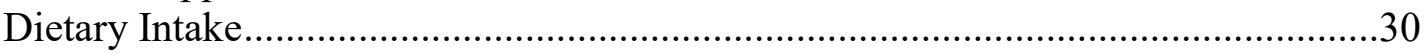

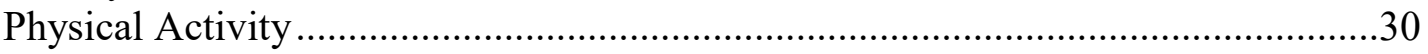

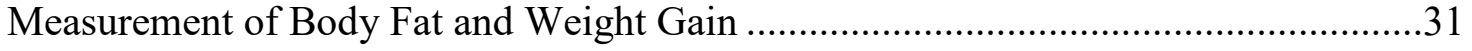

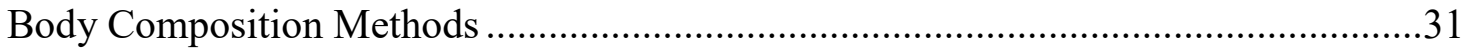

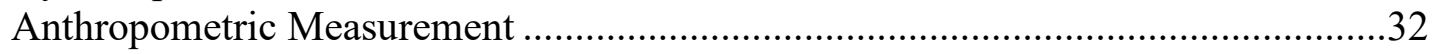

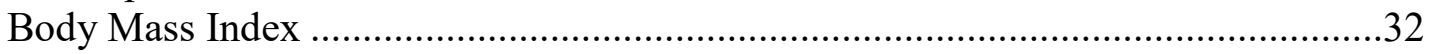

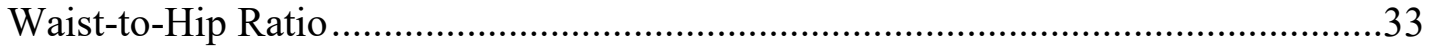

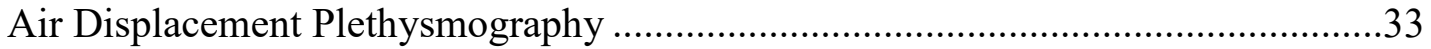

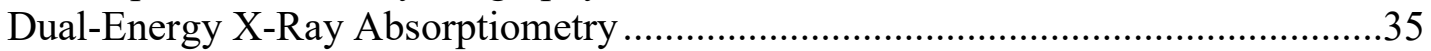

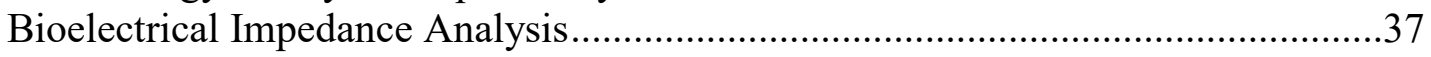

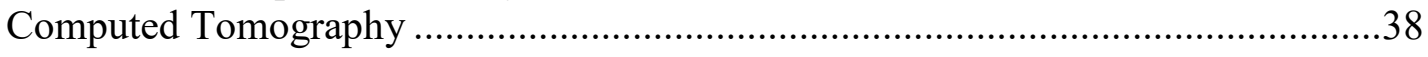




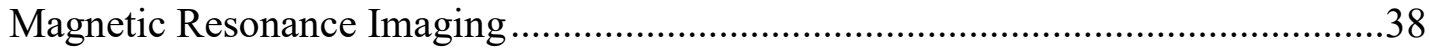

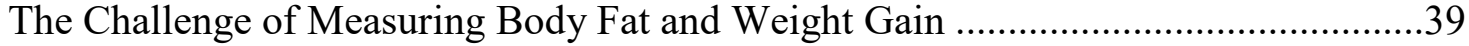

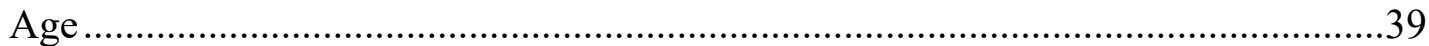

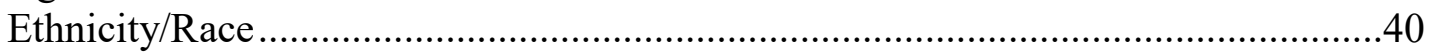

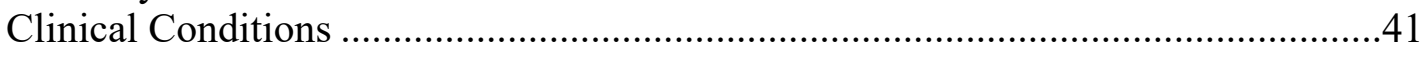

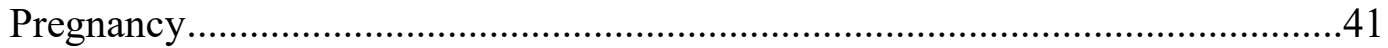

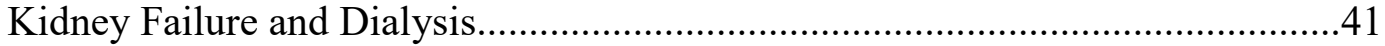

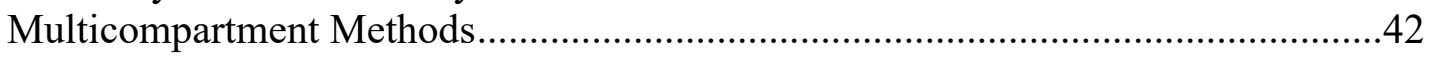

Disease-Specific Population ............................................................................ 45

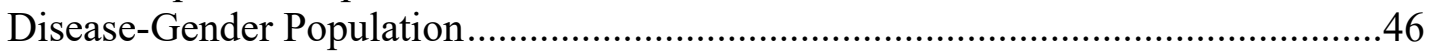

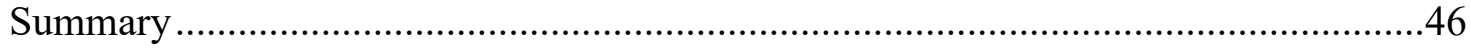

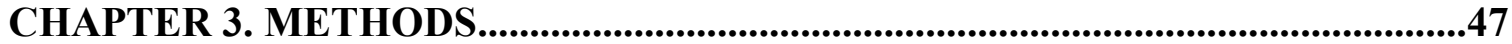

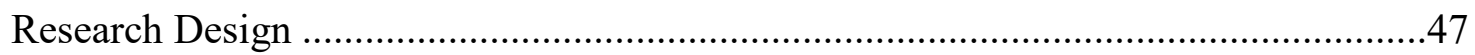

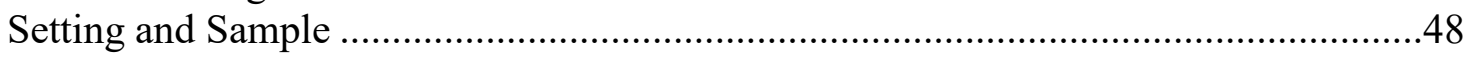

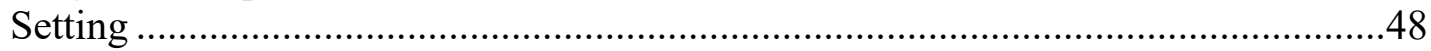

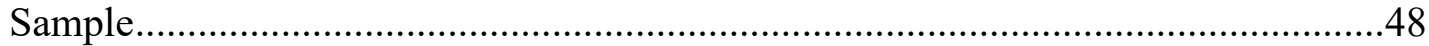

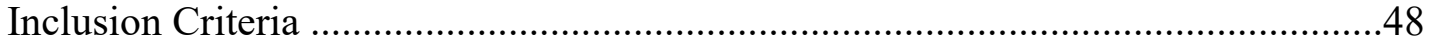

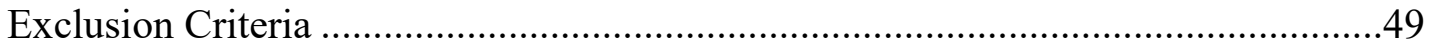

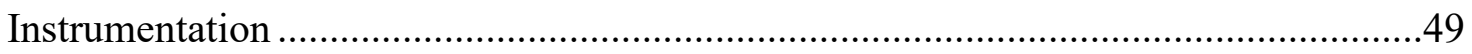

Anthropometric Assessment ......................................................................49

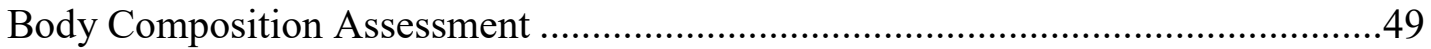

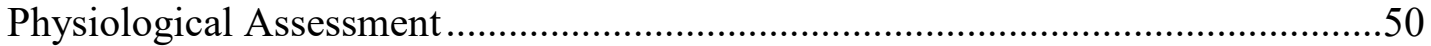

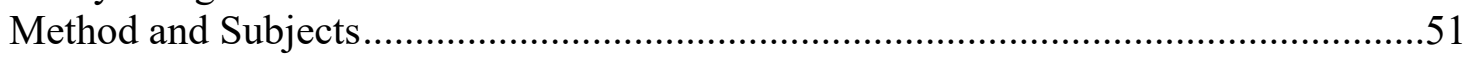

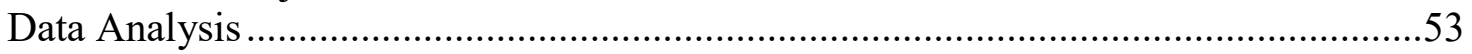

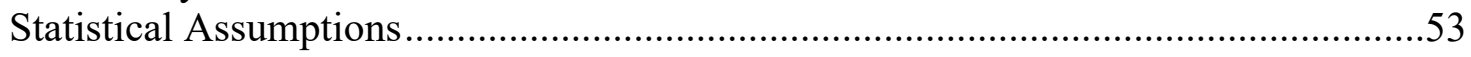

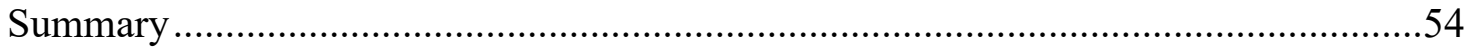

CHAPTER 4. RESULTS ..........................................................................................55

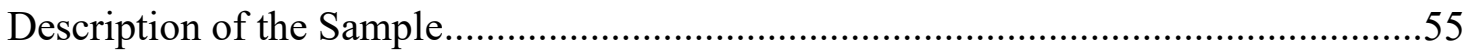

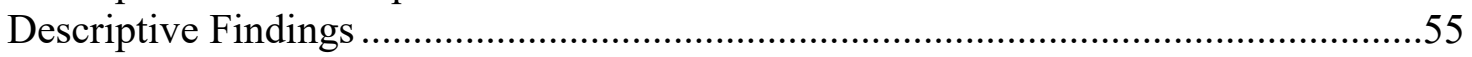

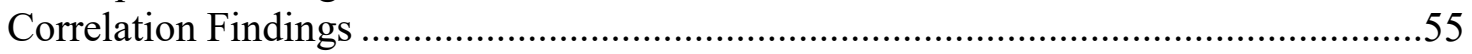

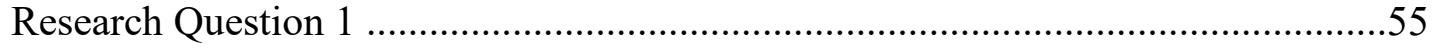

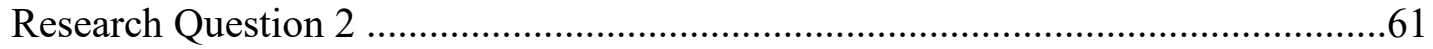

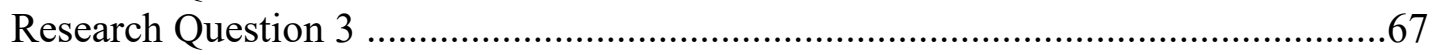

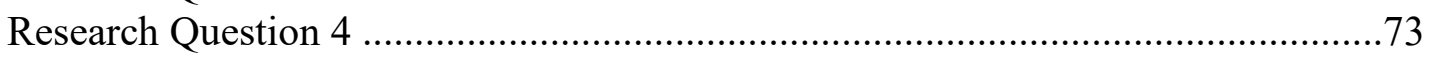

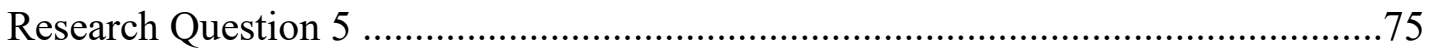

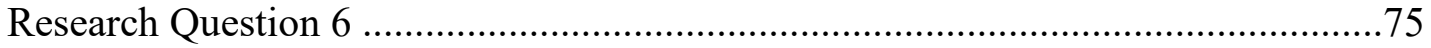

\section{CHAPTER 5. DISCUSSION, CONCLUSION, AND RECOMMENDATIONS.......85}

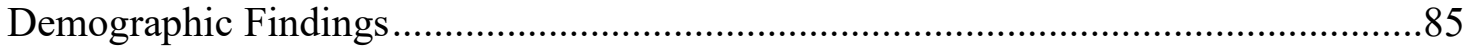

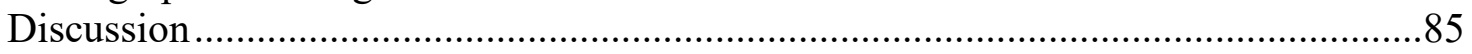

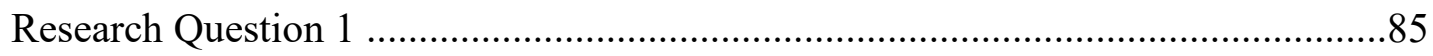

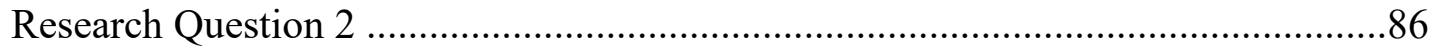

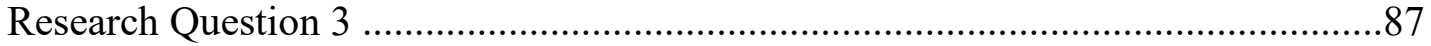

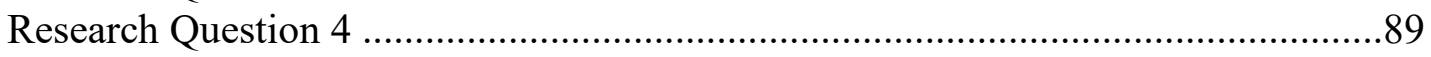




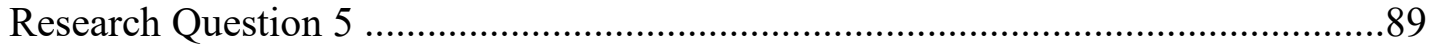

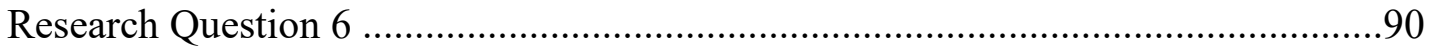

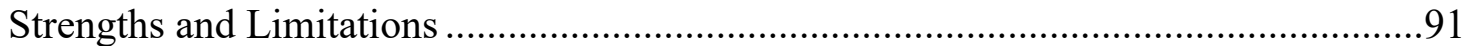

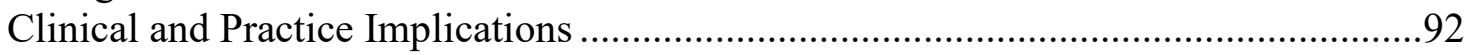

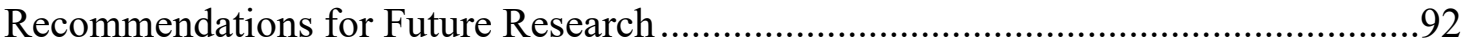

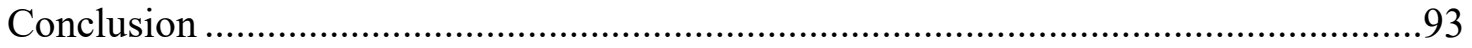

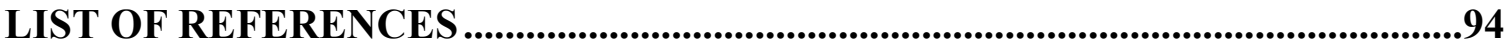

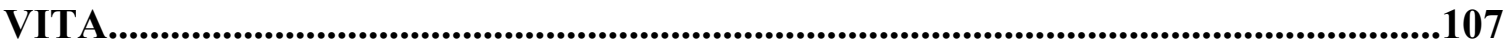




\section{LIST OF TABLES}

Table 2-1. Body Composition Measures and Equations Used to Determine Methods ..17

Table 2-2. World Health Organization Classification of Weight Status by BMI .........23

Table 2-3. Waist-to-Hip Circumference Ratio Norms for Men and Women..................34

Table 2-4. Bioelectrical Impedance Analysis Approaches and Uses ..........................36

Table 3-1 Normal Value of the Blood Sample.........................................................52

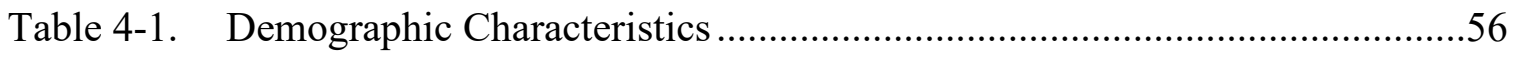

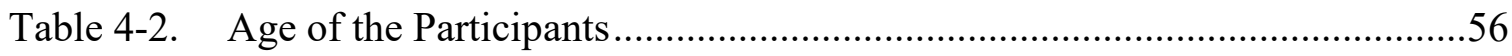

Table 4-3. Anthropometric and Cardiac-Related Outcome Measures at 5-8 Years PostKidney Transplantation ......................................................................56

Table 4-4. Anthropometric, Body Composition, and Cardiac-Related Outcome Measures at Time of Transplant and at 5-8 Years Post-Kidney

Transplantation

Table 4-5. Changes in Anthropometric and Body Composition Measures from Baseline to 5-8 Years Post-Kidney Transplantation

Table 4-6. Relationship Among Cardiac-Related Outcomes and Anthropometric Measures at 5-8 Years Post-Kidney Transplantation.

Table 4-7. Relationship Among Cardiac-Related Outcomes and Body Composition Measures of Total Fat, Total Lean, Total Mass, and Total Percent Fat at 5-8 Years Post-Kidney Transplantation

Table 4-8. Relationship Among Cardiac-Related Outcomes and Body Composition Measures of Android Fat, Android Lean, Android Mass, and Android Percent Fat at 5-8 Years Post-Kidney Transplantation

Table 4-9. Relationship Among Android Gynoid Ratio and Cardiac-Related Outcomes at 5-8 Years Post-Kidney Ttransplantation

Table 4-10. Relationship Among Cardiac-Related Outcome and Body Composition Measures of Gynoid Fat, Gynoid Lean, Gynoid Mass, and Gynoid Percent

Fat at 5-8 Years Post-Kidney Transplantation

Table 4-11. Relationship Among Cardiac-Related Outcome and Body Composition Measures of VFat, VBody Lean, VBody Mass, and VPercent Fat at 5-8 Years Post-Kidney Transplantation 
Table 4-12. Relationship Among Cardiac-Related Outcomes and Body Composition Measures of Whole Body Total Fat, Whole Body Total Lean, Whole Body Total Mass, and Whole Body Total Percent Fat at 5-8 Years Post-Kidney Transplantation.

Table 4-13. Relationship Among Cardiac-Related Outcomes at 5-8 Years Post-Kidney Transplantation and Body Composition Measures of Total Fat, Total Lean, Total Mass, and Total Percent Fat Obtained at Time of Transplant .68

Table 4-14. Relationships Among Cardiac-Related Outcomes at 5-8 Years Post-Kidney Transplantation and Body Composition Measures of Android Fat, Android Lean, Android Mass, and Android Percent Fat Obtained at Time of Transplant

Table 4-15. Relationship Among Cardiac-Related Outcomes at 5-8 Years Post-Kidney Transplantation and Body Composition Measures of Gynoid Fat, Gynoid Lean, Gynoid Mass, and Gynoid Percent Fat Obtained at Time of Transplant

Table 4-16. Relationship Among Cardiac-Related Outcomes at 5-8 Years Post-Kidney Transplantation and Body Composition Measures of Visceral Fat Body Fat, Visceral Fat Body Lean, Visceral Fat Body Mass, and Visceral Fat Body Percent Fat Obtained at Time of Transplant

Table 4-17. Relationship Among Cardiac-Related Outcomes at 5-8 Years Post-Kidney Transplantation and Body Composition Measures of Android Gynoid Ratio, Android Percent Fat, Total Fat Mass, and Fat Mass Ratio Obtained at Time of Transplant .70

Table 4-18. Relationship Among Cardiac-Related Outcomes at 5-8 Years Post-Kidney Transplantation and Body Composition Measures of Whole Body Total Fat, Whole Body Total Lean, Whole Body Total Mass, and Whole Body Total Percent Fat Obtained at Time of Transplant

Table 4-19. Relationship Among Anthropometric Measures of Weight and BMI at Time of Transplant and Cardiac-Related Outcomes at 5-8 Years Post-Kidney Transplantation.

Table 4-20. Relationship Among Cardiac-Related Outcomes at 5-8 Years Post-Kidney Transplantation and Change in Anthropometric Measures of Weight and BMI from Time of Transplant to 5-8 Years Post-Kidney Transplantation..76

Table 4-21. Relationship Among Cardiac-Related Outcomes at 5-8 years Post-Kidney Transplantation and Change in Body Composition Measures of Total Fat, Total Lean, Total Mass, and Total PFat from Time of Transplant to 5-8 Years Post-Kidney Transplantation 
Table 4-22. Relationship Among Cardiac Related Outcomes at 5-8 Years Post-Kidney Transplant and Changes in Body Composition Measures of Android Fat, Android Lean, Android Mass, and Android PF from Time of Transplant to 5-8 Years Post-Kidney Transplantation

Table 4-23. Relationship Among Cardiac-Related Outcomes at 5-8 Years Post-Kidney Transplantation and Changes in Body Composition Measures of Gynoid Fat, Gynoid Lean, Gynoid Mass, and Gynoid PFat from Time of Transplant to 5-8 Years Post-Kidney Transplantation

Table 4-24. Relationship Among Cardiac-Related Outcomes at 5-8 Years Post-Kidney Transplantation and Changes in Body Composition Measures of VFat Body Fat, VFat Body Lean, VFat Body Mass, and VFat Body PFat from Time of Transplant to 5-8 Years Post-Kidney Transplantation .79

Table 4-25. Relationship Among Cardiac-Related Outcomes at 5-8 Years Post-Kidney Transplantation and Changes in Body Composition Measures of Android Gynoid Ratio, Android Percent Fat, Total Fat Mass, and Fat Mass Ratio from Time of Transplant to 5-8 Years Post-Kidney Transplantation .80

Table 4-26. Relationship Among Cardiac-Related Outcomes at 5-8 Years Post-Kidney Transplantation and Change in Body Composition Measures of WBTotal Fat, WBTotal Lean, WBTotal Mass, and WBTotal PFat from Time of Transplant to 5-8 Years Post-Kidney Transplantation 


\section{LIST OF FIGURES}

Figure 1-1. The Body Composition Assessment Model (BCAM). .................................5 


\section{LIST OF ABBREVIATIONS}

\begin{tabular}{ll} 
ADP & Air Displacement Plethysmograph (ADP) \\
AFM & Android Fat Mass \\
A/G & Android/Gynoid Ratio \\
BC & Body Composition \\
BCAM & Body Composition Assessment Model \\
BCM & Body Cell Mass \\
BCV & Body Composition Variation \\
\%BF & Body Fat Percentage \\
BIA & Bioelectrical Impedance Analysis \\
BMD & Bone Mineral Density \\
BMI & Body Mass Index \\
BP & Blood Pressure \\
BV & Body Volume \\
BW & Body Weight \\
CAD & Coronary Artery Disease \\
CDC & Centers for Disease Control and Prevention \\
CKD & Chronic Kidney Disease \\
CNIs & Calcineurin inhibitors \\
CT & Computed Tomography \\
CV & Cardiovascular \\
CVD & Cardiovascular Disease \\
CyA & Cyclosporine A \\
Db & Body Density \\
DBP & Diastolic Blood Pressure \\
DM & Diabetes Mellitus \\
DXA & Dual Energy X-ray Absorptiometry \\
ECW & Extracellular Water \\
ESRD & End-Stage Renal Disease \\
FFBd & Fat-Free Mass Body Density \\
FFM & Fat-Free Mass \\
FFMd & Fat Free Mass Density \\
FM & Total Fat Mass \\
GFM & Gynoid Fat Mass \\
Hb & Hemoglobin \\
HCP & Health Care Professional \\
HD & Hydrodensitometry \\
HDL & High-Density Lipoprotein \\
HTN & Hypertension \\
HW & Hydrostatic Weighing \\
ICW & Intracellular Water \\
KTP & Kidney Transplant Population \\
Kg & Kilogram \\
LBM & Lean Body Mass \\
& \\
\hline
\end{tabular}




\begin{tabular}{|c|c|}
\hline LDL & Low-Density Lipoprotein \\
\hline LM & Lean Mass \\
\hline LRD & Living Renal Donor \\
\hline LTM & Lean Tissue Mass \\
\hline $\mathrm{m}^{2}$ & Meter Square \\
\hline MFBIA & Multifrequency Bioelectrical Impedence Analysis \\
\hline Mo & Total Body Mineral \\
\hline MRI & Magnetic Resonance Imaging \\
\hline NAA & Neutron Activation Analysis \\
\hline NODAT & New Onset Diabetes Mellitus After Transplantation \\
\hline $\mathrm{p}$ & Probability \\
\hline SAT & Subcutaneous Adipose Tissue \\
\hline SBP & Systolic Blood Pressure \\
\hline SKF & Skinfold \\
\hline SFTs & Skinfold Thicknesses \\
\hline STM & Soft Tissue Mass \\
\hline Tac & Tacrolimus \\
\hline TBBM & Total Body Bone Mineral \\
\hline TBCL & Total Body Chloride \\
\hline TBFM & Total Body Fat Mass \\
\hline TBK & Total Body Potassium \\
\hline TBM & Total Body Mineral \\
\hline TBN & Total Body Nitrogen \\
\hline $\mathrm{TBNa}$ & Total Body Sodium \\
\hline TBW & Total Body Water \\
\hline TEM & Technical Error of Measurement \\
\hline TFM & Total Fat Mass \\
\hline TBMD & Total Body Mineral Density \\
\hline UUW & Underwater Weighing \\
\hline VAT & Visceral Adipose Tissue \\
\hline VF & Visceral Fat \\
\hline W & $(\mathrm{kg}) / \mathrm{BW}(\mathrm{kg})$ \\
\hline WBC & Whole Body Composition \\
\hline WBF & Whole Body Fat \\
\hline WC & Waist Circumference \\
\hline WHO & World Health Organization \\
\hline WHR & Waist-to-Hip Ratio \\
\hline
\end{tabular}




\section{CHAPTER 1. INTRODUCTION}

\section{Overview}

Kidney recipients often experience weight gain the first year posttransplant (Baum, 2001 a; Cashion et al., 2007; Jezior et al., 2007). These significant may represent up to $10 \%$ of pretransplant weight (Johnson et al., 1993; Moore \& Gaber, 1996). Aksoy (2016) found that the average weight gain within the first 6 months posttransplant ranged from 6 and $10 \mathrm{~kg}$. Similarly, Cashion et al. (2007) demonstrated mean weight gain ranging from $5-10 \mathrm{~kg}$ within the first year of transplant. These findings are critical, as increases in body weight have been well associated with adverse health outcomes.

Previous studies have explored the association between kidney transplant and weight gain, demonstrating increased morbidity and mortality (Beckmann, Ivanović, Drent, Ruppar, \& De Geest, 2015; Gore et al., 2006). For example, obesity increases kidney sodium reabsorption, which causes hypertension, and excess accumulation of adipose tissue may compress the kidney, which increases intrarenal pressure and tubular reabsorption (Naumnik \& Mysliwiec, 2010). Together, these actions make weight gain following kidney transplantation a serious concern.

The precise etiology responsible for the dramatic weight gain within the postkidney transplant population is poorly understood but likely composed of several contributing factors, including decreased physical activity during the immediate posttransplant course, medically indicated dietary changes, and immunosuppressant therapy. Additionally, weight gain in this population may be attributed (in part) to a global trend toward obesity within developed nations. In the United States since 1960, the overall prevalence of disease associated with obesity has increased across all age, gender, and ethnic groups (Flegal, Carroll, Kuczmarski, \& Johnson, 1998). Today, over $60 \%$ of the United States adult population is classified as overweight or obese, largely from increased adipose stores (Catenacci, Hill, \& Wyatt, 2009).

The link between excess body fat weight and health is well established in the literature (MacLean, Higgins, Giles, Sherk, \& Jackman, 2015). Within the general population, obesity-induced, metabolic syndrome is associated with dyslipidemia, diabetes mellitus, and hypertension, each representing independent risk factors for cardiovascular disease (CVD; Gore et al.,2006). Importantly, kidney transplant recipients are more vulnerable to the physiological changes associated with these diseases (Cashion et al., 2014; Cupples et al., 2012), yielding more severe health consequences. Obesityinduced vasculopathy may also contribute to chronic allograft nephropathy, decreased graft function, and survival with concomitant decreased life expectancy for kidney transplant recipients. This makes monitoring of weight gain and overall body composition critical for these populations.

Several methods of measuring body composition are described in the literature, including single anthropometric or nontraditional technological methods, such as 
bioimpedance analysis (BIA), magnetic resonance imaging (MRI), computerized tomography (CT), air displacement plethysmography (ADP), or dual-energy x-ray absorptiometry (DXA). To date, few studies have explored quantitative methods useful in monitoring body fat composition as a predictor of overall health in post-kidney transplant patients. We propose a novel model of utilizing biomarkers and body composition methods to better identify patients from this subgroup at high risk for body fat weight gain and associated adverse outcomes. Early identification, close continued monitoring, and treatment may greatly improve health outcomes in this population.

\section{Statement of the Problem}

Numerous studies have shown the burdensome nature of postoperative weight gain for kidney transplant patients. This is a common problem and can have negative effects on health outcomes, quality of life, and mortality rates. In particular, excess body fat is a potential risk factor for CVD and other medical conditions (Cashion et al., 2007; Cupples et al., 2012; Gore et al., 2006). The overall problem addressed by our study was the lack of research on the association between changes in body composition (measured by DXA and anthropometric indices) and biomarkers (serum glucose, lipid levels) as predictors of CVD and graft loss after kidney transplant. The specific problem explored was the distribution of body fat and its relationship to diseases. For example, the amount of visceral adipose tissue (VAT) in the thoracic and abdominal cavities has been associated with circulatory disorders and cardiovascular disease. The literature lacks data to determine which methods and measures of weight (anthropometric or DXA) are most accurate in evaluating risk of CVD for kidney transplant recipients.

Although studies have shown that CT, DXA, and MRI provide a direct measure of regional distribution of VAT and have found a strong link between increased abdominal fat and increased morbidity and mortality, anthropometric methods remain the preferred choice for clinicians to assess body composition. Anthropometric methods are commonly preferred due to their relatively low cost and ease of use. The use of anthropometric methods is problematic, however, because of their limited sensitivity or specificity, high variability, and poor correlation with disease to outcome. Consequently, commonly utilized anthropometric approaches provide only a limited assessment of body composition in kidney transplant populations. Thus, the study quantitatively explored the associations of anthropometric and DXA methods with cardiac-related outcomes in a high-risk kidney transplant recipient cohort.

\section{Purpose of the Study}

The primary purpose of this prospective longitudinal correlational study was to investigate if changes in kidney recipients' body composition measured by DXA or anthropometric indices are related to serum glucose, lipid levels, coronary artery ratio, creatinine level, and blood pressure, after 5 years or more. The secondary purpose of this study was to explore the relationship between changes in body composition measures 
assessed by anthropometric measures such as body mass index (BMI), waist circumference (WC), and body weight (BW) and DXA measures of the percentage of body fat \%BF, VAT, total body fat mass (TBFM), android fat mass (AFM), gynoid fat mass (GFM), android/gynoid ratio (A/G), and lean mass (LM) in kidney transplant patients.

It was anticipated that the main aim of this study would help identify ideal anthropometric and/or body composition measures useful in predicting increased cardiovascular risk following kidney transplant surgery. Furthermore, correlating these findings with elevations within serum glucose, lipid levels, coronary artery ratio, creatinine, and blood pressure in kidney transplant recipients could potentially aid in the identification of patients most at risk.

For the purpose of this study, DXA scans were used to exam the distribution of body mass and body fat. To the author's knowledge, no similar studies exist with this population.

\section{Specific Aims}

The overall aim of this study was to compare body composition measures assessed by anthropometric measures and DXA with lab values of serum glucose (glucose and HgbA1C), lipid levels (triglycerides, high-density lipoprotein [HDL], lowdensity lipoprotein [LDL]) and creatinine and blood pressure of kidney transplant patients at 1 and 5 or more years post-kidney transplant. Specifically, the study had six aims:

1. To determine the association between anthropometric measures and serum levels of glucose, lipids, creatinine, and blood pressure of kidney transplant recipients at 5-8 years posttransplant.

2. To determine the association between body composition measures and serum levels of glucose, lipids, creatinine, and blood pressure of kidney transplant recipients at 5-8 years posttransplant.

3. To determine if body composition measures by DXA obtained at the time of transplant (baseline) are associated with serum levels of glucose, lipids, creatinine, and blood pressure of kidney transplant recipients at 5-8 years posttransplant.

4. To determine if anthropometric measures obtained at baseline are associated with serum levels of glucose, lipids, creatinine, and blood pressure of kidney transplant recipients at 5-8 years posttransplant. 
5. To determine the association between changes in anthropometric measures from baseline to 5-8 years posttransplant and serum levels of glucose, lipids, creatinine, and blood pressure in kidney transplant recipients at 5-8 years posttransplant.

6. To determine the association between changes in body composition measures from baseline to 5-8 years posttransplant and serum levels of glucose, lipids, creatinine, and blood pressure at 5-8 years posttransplant.

\section{Research Questions}

Following are the research questions we sought to answer relative to our aims:

RQ1: What is the association between anthropometric measures and serum glucose, lipids, and creatinine levels, and elevated blood pressure in kidney transplant recipients obtained 5-8 years posttransplant?

RQ2: What is the association between body composition measures and serum glucose, lipids, and creatinine levels, and elevated blood pressure obtained in kidney transplant recipients 5-8 years posttransplant?

RQ3: To what degree are body composition measures obtained at the time of transplant (baseline) associated with serum levels of glucose, lipids, and creatinine, and elevated blood pressure in kidney transplant recipients at 5-8 years posttransplant?

RQ4: To what degree are measures of anthropometric obtained at baseline associated with serum levels of glucose, lipids, and creatinine, and elevated blood pressure in kidney transplant recipients at 5-8 years posttransplant?

RQ5: To what degree are changes in anthropometric measures from baseline to 5-8 years posttransplant associated with serum glucose, lipids, and creatinine and elevated blood pressure at 5-8 years posttransplant?

RQ6: To what degree are changes in body composition measures from baseline to 5-8 years posttransplant associated with_serum glucose, lipids, and creatinine, and elevated blood pressure at 5-8 years posttransplant?

\section{Conceptual Model}

The Body Composition Assessment Model (BCAM) was developed following a comprehensive review of the literature to provide direction for this research. This model (Figure 1-1) represents the scientific-technological link between theoretical principles of the human body and the empirical constants in fat-estimating equation models and was used to guide the concepts of methods' and measures' usefulness in clinical settings to 


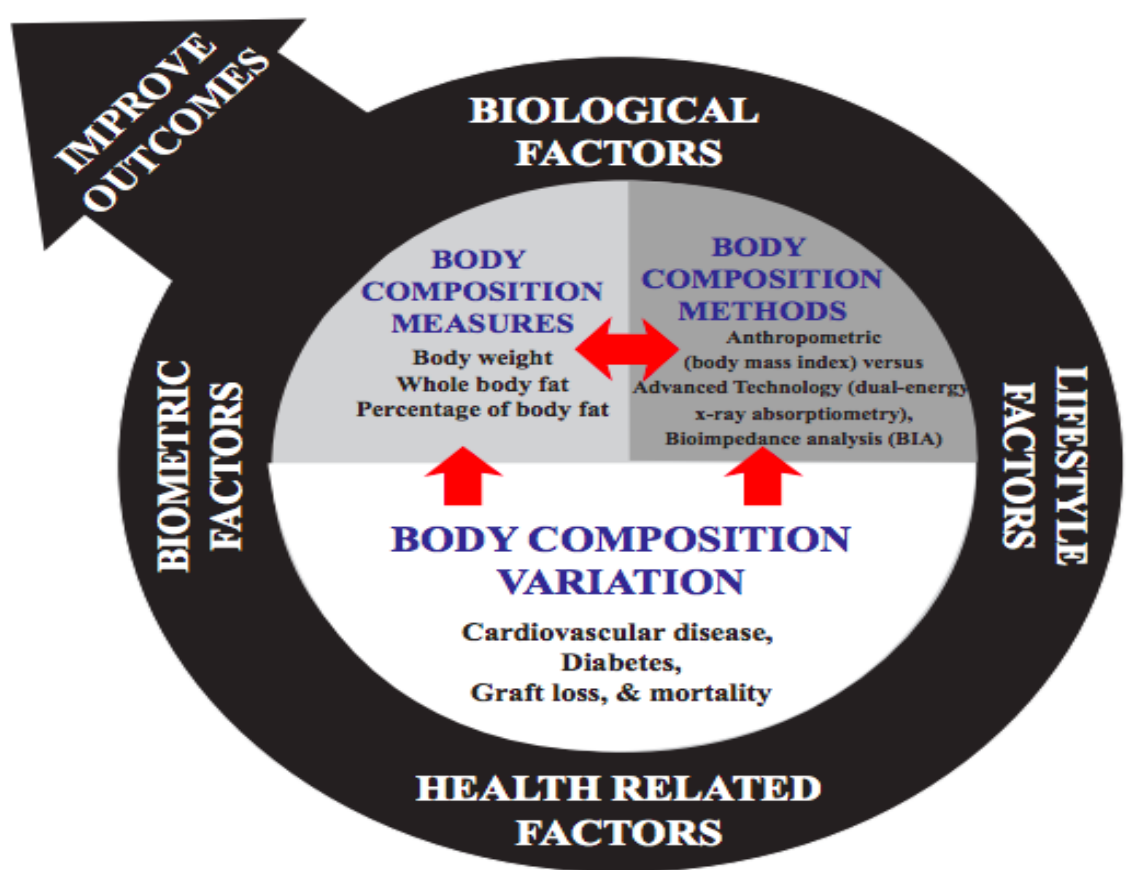

Figure 1-1. The Body Composition Assessment Model (BCAM). 
improve the evaluation of weight gain and improve health outcomes in transplant recipients.

The different factors involved in influencing weight gain and adverse changes in body composition are integrated in the model and represent the effect of overall health outcomes. Commonly used anthropometric, as well as more advanced technological body composition methods such as DXA and BIA, are included in the model-all of which could be used to assess weight and evaluate health outcomes measures in clinical settings. Body composition monitoring, in particular, provides an ideal method of assessing overall health risks in disease states. This strategy provides a noninvasive method of continual evaluation that is well suited for high-risk populations such as kidney transplant recipients. The knowledge obtained through these assessments could prove valuable as a guiding principle and benchmark towards improving health outcomes overall.

In Chapter 2, the conceptual model will be examined more in depth. The next chapter will also discuss an overview of body fat and weight gain in the renal transplant and general population, factors that contribute to weight gain, and the literature on the conditions that transplant patients (renal and otherwise) develop. Of special interest will be the recent history of the use of DXA and the different anthropometric measures used to determine body fat and weight gain. The conceptual model for this study is illustrated in Figure 1-1.

The BCAM is a systems-based model that helps health care professionals in the selection of the most accurate method to evaluate changes in body composition, thereby improving monitoring of patient outcomes in clinical settings. Ideally, the use of the BCAM model will bridge the gap in understanding the linkage between body composition physiology and selection of the most suitable measures for monitoring the process of variation in body composition. This will be done specifically by acknowledging the interaction among body compositional changes that occur during weight gain, aging, the presence or absencs of diseases, and variation in body composition that exist among ethnic groups. Selection of the more appropriate measure is critical for use of the correct marker for monitoring risk for chronic diseases and to evaluate health outcomes.

The BCAM provides an approach to better understand the role of body composition in kidney transplant populations and identify the best methods for assessing body composition. Clinicians and researchers will gain knowledge of appropriate body composition methods and measures for assessing weight gain in the kidney transplant population. In addition, future research programs can use and expand upon the BCAM to advance methods used to assess body composition in kidney recipients, yielding improved health outcomes. 


\section{Significance of the Study}

The outcome of this study will contribute to our long-term goal of establishing clinically useful measures that can reliably identify patients at risk of experiencing cardiovascular health complications following kidney transplant surgery. Cardiovascular complications are the leading cause of graft failure and death in this population. The findings of this study could potentially improve the health and life expectancy of kidney transplant patients by providing data that could be used to help design and monitor the efficacy of new treatment modalities, particularly for those patients who experience substantial posttransplant weight gain. The significance of this study could help increase long-term survival rates for kidney transplant patients, improve their quality of life, and provide a means by which they can receive better care, diagnoses, and prognoses.

\section{Assumptions}

This study assumed that the outcome measures would be reflective of the individuals' cardiac risk status.

\section{Limitations of the Study}

Several limitations were present that should be considered when examining the study results:

1. Use of a single site may not be generalizable to other populations; however, the study site serves as a diverse representation of the general transplant population.

2. Study measures were taken at different times and by different individuals. However, all measures were validated and standardized, with the same protocol and equipment used at all measurement points.

3. Use of a single posttransplant time point approximately 5 years or longer posttransplant may limit utility of study findings. For example, when weight gain occurs at 3-4 years or 6-8 years, other medical conditions could worsen along with CVD risk. However, previous studies suggest these comorbidities generally occur during the early years following transplant surgery.

4. Only measures of body fat and specific aspects of blood chemistry were considered even though there are many other factors associated with CVD risk for kidney recipients and in the general population. However, those used in this study are stronger predictors and most commonly used in clinical settings. 


\section{Definition of Key Terms}

Substantial literature provides consistent definitions for variables and major concepts, especially scientific concepts. Key terms and definitions we used during our research follow:

Abdominal obesity. The excessive subcutaneous and visceral fat locate in the abdominal region also known as upper-body obesity (Heyward \& Wagner, 2004).

Adipose tissue. Body fat that is approximately $83 \%, 2 \%$ protein, and $15 \%$ water (Heymsfield, Lohman, Wang, \& Going, 2005).

Air displacement plethysmography (ADP): Air displacement method measures body volume and body density to assess body composition (Heyward \& Wagner, 2004).

Anthropometry. The science of measuring body size and proportion (Heyward \& Wagner, 2004).

Android fat mass (AFM). The amount of adipose tissue in the abdominal area and below the individual neck. It is derived from the fat tissue in the android region in grams (Tanamas et al., 2012).

Android obesity. Identified by localized excess body fat found in the upper-body; it is also known as an apple-shaped body (Heyward \& Wagner, 2004).

Android/Gynoid Ratio. Android fat is the amount of fat between the bottom of an individual's head and the top of the iliac crest in the lowest $20 \%$ region. Gynoid fat is the amount of fat located downward from the android region and from the top of the greater trochanter. It is derived from percentage of fat in the android region divided by percentage of fat in the gynoid region (Fu et al., 2014).

Bioelectrical impedance analysis (BIA). A body composition method used to measure total body water and fat-free mass (Dehghan \& Merchant, 2008).

Body composition. Body composition refers to the partitioning of body makeup, including protein, fat, water, and minerals fat-mass (weight or percentage) and fatfree mass weight (Heymsfield et al., 2005).

Body density (Db). The measure of the total body mass to total body volume (Heyward \& Wagner, 2004).

Body mass (BM). Body weight; total size of the body (Heyward \& Wagner, 2004). 
Bone mineral content (BMC). The measure of mineral in bones by the DXA method that is independent of the body composition calibration method selected (Heyward \& Wagner, 2004).

Cardiovascular disease (CVD). Cardiovascular diseases are disorders involving the heart and blood vessels, such as hypertension, coronary artery disease, cardiac dysrhythmias, cerebrovascular disease, heart failure, peripheral vascular disease, and congenital cardiac abnormalities (World Health Organization [WHO], 2018).

Dual energy x-ray absorptiometry (DXA). Refers to a method used in clinical and research settings to estimate bone mineral density, bone mineral content, fat, and lean soft tissue mass (Heymsfield, Wang, Baumgartner, \& Ross, 1997).

Essential lipids. These compound lipids are important for cell membrane formation and make up a small portion of the body the total lipid (Heyward \& Wagner, 2004).

Fat-free mass (FFM) density or fat-free body (FFB) density. The measure of lipid-free chemicals and tissues found in internal organs, connective tissue, muscle, bone, and water (Heyward \& Wagner, 2004).

Fat mass (FM) density. The measure of lipids found in adipose tissue and other tissues (Heyward \& Wagner, 2004).

Four-component models (4-C). 4-C models refers to the development of technological advances in measuring based on water, minerals, protein, and fat components in the body at the molecular level (Heyward \& Wagner, 2004).

Gynoid fat mass (GFM). The amount of fat in the lower portion of an individual's body around the hip area. It is derived from the fat tissue in the gynoid region in grams (Heyward \& Wagner, 2004).

Gynoid obesity. Identified by localized excess body fat found in lower body fat; pear-shaped. It is known also called pear-shaped body (Heyward \& Wagner, 2004).

Healthy body weight. A body weight that does not increase the possibility of developing a disease risk (Heyward \& Wagner, 2004).

Hydrometry. A human body measurement of water (Heyward \& Wagner, 2004).

Hydrodensitometry, also called hydrostatic weighing (HW) or underwater weighing (UUW). A method used to estimate total body volume submerging a person's body into water (Heyward \& Wagner, 2004). This is a good measure of body volume. 
Magnetic resonance imaging (MRI). "Technique used to create computerized crosssectional images of the human body radio frequency signals emitted by hydrogen nuclei" (Heyward \& Wagner, 2004, p. 230).

Multifrequency BIA (MFBIA). "A BIA approach in which a wide range of frequencies $(1 \mathrm{kHz}$ to $1 \mathrm{MHz})$ are used to estimate extracellular, intracellular, and total body water:

Also known as bioelectrical" (Heyward \& Wagner, 2004, p. 230).

Multicomponent models. A model composed of three or more components that is used to develop advanced technology methods and measurements based on variation in water, mineral, and protein in the fat-free mass of the human body (Heyward \& Wagner, 2004).

Nonessential lipids. Triglycerides found in the adipose tissue in the human body (Heyward \& Wagner, 2004).

Percentage of body fat or relative body fat (\%BF). \%BF is an individual total amount of fat divided by total body weight Percent Fat (android + gynoid regions). \% $\mathrm{BF}=100 \times($ Fat Mass $) /$ Mass. \%BF will be lower for the "classic" calibration (Heymsfield et al., 2005).

Reference method. Refers to direct measures of human body components that are used and considered "gold standard" in the development of models, methods, and prediction equations for assessing body compositions (Heyward \& Wagner, 2004).

Six-component models (6-C). 6-C models refer to the development of technological advances based on direct measure of chemical composition in vivo of six components in the body: water, calcium, potassium, sodium, nitrogen, and chloride (Heyward \& Wagner, 2004).

Three-component models (3-C). 3-C models refer to the development of technological advances based on water, minerals, and tissues components in the body. These components are divided into three levels called 3-C water molecular level, 3-C mineral molecular level, 3-C cellular level, or 3-C tissue level (Heyward \& Wagner, 2004).

Total body water (TBW). The total amount of extracellular and intracellular fluid found in the different areas of the body (Heyward \& Wagner, 2004)

Total body bone mineral (TBBM). A measure of all the bone mineral content in the body (Heyward \& Wagner, 2004).

Total body mineral (TBM). The amount of mineral found in the human body bone and cells (Heyward \& Wagner, 2004). 
Total bone mineral density (TBMD). The measure of mineral in bone and cells measured by DXA and for this study, is independent of the body composition calibration method selected of the 4500 HolgicA equipment (Heyward \& Wagner, 2004).

Total fat mass (TFM). An indirect measurement of body fat that is estimated from total body water or the mean density of the whole body. The fat mass is measured by DXA in grams Android + Gynoid regions. Fat mass measures depend on the Body Composition Calibration Method (Heyward \& Wagner, 2004).

Total lean tissue mass (TLM). The total body mass less the bones and fat in an individual's body total lean mass in the Android +Gynoid regions. It derived from the lean standard deviation value. This is known as the soft tissue component of "FatFree" Mass. Lean Tissue Mass $=$ Mass - Fat mass - BMC (Heyward \& Wagner, 2004).

Two-component models (2-C). 2-C models are divided into FM and LBM and assume these components are consistent for all individuals (Heyward \& Wagner, 2004).

Visceral Adipose Tissue (VAT). This is the amount of fat tissue surrounding an individual's organs that impacts a wide variety of clinical risk factors including fasting glucose levels, serum triglycerides, and cholesterol (Bergman et al., 2006).

Waist circumference (WC). "Measure of central adiposity and upper-body obesity; waist girth" (Heyward \& Wagner, 2004, p. 232).

Waist-to-hip ratio (WHR). Waist circumference divided by the hip circumference; used "as a measure of upper-body obesity and visceral fat" (Heyward \& Wagner, 2004, p. 232).

Whole-body fat. Total body fat measurements provide useful physiological and other medical conditions information. They are measured in grams by DXA and include all fat tissues in all regions including extractable lipids from adipose tissue and other tissues. Also called total body fat mass (TBFM; (Heyward \& Wagner, 2004).

For other important abbreviations used in this document, see the List of Abbreviations in the front matter.

\section{Summary}

This prospective correlational study attempted to examine the association of various methods and measures of body composition and serum glucose, lipid levels, coronary artery ratio, creatinine, and blood pressure associated with weight gain in 
kidney transplant patients. The population included individuals who had undergone kidney transplant procedures 5-8 years prior to this study and had participated in the parent study. Current blood work was obtained from these individuals to determine serum glucose, lipid, and creatinine levels.

This chapter outlined the goals and purpose of the study used to address the research problem and gain knowledge regarding the best measures to assess changes in body composition that occur during weight gain in kidney transplant recipients. The translation of this knowledge will bridge the gap in research and clinical settings by identifying accurate measures to evaluate the risk of CVD. The significance of the research lies with the potential of how the study's results may impact further research and practice in the monitoring and treatment of kidney transplant recipients, as well as others who are in high cardiac risk groups.

Chapter 2 will provide an overview of the relevant literature, including support for the conceptual framework, an overview of the CVD and weight gain problems experienced by kidney and other transplant patients, and a review of studies on body composition methods and measures. 


\section{CHAPTER 2. LITERATURE REVIEW}

Kidney transplantation has been the preferred choice of treatment for patients with end-stage renal disease (ESRD) and is the most commonly performed organ transplantation. However, after transplant, a number of recipients experience short-term and long-term complications (Urstad, Wahl, Andersen, Oyen, \& Fagermoen, 2012). Short- term complications typically occur in the immediate postoperative course and include edema, kidney dysfunction, pain, and respiratory insufficiency (Elster et al., 2008). Powerful immunosuppressive therapies have enabled prolonged survival enlarging subpopulations that develop long-term complications. Thus, long-term complications pose the greatest source for morbidity and mortality in posttransplant recipients. This makes identification and management of long-term complications critical. One important long-term complication is weight gain. Increased fat mass accumulation contributing to obesity is a serious concern in kidney transplant recipients. This chapter will briefly review effects of adipose accumulation on health, morbidity, and mortality in renal transplant recipients. This is followed by an overview of existing methods available to quantitate body composition, focusing on those most likely to accurately predict well-being within this study population.

Body composition assessments vary in precision and in the target tissue (s) of interest. Both the target body composition tissue (s) such as visceral adipose or android tissue and the patient health conditions are key players for assessing body composition. Both are important phenomenon to address to guide clinicians to use anthropometric methods with cautions. These commonly used methods, such as BMI, WC, and WHR do not provide suitable body composition measures, specifically body fat, which is critical to health outcome. The continuous and frequent use of these methods is due to its quick, simple, and inexpensive use. Clinicians should caution the use of these anthropometric methods with the general population, as well as in the kidney transplant population due to possible health threats for people in this country. The continuous use of body composition methods that yield unsuitable body composition measures may potentially increase morbidity and mortality rate in kidney recipients (Lee \& Gallagher, 2008).

The link between obesity and chronic disease is well established in the literature, negatively impacting individuals across all ages, races, gender, and ethnic groups. The literature also begs this question: Why is BMI considered an appropriate method for assessing body composition and evaluating obesity-related risk in the healthy and unhealthy population, in the face of a continuous rise in the obesity epidemic?

The literature in this chapter will also explore reasons why simply tracking body weight is neither adequate for assessing body composition, nor suitable for identifying risk factors associate with weight gain. In addition, it is important for clinicians to understand how biological and pathological factors such as age, ethnicity, gender, and presence or absence of diseases influence changes in body composition versus height and weight. For this reason, the literature will discuss advantages and disadvantages of 
appropriate advanced technology methods and anthropometric methods in the clinical setting.

The literature strongly guides and illustrates the need to conduct the study effective measures of weight gain 5 years post-kidney transplantation. In addition, the literature outlines numerous influential factors that alter body compositional changes in the human body, however, the literature lacks a consensus on the most suitable body composition methods and measures. The literature also provides the foundation for development of the BCAM platform, which aids selection of the most effective method and measure for measuring weight, particularly for individuals with chronic conditions such as kidney recipients.

This chapter presents a review of literature related to the presence and risks associated with weight gain and obesity, and methods employed to measure body fat and weight gain. The first section describes the search strategy used to identify literature for this review followed by sections that discuss the conceptual model, body fat in the general population and kidney transplant population, weight gain and obesity in the postkidney transplant population in the factors contributing to weight gain and obesity in the kidney transplant population, measurement of body fat and weight gain; and the challenges of measuring body fat and weight gain.

\section{Search Strategy}

The search strategy for this review used primary sources (e.g., World Health Organization) and secondary sources (e.g., research method books) from 1991-2018. The search yield was sorted by scientific rigor and relevance to the topic area. Relevant articles were identified, and MeSh terms were used to identify other credible and relevant articles. Endnote and Refworks were used for reference mangers. Relevant reviews and studies were obtained from the UTHSC library database using PubMed Central, PubMed Health, and Web of Science. Searches also included database such as CINHAL Complete, JAMA, JSTOR, Medline Plus, and Goggle Scholar. Searches were limited to studies on humans, adult kidney transplant recipients, single organ transplant, and studies published in English. Unpublished studies were not reviewed.

The search also included systematic reviews and meta-analyses, literature reviews, and other types of research using Boolean search terms that included body composition measures and methods, body composition and weight gain, weight gain and kidney transplant population, obesity and kidney transplant population, weight gain and obesity and kidney transplant population, risk factors and kidney transplant population, body fat and kidney transplant population, over weight and obesity and kidney transplant population, complications and kidney transplant population, immunosuppression medications and kidney transplant population, cardiovascular disease and kidney transplant population, dietary intake and physical activity and kidney transplant population, measurement and body fat and weight gain, anthropometric measures and instrumental scans, DXA and BIA methods and measures, MRI, CT, ADP and body 
composition assessment, body composition and cardiovascular disease, kidney transplant population, and the measurement of weight and obesity.

Studies were considered for inclusion whether they were observational, crosssectional, cohort, longitudinal, prospective and retrospectives, or comparative. The participants of the studies included adult kidney/kidney transplant recipients and adults who used methodological measurement to assess health outcomes, and predictions were included. Recipients of any transplant other than a kidney transplant were excluded, including kidney-pancreas transplant recipients.

The literature search was repeated multiple times using these same search terms prior to publication of the parent study's findings, and likewise throughout the preparation for, conduct of, and reporting of results from this follow-up study. New relevant evidence was found in this search and was included in the current study.

\section{Conceptual Framework}

The Body Composition Assessment Model (BCAM) is a novel approach that incorporates dynamic factors in guiding clinicians to appropriate body composition analysis, tailored to the unique individual. BCAM provides a conceptual framework for assessing body composition and may prove beneficial in better identifying specific etiology for weight gain. This model comprises both system and scientific concepts (Figure 1-1). System functions involve biological, biometric, health-related (disease), and lifestyle factors. These four concepts influence body compositional changes, either directly or indirectly during active weight gain or in the presence of chronic diseases, as detailed below.

Additionally, there are three science-based concepts involving body composition measure, body composition variation, and body composition methods which can be used as a platform to accurately select a body composition method. For example, kidney transplant recipients who develop complications of kidney dysfunction are prone to fluid imbalance and may develop fluid shifts from third spacing and fluid overload. Weight gain from fluid imbalance is difficult to differentiate from weight gain due to other sources, such as fat accumulation. BCAM could be utilized in this setting to guide clinicians in selection of appropriate body composition test to better guide treatment.

\section{Science-Based Approach}

Science-based approach concepts are organized into three interconnected areas within the center of the BCAM framework called body composition measure, body composition method, and body composition variation. Within the clinical setting these factors are routinely assessed during routine visits. In the following paragraphs, the author will review these concepts in detail. 
First, body composition variation is the largest science-based concept because it is a key player in the determination of method selection in this framework. Typically, this is the first concept assessed during routine clinic visits. Body fat within adult populations is distributed within whole body and regional locations and comprised of visceral adipose and subcutaneous fat deposits. Variations in subcutaneous fat can be readily apparent during physical exam. Patients may exhibit characteristic android or gynoid fat distribution patterns (defined in body composition assessment section). Visceral adipose tissue (VAT) describes fat deposits surrounding visceral organ tissue and is more difficult to assess and quantitate. For example, visceral fat kills healthy patients. Patients that have undergone kidney transplant are more susceptible to alterations within visceral fat stores. One underlying assumption in the BCAM is the ongoing direct and/or indirect relationship between each concept in the system-science based approach with body composition variation for determining appropriate measures.

Secondly, body composition measures quantify the relationship between the effective measures and suitable methods. This is an important step to determining the most effective measure to assess body composition. Components of body composition are quantified from fat mass (FM), fat-free mass (FFM), protein, mineral, or bone density (Db) measurements. These measurements are mathematically calculated to estimate body composition measures such WBF, VF, AFM, GFM, A/G Ratio, and LM (Heymsfield et al., 1997). The most effective measure to assess body composition is determined by body composition models and predictive equations. Each model estimates measures such as $\% \mathrm{BF}, \mathrm{WBF}, \mathrm{FM}, \mathrm{FFM}, \mathrm{AFM}, \mathrm{GFM}$, and $\mathrm{A} / \mathrm{G}$ ratio from a mathematical equation based on known or unknown component-, property-based, or combined-based models (Table 2-1) to develop suitable predictive equations to design effective body composition methods. For example, according to Heyward and Wagner (2004) a known componentbased method (i.e., hydration, Db) measures \%BF from FM divided by BW x100 (see Table 2-1, Heymsfield, et al., 2005). Clinicians' careful application of the model's principles and assumptions are essential to effective measures of weight gain in kidney recipients.

Lastly, the appropriate method selection is determined by the available measures of three types of body composition methods referred to as component-, property-, and combined-based methods. The three methods are the platform for determining the most appropriate measure and the best method to evaluate body composition in a kidney transplant population or a general population.

The author argues for population specificity body composition methods based on disease, gender, age, ethnicity, or physical status are critical to effectively assess weight gain. Predictive equations are useful in diverse populations as well as a specific population where alteration in fat distribution and fluid disturbances are common. A 4-C molecular model should not be considered in kidney transplant recipients or any population with a chronic disease who may experience fluid disturbance with electrolyte imbalance. The four elements of the 4-C model are fat, protein, bone mineral, and total 
Table 2-1. Body Composition Measures and Equations Used to Determine Methods

\begin{tabular}{|c|c|c|c|}
\hline Level & Model & Body Equation & Reference \\
\hline $\begin{array}{l}\text { Two-Component } \\
\text { Molecular Level }\end{array}$ & $\begin{array}{l}\mathrm{BW}=\text { fat }+ \text { fat free } \\
\text { body }\end{array}$ & $\begin{array}{l}\% \mathrm{BF}=[(4.57 / \mathrm{Db})- \\
4.142] \times 100 \\
\% \mathrm{BF}=[(4.95 / \mathrm{Db})- \\
4.50] \times 100\end{array}$ & $\begin{array}{l}\text { Brozek, } 1963 \\
\text { SirI, } 1956\end{array}$ \\
\hline \multirow[t]{2}{*}{$\begin{array}{l}\text { Three- } \\
\text { Component } \\
\text { Molecular Level }\end{array}$} & $\begin{array}{l}\mathrm{BW}=\text { fat }+ \text { water }+ \\
\text { (mineral and protein } \\
\text { combined) }\end{array}$ & $\begin{array}{l}\% \mathrm{BF}=[(2.118 / \mathrm{Db}) \\
-0.78 \mathrm{~W}-1.354] \mathrm{x} \\
100\end{array}$ & Siri, 1961 \\
\hline & $\begin{array}{l}\mathrm{BW}=\text { fat }+ \text { mineral } \\
+(\text { water and protein } \\
\text { combined })\end{array}$ & $\begin{array}{l}\% \mathrm{BF}=[(6.386 / \mathrm{Db})- \\
3.961 \mathrm{M}-6.090] \mathrm{x} \\
100\end{array}$ & Lohman, 1986 \\
\hline $\begin{array}{l}\text { Three- } \\
\text { Component } \\
\text { Tissue Level } \\
\text { (DXA) }\end{array}$ & $\begin{array}{l}\mathrm{BW}=\text { bone }+ \text { bone- } \\
\text { free lean tissue fat }\end{array}$ & $\begin{array}{l}\% \mathrm{BF}=\mathrm{FM} / \mathrm{BW} \mathrm{x} \\
100\end{array}$ & Ellis, 2000 \\
\hline $\begin{array}{l}\text { Four-Component } \\
\text { Molecular Level }\end{array}$ & $\begin{array}{l}\mathrm{BW}=\text { fat }+ \text { water }+ \\
\text { bone mineral }+ \\
\text { protein }\end{array}$ & $\begin{array}{l}\% \mathrm{BF}=[(2.559 / \mathrm{Db}) \\
-0.734 \mathrm{~W}+0.983 \mathrm{~B}- \\
1.841] \times 100 \\
\% \mathrm{BF}=[(2.747 / \mathrm{Db}) \\
-0.714 \mathrm{~W}+1.146 \mathrm{~B}- \\
2.053] \times 100 \\
\% \mathrm{BF}=[(2.513 / \mathrm{Db}) \\
-0.739 \mathrm{~W}+0.947 \mathrm{~B}- \\
1.790] \times 100 \\
\% \mathrm{BF}=[2.747 / \mathrm{Db})- \\
0.718 \mathrm{~W}+1.148 \mathrm{~B}- \\
2.050] \times 100\end{array}$ & $\begin{array}{l}\text { Selinger, } 1977 \\
\text { Heymsfield, } 1996\end{array}$ \\
\hline $\begin{array}{l}\text { Six-Component } \\
\text { Atomic Level }\end{array}$ & $\begin{array}{l}\mathrm{BW}=\mathrm{TBW}+\mathrm{TBN} \\
+\mathrm{TBCa}+\mathrm{TBK}+ \\
\mathrm{TBNa}+\mathrm{TBCI}\end{array}$ & $\begin{array}{l}\mathrm{FM}(\mathrm{kg})=\mathrm{BW}- \\
(\mathrm{TBW}+6.525 \mathrm{TBN} \\
+2.709 \mathrm{TBCa}+2.76 \\
\mathrm{TBK}+\mathrm{TBNa}+1.43 \\
\mathrm{TBCI})\end{array}$ & Wang et al., 1998 \\
\hline
\end{tabular}

Note. $\% \mathrm{BF}=$ relative body fat; $\mathrm{Db}=$ total body density $(\mathrm{g} / \mathrm{cc}) ; \mathrm{FM}=$ fat mass $(\mathrm{kg}) ; \mathrm{W}=$ $(\mathrm{kg}) / \mathrm{BW}(\mathrm{kg})$, where $\mathrm{TBW}=$ total body water and $\mathrm{BW}=$ body weight; $\mathrm{M}=\mathrm{TBM}=$ total body mineral (osseous + cell mineral) and $\mathrm{BW}=$ body weight; $\mathrm{B}=\mathrm{TBBM}(\mathrm{kg}) / \mathrm{BW}(\mathrm{kg})$, where $\mathrm{TBBM}=$ total body bone mineral (osseous mineral only) and $\mathrm{BW}=$ body weight; 


\section{Table 2-1. Continued.}

Constant: $\mathrm{TBBM}=$ bone ash $\times 1.0436 ; \mathrm{TBM}=$ bone ash $\times 1279 ; \mathrm{TBN}=$ total body nitrogen; $\mathrm{TBCa}=$ total body calcium; $\mathrm{TBK}=$ total body potassium; $\mathrm{TBNa}=$ total body sodium; $\mathrm{TBCL}=$ total body chloride.

Note. Reprinted with permission from author Dale R. Wagner 9-16-18. Originally published in "Applied body composition assessment", by Heyward, V. H., \& Wagner, D. (2004), p. 11; out of print. 
body water (Heyward \& Wagner, 2004). The multi-compartment (4-C) model is a highly significant predictor of the relationship between age and ethnicities. For example, Asian populations have a high level of adipose tissue, especially in the abdominal area and 4-C model would be a better estimate of body fat than an anthropometric method such as BMI or WC. The use of anthropometric method such as BMI in an Asian population may prolong diagnosing individuals who are at risk for cardiovascular disease. Multicomponent models are generally thought to provide more accurate estimates of body fat than two-component models, especially when one of the assumptions of the two-component model might be violated, such as constant hydration, which is not likely in the kidney transplant population (Heyward \& Wagner, 2004).

\section{System Approach}

The BCAM is a system approach for assessment of body composition in the clinical setting. The system concept describes the function of biological, health-related, biometric, and lifestyle factors in guiding optimal body composition assessment in healthy or unhealthy populations. In the BCAM (Figure 1-1), biological factors influence body composition variation. First, it is important to understand the body composition changes at the cellular, the molecular level, and tissue-organ level when determining the most effective measure for assessing body composition (Baumgartner, Heymsfield, Lichtman, Wang, \& Pierson, 1991; Gao et al., 2008) and the underlying assumptions used to estimate $\%$ BF. For example, health-related factors such as metabolic syndrome, hyperlipidemia, hypertension, diabetes, cardiovascular disease, graft loss, and mortality (Anjana et al., 2004) alter body composition changes at the cellular, molecular, or tissueorgan level and during active weight gain.

The health-related concept is essential for determining the most suitable measure for assessing body composition, and the presence of a specific disease during active weight gain. For example, kidney recipients often experience fluid disturbance and accumulation of body fat with increased body weight. As a result, weight gain could alter fat distribution in different anatomical areas and cause disturbance intra- and extracellular fluid (Heyward \& Wagner, 2004). Both variations in body composition conditions may cause health-related conditions such as cardiovascular disease or new onset diabetes. A clinician could apply the presence of a health-related condition to determine the best measure for assessing body composition in a patient with alteration in fluid disturbance or distribution of fat. Next, the biometric factors in the BCAM represent the relationship between anthropometric methods and whole body composition (WBC). The anthropometric variables are measurable biometric factors used to calculate BMI, WC, WHR, and skinfold (SKF) methods. These methods include measures of body weight, body volume, body surface, waist, hip, thigh, arm, bi-iliac, knee, ankle, elbow, height, and recumbent length. The BCAM describes a direct or indirect relationship between biometric factors and each factor within the system-based approach and one concept science-based approach called body composition variation. For example, the BCAM lifestyle factors such as decreased physical activities and increased caloric intake may 
indirectly influence body composition variation by altering fat distribution throughout the body.

Collectively, the BCAM concepts and factors demonstrate a direct and/or indirect interaction between a systematic-science-based approach that helps to identify accurate measures of body composition to determine the most effective method for assessing body composition. The understanding of the relationship between components of body composition and biological and pathological impact on body composition changes is key to accurate measures and methods and improving health outcomes.

\section{Body Fat in the General Population and Kidney Transplant Population}

Body fat is the fat found in adipose tissue, which is stored below the skin and surrounding the internal organs (Heymsfield, Lohman, Wang, Going, 2005). Percent $\mathrm{BF}$ is an estimate of total body fatness used to evaluate the relationship between excess body weight, accumulation of adipose tissue, and health (Teixeira, Sardinha, Going, \& Lohman, 2001)). Historically, studies have shown a relationship between BMI, \%BF, and metabolic complications of adiposity, elevated lipoproteins, and cardiovascular disease (Despres et al., 1990; Kissebah et al., 1982; Teixeira et al., 2001). More critical findings by Kwakernaak, Toering, and Navis (2013) suggested numerous factors that contribute to health complications were also associated with excess weight measured by BMI. Health complications such as chronic kidney disease were strongly linked to central body fat distribution.

Numerous studies have identified limits in BMI guidelines and variation in measures in population specificity. For example, some studies found a correlation between accumulation of visceral fat in the abdominal cavity elevates triglycerides, reduces high-density lipoprotein cholesterol, elevates blood pressure, and elevates fasting plasma glucose and measures of body fat by BMI or adiposity (Cofan, Vela, \& Cleries, 2005; Ojo et al., 2000). Cofan et al. (2005) found weight gain after transplantation contributed to the prevalence of obesity, with women significantly higher than men $(21 \%$ vs. $13 \% ; P<0.0001)$. Numerous studies found a link between increased adiposity, $\% \mathrm{BF}$, BMI, and CVD risk factors. However, BMI category and the excessive abdominal fat in Asians and Europeans were largely different and the Europeans were more susceptible to CVD and obesity-related health diseases (Gill, 2001).

Another study reported a significant increase in body fat and BMI among women African American women, 12 months following kidney transplantation (Pantik, Cho, Hathaway, Tolley, \& Cashion, 2017). Another author found Asians with a low BMI had a higher percentage of fat compared to Caucasians and African Americans, showing BMI is an unreliable predictor of mortality risk Gallagher et al. (2000a).

There is substantial literature to support BMI limitations, specifically the inability to accurately assess body fat and predict health outcomes in a diverse population. This prompts an urgency for future research to address continuous pitfalls of BMI use and 
challenges for the reduction in prevalence of morbidity and mortality found among weight gainers. In addition, an appropriate future consideration is the identification of population-specific body composition measures based on age, gender, ethnicity, race, physical activity, and presence and absence of a disease.

\section{Weight Gain and Obesity in the General Population}

According to WHO (2018), the prevalence of overweight and obese adults has tripled and continues to rise in the general population. In 2016, over 1.9 billion adults were categorized as overweight and 650 million were considered obese. This was approximately $13 \%$ of the world's adult population and $15 \%$ women and $11 \%$ men. Within this population, it was reported that $39 \%$ of the adults were overweight and $13 \%$ obese with an estimated 35.5\% women and 32.5\% men (WHO, 2018).

More alarming, by 2030, if the current trend continues to rise at a steady rate, the future prevalence of BMI measures in the unhealthy category is projected at $86.6 \%$ in the adult population. More specifically, Black women (96.9\%) and Mexican American women $(91.1 \%)$ will be most affected in the adult population. The study also suggested by 2048, all adults' BMI measures could be classified as unhealthy, and by 2034, Black women will be among the first group to reach unhealthy BMI measures (Wang, Beydoun, Liang, Caballero, \& Kumanyika, 2008). Another study projected 65 million more obese adults in this country by 2030 (Wang, McPherson, Marsh, Gortmaker, \& Brown, 2011). According to the Centers for Disease Control and Prevention (CDC, 2014), the obesity trend differs among racial and ethnic groups, and for adults ages 20 or older is as follows:

1. Hispanic population $8.5 \%$ for Central and South Americans, 9.3\% for Cubans, 13.9\% for Mexican Americans, and 14.8\% for Puerto Ricans;

2. Asian American population including Chinese (4.4\%), Filipinos (11.3\%), Asian Indians (13.0\%);

3. American Indian (8.8\%) and Alaska Natives (6.0\%), and 24.1\% of the American Indians in southern Arizona.

The CDC (2014) also reported the prevalence of diabetes was expected to rise based on prediabetes cases in 2009-2012. In the U.S., the prediabetes cases are different between ethnic and racial groups. The percentages of nonHispanic Whites, nonHispanic blacks, and Hispanics diagnosed with prediabetes were 35\%, 39\%, and 38\%, respectively. In 2012, 37\% of the prediabetes cases in the U.S. were adults ages 20 or older, and 51\% ages 65 or older. The total number of prediabetes cases for the American population was $86,000,000$ for ages 20 and over. Monitoring of body composition and weight gain should be examined to include measures more suitable for estimating body fat and identifying health risk. 
BMI is commonly used to classify individuals based on abnormal or excess accumulation of fat (Beckmann, Ivanović, Drent, Ruppar, \& De Geest, 2015). WHO defines healthy BMI measures as 18.5 to 24.9 , while unhealthy BMI measures range from $\geq 25$ to $\geq 30$. The unhealthy ranges are categorized as overweight and obese, as shown in

Table 2-2.

Regardless of the exact percentage of increase, the rising rate of obesity in the United States and worldwide has serious ramifications for the health of the population and concomitant demands on the health care system, due to the associated chronic conditions accompanying excessive weight gain. For example, short-term complications from significant weight gain and obesity are delays in wound healing and wound infection (Zrim, Furlong, Grace, \& Meade, 2012), while long-term complications include development of chronic diseases such as Type 2 diabetes, CVD, hypertension (HTN), and other comorbidities (Lafranca, IJermans, Betjes, \& Dor, 2015a; Silkensen, 2000). Adversely, the rise in the prevalence of weight gain and unhealthy BMI measures in adults yields an increased burden in several diseases such a cardiovascular diseases and diabetes. The classification for BMI is shown in Table 2-2 (DiCecco \& Francisco-Ziller, 2014).

\section{Weight Gain and Obesity in the Post-Kidney Transplant Population}

Obesity is a common problem in the post-kidney transplant population. However, more critical is the accumulation of excess weight gain in areas that specifically contribute to the prevalence of life-threatening clinical and subclinical diseases. The severity and complications of these diseases are associated with the quantity and distribution of body fat in the kidney recipients. In addition, the increase in body fat may influence fluid disturbance and increase distribution of visceral adipose tissue in the body. As a result, kidney recipients may develop NODAT or CVD (Cashion et al., 2007). DM and CVD whether preexisting or new-onset following transplantation are common diseases diagnosed in transplant recipients and cause life-threatening health complications (Calò et al., 2017; Neale \& Smith, 2015; Ogden, Carroll, Kit, \& Flegal, 2013; Paripovic, Kostic, Spasojevic, Kruscic, \& Peco-Antic, 2010b).

The precise extent to which obesity affects the health of the general population is uncertain; however, it is known that weight gain influences changes in body compositions that are magnified in kidney transplant recipients. While weight gain and obesity are risk factors for hyperlipidemia, hypertension, atherosclerosis, and decreased life expectancy in the general population, these threats are exacerbated for kidney transplant recipients and accompanied by additional risks for allograft nephropathy and graft loss. It is also noteworthy that the enhanced CVD risk following transplant is manifested by nearly all recipients with experiences of elevated blood pressure, high lowdensity lipoprotein and low high-density lipoprotein-cholesterol levels (elevated total cholesterol), elevated insulin levels and blood glucose, and abnormal blood lipids. Given the prevalence of chronic health conditions that are typically associated with obesity, 
Table 2-2. World Health Organization Classification of Weight Status by BMI

\begin{tabular}{lc}
\hline Weight Status & Body Mass Index, $\mathbf{~ k g} / \mathbf{m}^{\mathbf{2}}$ \\
\hline Underweight & $<18.5$ \\
Normal Weight & $18.5-24.9$ \\
Overweight & $25-29.9$ \\
Obese & \\
Class I obesity & $30-34.9$ \\
Class II obesity & $35-39.9$ \\
Class III obesity & $>49$ \\
\hline
\end{tabular}

Note: Adapted with permission from World Health Organization (2018). Global Database on Body Mass Index, 2018. Retrieved from

http://www.assessmentpsychology.com/icbmi.htm 
abdominal (visceral) adiposity is undoubtedly responsible for cardiovascular-related events being the leading cause of graft loss. In addition to increasing the risk for CVD, visceral adiposity has also been identified as a risk factor for DM and specifically associated with insulin resistance, dyslipidemia, and hyperinsulinemia (Banerji, Faridi, Atluri, Chaiken, \& Lebovitz, 1999).

Excessive body weight and obesity pose significant serious threats to health outcomes of transplant recipients compared to individuals in the general population (Beckmann et al., 2015). Typically, a weight gain of $10 \mathrm{~kg}$ occurs during the first year following transplant surgery and may double to $32 \mathrm{~kg}$ after the first year (Beckmann et al., 2015; Cashion et al., 2007; Cupples et al., 2012; Lentine et al., 2008; Stanfill et al., 2015). Consequently, the incidence of short-and long-term health complications associated with weight gain is higher in the transplant population compared to the general population (Beckmann, Ivanović, Drent, Ruppar, \& De Geest, 2015). In addition, several studies have reported that the average weight gain that occurs following transplant surgery is an independent risk factor for graft loss (Cashion et al., 2007; Jezior et al., 2007; Stanfill, Bloodworth, \& Cashion, 2012) and serves as a negative predictor of patient survival (Gore et al., 2006).

The following sections will discuss weight gain and obesity risk on cardiovascular disease post-kidney transplant recipients; the influences of posttransplant fluid and fat distribution on their weight; and the relationships among donor source, gender, race, and posttransplant weight gain.

\section{Weight Gain, Obesity Risk, and Cardiovascular Disease Post-Kidney Transplant}

The evidence of health complications associated with excess body weight and the prevalence of increased body weight and fat following kidney transplantation remains a constant, as well as a serious impact on health conditions. In the United States, since 1960, the overall prevalence of a metabolic diseases has been associated with increased weight gain across all age, gender, and ethnic groups (Flegal et al., 1998).

More critically, weight gain leads to progressive changes in the quantity and distribution of certain compartments of body composition such as visceral adipose tissue (VAT) and often results in two major diseases including CVD and Type 2 diabetes (Cashion et al., 2007; Fernandes et al., 2013; Lafranca, Ijermans, Betjes, \& Dor, 2015b). Additionally, there is convincing evidence that VAT volume, rather than subcutaneous fat, is correlated with the presence of other CVD risk factors such as dyslipidemia and hyperinsulinemia (Banerji et al., 1999).

The risk of CVD associated with weight gain highlights the importance of monitoring and periodically measuring changes in body composition in the kidney transplant population. A study by Rao and Coates (2018) found an association between CVD risk factors such as hypertension and dyslipidemia and diabetes following kidney transplantation. Alshehri (2010) found that measures such as abdominal adiposity, 
elevated blood pressure, high low-density lipoprotein and low high-density lipoproteincholesterol levels (elevated total cholesterol), elevated insulin and blood glucose, and abnormal blood lipids were significant markers for cardiovascular risk and other health complications.

For the above reasons, this study explores the association between markers of CVD identified in the literature and body composition measured by anthropometrics and DXA (Baum, 2001b; Fernandes et al., 2013; Lafranca et al., 2015b; Rao \& Coates, 2018). As studies have shown, individuals with extreme levels of body fat are at greater risk for developing CVD (Shah \& Braverman, 2012). Therefore, a goal of this study was to better understand the effective monitoring of body weight and measures, and to evaluate the level of body fat that falls at or near extreme levels that could result in serious health problems and effectively reduce quality of life in kidney transplant recipients.

\section{Cardiovascular Disease}

CVD is one of the leading causes of mortality and long-term morbidity in kidney transplant recipients (Fernandes et al., 2013; Lentine et al., 2010; Neale \& Smith, 2015). The number of deaths due to CVD was estimated at $36 \%$ for the kidney transplant population (Elli, Traversi, \& Ponticelli, 2000). On the other hand, in general, a prospective study in 2007 estimated the deaths associated with CVD at $66 \%$ in individuals with central obesity compared to $44 \%$ with non-central obesity (Orazio et al., 2007), while the estimated deaths in the after kidney transplant population ranged from $30 \%$ to $50 \%$ (Dimeny, 2002). Following transplantation, CVD risk is manifested by more than half of the recipients experiencing elevated BP, high low-density lipoprotein (LDL), low high-density lipoprotein (HDL), elevated total cholesterol, elevated insulin levels and blood glucose, and abnormal blood lipids (Dimeny, 2002; Elli et al., 2000; Neale \& Smith, 2015). These health conditions, typically associated with excess abdominal (visceral) adiposity, are susceptible markers for CVD, which is one of the leading causes of allograft loss (Neale \& Smith, 2015).

Patients with CKD and on dialysis have a higher risk of developing CVD (Marcén, 2006; Neale \& Smith, 2015) compared to the general population (Jun, Lv, Perkovic, \& Jardine, 2011). Although kidney transplantation reduces the recipients' risk of cardiovascular (CV) events (Neale \& Smith, 2015), the presence of excess fat increases the patients' risk of developing CVD (Armstrong, Campbell, Hawley, Johnson, \& Isbel, 2005; Baum, 2001b; Cashion et al., 2007; Chan, Garneau, \& Hajjar, 2015; Cordeiro et al., 2013; Dimeny, 2002; Fernandes et al., 2013; Lafranca et al., 2015a) or worsening CV events (Armstrong et al., 2005). CVD can lead to morbidity and mortality for posttransplant patients (Marcén, 2006), dialysis patients, and CKD patients as an individual's kidney function continues to decline (Steiber, 2014). 


\section{New Onset Diabetes Mellitus}

According to Ogden, Carroll, Kit, and Flegal (2014), more than one third of U.S. adults $(34.9 \%)$ are obese. On the other hand, Eckel et al. (2011) stated that $34 \%$ of the U.S. adult population is obese, with more than $11 \%$ of individuals over the age of 20 diagnosed with diabetes and the number is expected to increase $21 \%$ by 2050 . Many individuals who are obese will develop Type II diabetes (Eckel et al., 2011). Eckel et al. (2011) stated that a connection between obesity and Type II diabetes exists, but the connection remains unclear.

Diabetes is diagnosed with excess blood glucose within the body (Shivaswamy, Boerner, \& Larsen, 2016) and may lead to kidney failure (Ogden et al., 2013). DM is diagnosed in $3 \%$ to $20 \%$ of the kidney transplant population (Baum, 2001a). Historically, in 2011-2012, 36.5\% of the U.S. chronic kidney disease adults aged 20 years or older were diagnosed with diabetes based on fasting elevated blood sugar levels or HgbA1C (CDC, 2017). Similarly, in 2015, the CDC estimated, 30.2 million people are affected with diabetes, which represents 7.2 million of the U.S. population who are undiagnosed with diabetes. Prediabetes is found in $48.3 \%$ of adults aged 65 years or older. Also in 2015, approximately, 84.1 million American adults were diagnosed with prediabetes based on HgbA1C (CDC, 2017).

Consequently, if the prevalence of excess weight gain in the kidney transplant population continues to rise (Kwan, Hajjiri, Metwally, Finn, \& Perkins, 2016), it is possible the prevalence of new onset diabetes mellitus after transplantation (NODAT) will continue to rise (Olyaei, deMattos, \& Bennett, 1999; Shivaswamy et al., 2016; Yu et al., 2016b). Approximately $4 \%$ to $25 \%$ kidney transplant recipients will develop NODAT (Peev, Reiser, \& Alachkar, 2014). NODAT can result in graft loss and poor survival rates for kidney recipients (Kwan et al., 2016; Yu et al., 2016a). Obesity pre and posttransplantation may worsen NODAT (Peev et al., 2014; Yu et al., 2016a), especially since several immunosuppression medications to reduce transplant rejection increase diabetogenic potential (Peev et al., 2014).

New onset diabetes mellitus following transplantation (NODAT) occurs in the presence of excessive weight gain and increased triglyceride levels following transplant surgery (Olyaei et al., 1999; Pham, Pham, Pham, Pham, \& Pham, 2011; Shivaswamy et al., 2016; Yu et al., 2016a); (Kim et al., 2013). In addition, up to 50\% of kidney transplant recipients are reported to develop NODAT (Pham et al 2011), which often presents severe, life-threatening comorbidities.

\section{Hypertension}

According to Paripovic, Kostic, Spasojevic, Kruscic, and Peco-Antic (2010a), suggested hypertension is a serious and common problem after kidney transplantation; therefore, early intervention should begin immediately after surgery. The acute and chronic complications experienced after kidney transplant are critical to survivability of 
the allograft; therefore, new onset HTN should be avoided after kidney transplant. With respect to HTN guidelines, formerly, HTN was classified as BP above $140 \mathrm{mmHg}$ systolic blood pressure (SBP) and above $90 \mathrm{mmHg}$ diastolic blood pressure (DBP) (Mafutha \& Wright, 2013). However, the Whelton et al. (2018) guidelines identify two stages to classify HTN: Stage I defines HTN as SBP $>130-139 \mathrm{mmHg}$ and DBP $>80-89$ mmHg. However, stage two defines HTN as SBP $>140 \mathrm{mmHg}$ and DBP $>90$ $\mathrm{mmHg}$. Azancot et al. (2015) found an association between hypertension and subclinical inflammation and atherosclerosis 24 hours after kidney transplant with elevated SBP $(p<.0001)$. Another study found $50 \%$ to $90 \%$ of the kidney transplant population were diagnosed with HTN (Fernandes et al., 2013).

There are several factors that contribute to HTN in the kidney transplant population, which are more critical when patients gain weight while taking immunosuppressive medications. First, a high percentage of transplant recipients experience a sedentary lifestyle, which contributes to the obesity, thus influences the onset of HTN (Neale \& Smith, 2015; Paripovic et al., 2010b). Secondly, the life-long use of immunosuppression after kidney transplantation, such as corticosteroids and calcineurin inhibitors (CNIs), may trigger elevated blood pressure and HTN (Calò et al., 2017; Neale \& Smith, 2015; Paripovic et al., 2010b). Next, the use of corticosteroids by kidney recipients may cause hypernatremia that could lead fluid disturbance, such as fluid retention. Unfortunately, fluid retention is a common problem in the kidney transplant population, which may trigger elevated BP, leading to HTN (Tantisattamo, 2017). Lastly, the use of CNIs prevents acute organ rejection and prolongs graft survival (Hoorn et al., 2012; Kalluri \& Hardinger, 2012; McPartland \& Pomposelli, 2007), yielding favorable post-kidney transplant outcomes. Lastly, the use of CNIs causes vasoconstriction in the kidney and often induces HTN (Hoorn et al., 2012).

\section{Influence of Posttransplant Fluid and Fat Distribution on Weight Gain}

Heyward and Wagner (2004) stated that fluid disturbance influences body compositional changes and is altered by weight gain; specifically, declines in lean body mass and total body water (TBW) lead to increased total body fat. The decline in TBW

and a rigorous medication regimen following kidney transplantation can induce a severe fluid imbalance and additional complications, such as congestive heart failure, poor kidney function, insulin resistance, ascites, and edema. For example, kidney transplant recipients routinely take medications from several drug classes, which influences symptomatic changes, such as fluid retention or loss and hyper- and hyponatremia. Unlike in the general population, kidney recipients are more susceptible to developing hyponatremia from volume overload, a serious condition that can cause death in the kidney recipients (Gore et al., 2006).

Fat distribution can influence body composition changes that affect cardiac structure and function, impacting outcomes and contributing to major complications in kidney transplant patients (Weiner et al., 2012). For example, increased fat distribution leads to decreased cardiac function, which can cause myocardial infarction. The cardiac 
structure is altered by large deposits of adipose tissue in the atrioventricular groove and right ventricular epicardium. These compositional changes influence aortic stiffness and can cause cardiac complications, including systolic blood pressure fluctuations and atherosclerosis. The latter is strongly influenced by cholesterol levels, which is important, because changes in total cholesterol and triglyceride levels in kidney transplant recipients could affect metabolism and increase the risk for CVD (Weiner et al., 2012).

\section{Donor Source, Gender, Race, and Posttransplant Weight Gain}

\section{Donor Source}

The prospective study by Moore and Gaber (1996) aimed to determine the relationship between weight gain and donor gender in 50 kidney allograft transplant recipients. The study participants included living related donor recipients $(\mathrm{N}=11)$ and cadaver donor recipients $(\mathrm{N}=39)$. The study examined the relationship between kidney function in diabetic and non-diabetic recipients who gained weight compared to nonweight gainers. The collection of data occurred at the time of transplant and 6 months posttransplant. As a result, $76 \%$ of the participants gained a mean average of $4 \mathrm{~kg}$, while the other 30\% either lost weight or did not gain weight. The women's average weight gain was $8 \mathrm{~kg}$, while the men's average weight gain was $7 \mathrm{~kg}$ (Moore \& Gaber, 1996). Moore and Gaber (1996) reported that Live renal donor allograft recipients had a weight gain of $6 \mathrm{~kg} \pm 2 \mathrm{~kg}$, which was higher than the cadaveric recipients $(p<.0001)$ and participants with diabetes gained $5 \mathrm{~kg}$ while nondiabetics gained $4 \mathrm{~kg}(p<.0001)$. No association was found between weight gain and kidney function $(p>.0001)$ (Moore \& Gaber, 1996).

\section{Gender and Race}

Some studies explored the difference between weight gain in African Americans and Whites. Between 1983 and 1999, a retrospective review examined the records of 506 kidney transplant recipients following kidney transplantation (Baum et al., 2002). The study found that African Americans gained significantly more weight than Whites (13.6 $\mathrm{kg}$ versus $9.1 \mathrm{~kg} ; p<0.05$ ) during the first year following transplantation (Baum et al., 2002). Similarily, 2 and 3 years following transplant surgery African-Americans continued to gain more weight than Whites (16.2 kg and $16.4 \mathrm{~kg}$, versus $11.5 \mathrm{~kg}$ and 11.1 $\mathrm{kg}$, respectively; $p<0.05$; Baum et al., 2002). In addition, Clunk, Lin, and Curtis (2001) found a significantly higher weight gain in African Americans compared to Whites, as did Gallagher et al. (2000a) in a retrospective review of 974 kidney recipients. In another study Gallagher et al. (2000b) confirmed weight gain after kidney transplantation is common, with an average weight gain of $10.3 \mathrm{~kg}$. The study also found that over $87 \%$ of the weight gainers averaged a minimum of $2 \mathrm{~kg}$ over 12 months. 
Cashion et al. (2007) conducted a more comprehensive retrospective analysis of weight changes in 171 individuals one year following kidney transplant surgery from January 1998 to January 2002. The recipients were women and men, and African American and White (Cashion et al., 2007). Study outcomes included fasting glucose, triglycerides, creatinine levels, and BMI (Cashion et al., 2007). Descriptive analysis found that all 171 kidney transplant recipients had a significant increase in mean weight $(6.2 \mathrm{~kg} \pm 10.7 \mathrm{~kg} ; p<0.05)$ and BMI $(2.1 \mathrm{~kg} \pm 3.8 \mathrm{~kg} ; p<0.05)$ one year following kidney transplantation (Cashion et al., 2007). In addition, most of the African Americans $(30.5 \mathrm{~kg})$ were more obese than Whites $(29.5 \mathrm{~kg})$, and more women $(31.4 \mathrm{~kg})$ were obese than men $(29.2 \mathrm{~kg})$, regardless of ethnicity and gender (Cashion et al., 2007). The study also found higher levels of triglyceride in the obese compared to nonobese transplant recipients (Cashion et al., 2007).

Collectively, these studies confirm weight gain occurs following kidney transplantation with significant gain presented one year following kidney transplantation, with a particular difference among population specificity. For example, women had a greater weight gain than men, and African Americans had a greater weight gain than Whites. The seemingly predictable weight gain and obese status following kidney transplantation could possibly threaten the viability of the new kidney, as well as, the recipient's life, and even more so for African American recipients, who also have a higher incidence of hypertension.

\section{Other Factors Contributing to Excess Weight Gain in the Kidney Transplant Population}

There is substantial literature that suggests weight gain following kidney transplantation is a well-known occurrence. Attempts to explain this phenomenon have been attributed to several factors including immunosuppression medications (de Oliveira et al., 2014; McPartland \& Pomposelli, 2007; Ryan et al., 2014) increased caloric intake due to history of reduced dietary restrictions (Bloodworth, Ward, Relyea, \& Cashion, 2014; Cupples et al., 2012; de Oliveira et al., 2014; Ryan et al., 2014), decreased level of physical activities following transplant surgery (Cupples et al., 2012; de Oliveira et al., 2014; Ryan et al., 2014; Sanchez et al., 2007), and consequences of concomitant chronic diseases (Ryan et al., 2014).

\section{Immunosuppression Medications}

Weight gain often associated with immunosuppression medications (McPartland \& Pomposelli, 2007) can increase rejection and decrease the viability of kidney function (Allison, 2016; Bamoulid et al., 2016; Saemann \& Sunder-Plassmann, 2008; Snowsill et al., 2017). Other complications associated with immunosuppression medications and weight gain include new onset of HTN, hyperglycemia, Type II diabetes, CVD, and hyperlipidemia (McPartland \& Pomposelli, 2007). According to Aksoy (2016), there is an association between weight gain and posttransplant steroid therapy use. This study 
found, after 36 months, $9 \%$ of kidney recipients experienced weight gain due to increased appetite. The use of immunosuppressive medications, such as steroid therapy, may cause hypernatremia and fluid disturbance, which lead to other health-related complications (Calò et al., 2017).

A retrospective study investigated the association between weight gain and impact of immunosuppressive therapy, without steroids, in 203 kidney transplant recipients from January 2005 to December 2009 (de Oliveira et al., 2014). The average weight gain found was approximately 15 pounds, after first months, and $9 \%$ of the participants gained weight after 36 months, post-kidney transplantation (de Oliveira et al., 2014). de Oliveira et al. (2014) suggested a negative impact of steroid therapy on weight gain following kidney transplantation; instead the study reported significant weight gain was associated with creatinine levels in young female recipients and donors.

\section{Dietary Intake}

Following kidney transplant, recipients often experience weight gain that is attributed to increased appetite and an increased food consumption. Some studies have shown immunosuppression medications and steriod therapy increased the recipients' appetites, thus increased body weight and BMI (Elster et al., 2008). Bloodworth et al. (2014) found significant weight gain after kidney transplantation, with an average weight gain of $4.2 \mathrm{~kg}$ in a year. The study reported an increase in BMI $(p<.05)$ for participants $(n=229)$ that lived within 1 mile of a grocery store near the kidney recipients were asssociated with an increase in BMI $(p<0.05)$; however, fast food restaurants and convenience stores did not significantly lead to BMI changes.

\section{Physical Activity}

Physical activity plays a role in reducing weight gain and obesity, and in improving overall health after transplant, although few transplant recipients engage in physical activity (Dontje et al., 2014; Sanchez et al., 2007). O'Brien and Hathaway (2016) reported physical activity is essential following transplantation; however, they found no specific standards or recommendations for increasing posttransplant physical activity in the literature. Nevertheless, guidelines in performing physical activity should be compatible with the kidney recipient's cardiac tolerance and physical ability (Bellizzi, Cupisti, Capitanini, Calella, \& D'Alessandro, 2014). As health care providers begin to encourage and prescribe activity regimens for transplant recipients, it will be critical to select methods, like DXA scans, which have the ability to assess body components such as visceral fat tissue. 


\section{Measurement of Body Fat and Weight Gain}

In general, there are several measures available to monitor body weight and body composition components. The anthropometric measurements assess the ratio of size and proportion of the body's composition such as BMI and WC. These measurements are advantageous because they are widely available, rapid, safe, and inexpensive, making them ideal for clinical and hospital settings. Anthropometric measures do not directly measure body fat and therefore lack the ability to assess body fat and predict new onset and progression of diseases as well as other health complications found in kidney recipients.

In kidney recipients, DXA provides indirect measures of regional and whole-body composition. DXA measures masses of soft tissue and fat and lean tissues. Unlike anthropometric measures, DXA assesses visceral fat, whole body fat, subcutaneous fat, and lean mass and is useful to predict health risk in kidney recipients. In addition, DXA provides a precise whole-body measurement and can explain variations in predicting outcomes post-kidney transplants (Heyward \& Wagner, 2004).

The monitoring of weight gain and subsequent obesity following transplant surgery is essential in order to assess and evaluate new onset and progression of concurrent chronic conditions. The more precise measurement of weight gain and obesity requires assessment of body composition (Duren et al., 2008; Heyward \& Wagner, 2004). One option for measurement is called the direct method. A direct method refers to body composition methods that provide validated estimates of \%BF, FFM, muscle, bone density ( $\mathrm{Db}$ ), hydration, or other body components, while indirect methods refer to the methods of measuring body composition that estimate \%BF, FFM, muscle, Db, hydration, or other body components (Heyward \& Wagner, 2004). Regardless of the underlying cause, the need exists for accurate measures that reflect not just weight or BMI, but more specifically the distribution of body fat in areas most associated with weight-related morbidities. Such measures could be used not only to identify individuals who are at high risk but also more accurately monitor efforts to reduce fat mass.

\section{Body Composition Methods}

Early body composition research developed some important concepts; however, the methods were not practical nor precise for clinical settings due to the environmental conditions required for assessment. Later, an anthropometric model evolved, to estimate total body muscle mass which was an important contribution to body composition research. Today, many clinicians use simple and practical methods to measure FM and FFM with different measurement methods such as the two-compartment models and hydrodensitometry, air displacement plethysmography (ADP), anthropometric, or BIA three-and four compartment models, and multicompartment models. This section will discuss anthropometric methods and regional and whole-body composition measures, and the underlying assumptions of each method, accuracy, precision, reliability and validity, and population specificity measures and methods. 


\section{Anthropometric Measurement}

According to Duren et al. (2008), anthropometric body composition measurements involves several parameters relevant to measures such as area; body volume; body weight; lengths at the knee height, arm span, and stature; skinfold thickness; breadths of the elbow, bi-iliac, ankle, wrist, and biacromial; and bodily circumference at the head, trunk, waist, hip, calf, wrist, arm, and chest. These anthropometric variables are useful in the predictive equations in the determination of quantitative techniques used to measure an individual's body fat composition. For example, estimation of body composition measurements from skinfold thickness measurement is primarily based on the large proportion of total body fat that is located underneath the skin (Heyward \& Wagner, 2004). Therefore, by obtaining measures from skinfold thickness and using a method such as underwater weighing, the percentage of body fat could be estimated in a healthy population. Unfortunately, anthropometric measures are limited when used as a single measure (Heymsfield et al., 2005).

Common anthropometric measures assess total and regional body composition. BMI, WHR, and WC are relatively simple and inexpensive methods to assess body composition (Vazquez, Duval, Jacobs Jr, \& Silventoinen, 2007). These anthropometric measures are useful and widely used to assess body weight and several health-related risk factors associated with excess adiposity (Heymsfield, Peterson, Thomas, Heo, \& Schuna, 2016). However, anthropometric methods are not useful when tissue-based measurements such as VAT compartment performed by DXA and CT scans are needed to capture the association between adiposity and health-related conditions (Cordeiro et al., 2013; Vatanparast et al., 2009).

\section{Body Mass Index}

BMI, a ratio of weight $(\mathrm{kg})$ and height ${ }^{2}$, is an attribute of obesity. BMI guidelines assume there is an association between body fat and body mass, but there is not. Furthermore, BMI lacks the ability to calculate an individual's body composition and assess body fat. Since body fat is known as a primary physiological risk factor for morbidity and mortality (Gallagher et al., 2000b; Josse, Azizian, French, Kramer, \& Phillips, 2011), BMI is not a good measure to predict cardiac mortality (Josse et al., 2011) and its utility as a measure is limited in the general as well as the kidney transplant population.

According to Heymsfield et al. (2016), BMI is an acceptable measure for estimating total body fat and assessing adiposity. BMI measures are a problem for two reasons. First, they lack the ability to estimate body fat among different ages, genders, ethnicity groups, and athletic build (Schoeller et al., 2005), which are factors that affect the relationship between BMI and \%BF. Secondly, BMI measures often inaccurately misclassify individuals as underweight, overweight, or obese because of their age, gender, or ethnicity (Carpenter et al., 2013; Daniels, 2009; Heyward \& Wagner, 2004). 
For example, Heymsfield et al. (2016) found that the relationship between BMI and adiposity differed across race and ethnic groups, specifically among Whites, AfricanAmericans, and Mexican-Americans. This finding raises questions about the suitability of BMI use in different race and ethnic groups.

\section{Waist-to-Hip Ratio}

Waist-to-hip ratio (WHR) is an indirect measure used for assessing body composition and determining risk factors associated with cardiovascular and metabolic diseases. Table 2-3 provides the waist-to-hip circumference ratio norms for men and women. The WHR measure is a simple calcualtion of WC in centimeters divided by hip circumference in centimeters (Heyward \& Wagner, 2004; Simmons, 2001). According to (WHO), the cutoff point for at-risk metabolic complications for men is WHR $\geq 0.90 \mathrm{~cm}$ and WHR $\geq 0.85 \mathrm{~cm}$ for women. (Alberti, Zimmet, \& Shaw, 2006). However, the International Diabetes Federation recommended a different set of cutoff points for different ethnic groups: for Europids men the WHR $\geq 94 \mathrm{~cm}$ and WHR $>80 \mathrm{~cm}$ for women. Meanwhile, the recommendations for South Asians, Chinese, and Japanese men suggest WHR $>90 \mathrm{~cm}$ and WHR $>80 \mathrm{~cm}$ for women (Alberti et al., 2006).

The distribution of upper body fat called the android and lower body fat called gynoid typically is different in men and women. The upper body fat or central obesity is more common in men, while lower body fat appears more in women deposited on the hips and thighs. However, if a person is obese, they are often categorized into either group. The location of body fat is important when using WHR for examining excess weight and disease risk, especially in different genders and ethnic groups. Although WHR is a useful anthropometric measure for central adiposity and visceral fat, there are limitations between gender and ethnicity. One study found an association between increase in WHR and postmenopausal women (Donato, Fuchs, Oppermann, Bastos, \& Spritzer, 2006), because women experience a menopause fat distribution pattern that is similar to men during menopause. Murray (2006) found an association between increased WHR and myocardial infarction, for women at 0.83 and for men at 0.9 . Asians, typically, have a strong association between increased WHR and cardiac mortality (Josse et al. (2011). This is due to the waist circumference association with visceral fat. However, the WHR measure is limited in the kidney transplant population because the hip circumference is subcutaneous fat deposition and the waist circumference is subcutaneous and visceral fat.

\section{Air Displacement Plethysmography}

ADP is used to estimate body volume and does not provide measurements of the regions. It is a less expensive validated and accurate method to measure $\% \mathrm{BF}$ in obese and extremely obese kidney transplant patients compared to DXA (Heymsfield, 2005). ADP makes assumptions regarding other tissue density to estimate \%BF. ADP measures 
Table 2-3. Waist-to-Hip Circumference Ratio Norms for Men and Women

\begin{tabular}{llllcc}
\hline \multirow{3}{*}{ Gender } & Age & Low & Moderate & High & Very High \\
\hline \multirow{4}{*}{ Men } & $20-29$ & $<0.83$ & $0.82-0.88$ & $0.89-0.94$ & $<0.94$ \\
& $30-39$ & $<0.84$ & $0.84-0.91$ & $0.92-0.96$ & $<0.96$ \\
& $40-49$ & $<0.88$ & $0.88-0.95$ & $0.96-1.00$ & $<1.00$ \\
& $50-59$ & $<0.90$ & $0.90-0.96$ & $0.97-1.02$ & $<1.02$ \\
Women & $60-69$ & $<0.91$ & $0.91-0.98$ & $0.99-1.03$ & $<1.03$ \\
& $20-29$ & $<0.71$ & $0.71-0.77$ & $0.78-0.82$ & $<0.82$ \\
& $30-39$ & $<0.72$ & $0.72-0.78$ & $0.79-0.84$ & $<0.84$ \\
& $40-49$ & $<0.73$ & $0.73-0.79$ & $0.80-0.87$ & $<0.87$ \\
& $50-59$ & $<0.74$ & $0.74-0.81$ & $0.82-0.88$ & $<0.88$ \\
& $60-69$ & $<0.76$ & $0.76-0.83$ & 0.840 .90 & $<0.90$ \\
\hline
\end{tabular}

Note. Reprinted with permission from author Dale R. Wagner 9-16-18. Originally published in "Applied body composition assessment", by Heyward, V. H., \& Wagner, D. (2004), p. 78; out of print. 
are derived from 3-C compartment consisting of fat mass, lean body mass, and bone mass. These measures should be used with caution in the elderly and children because of the alteration in the density of FFM (Baumgartner et al., 1991).

\section{Dual-Energy X-Ray Absorptiometry}

DXA is one of the most popular methods for measuring whole body and regional body. DXA is able to discriminate between soft tissue mass, total body adipose, bone mineral, and bone mineral density. DXA remains a widely used indirect method for estimation of total body mineral density (TBMD), total body mineral (Mo), bone mineralfree lean tissue mass (LTM), fat mass (FM), fat-free mass (FFM=LTM=BMC), and soft tissue mass (STM=LTM+FM) compositional changes in human body composition research (Dordevic et al., 2018; Heyward \& Wagner, 2004; Ng et al., 2018). DXA provides a more precise and accurate measurement of estimating $\% \mathrm{BF}$ compared to anthropometric measures, because it can assess regional body composition at the tissue level (Heyward \& Wagner, 2004; Teixeira et al., 2001; Vatanparast et al., 2009), especially in men (Ball, Altena, \& Swan, 2004).

While DXA was not originally developed to measure body fat, it can be useful in this regard because the scans can be focused on a single area. This can be employed to measure visceral fat, which is a critical factor for renal transplant patients. The scans used can also be whole-body to measure overall body fat percentage.

Additional advantages include low exposure to radiation and no requirements for restriction prior to testing regarding eating, drinking, or exercise for renal recipients. DXA can distinguish VAT, a marker for increased risk for Type 2 diabetes and cardiovascular diseases (Latt, Maestu, \& Jurimae, 2018; Ng et al., 2018) from other tissues.. DXA also provides precise and accurate estimates of body composition such as percentage of body fat $(\% \mathrm{BF})$ from three-body compartments $(3-\mathrm{C})$ that consist of fat mass (FM), lean body mass, and bone mass (Table 2-4).

These whole-body and regional body measurements provided by DXA are based on a 3-C model that is part of two equations (soft tissue + bone and lean tissue + fat tissue). Assessment of regional body areas such as legs, arms, and trunk are advantageous in kidney transplant recipients to estimate fat, lean mass, and total regional bone mineral density (BMD; Heymsfield, 2005). Other estimates of soft-tissue composition such as $\mathrm{FM}$ and lean tissue mass are used to determine percentage of body fat $(\% \mathrm{BF})$ and fat-free mass (FFM). It is the estimate of soft tissue composition that contributes to the major assumptions of DXA methods and limitations of DXA (Heyward \& Wagner, 2004).

The DXA method has several limitations. One limitation is that it does not offer high resolution of soft tissue imaging; therefore, it cannot accurately assess soft tissue composition in the presence of large bone areas. DXA only directly measures two tissue 
Table 2-4. Bioelectrical Impedance Analysis Approaches and Uses

\begin{tabular}{lll}
\hline Approach & Model & \multicolumn{1}{c}{ Recommended Use } \\
\hline $\begin{array}{l}\text { Traditional BIA } \\
\text { (whole body } \\
\text { tetrapolar, single } \\
\text { frequency) }\end{array}$ & Series & $\begin{array}{l}\text { To estimate TBW and FFM in healthy clients with } \\
\text { normal hydration status and normal fluid } \\
\text { distribution }\end{array}$ \\
Segmental BIA & Parallel & To estimate ICW and BCM \\
& Series & $\begin{array}{l}\text { To measure fluid distribution or regional fluid } \\
\text { accumulation in clinical populations }\end{array}$ \\
$\begin{array}{l}\text { Multifrequency BIA } \\
\text { Cole }\end{array}$ & $\begin{array}{l}\text { To measure regional or segmental ICW } \\
\text { changes in the ECW/BCM and ECW/TBW ratios in } \\
\text { clinical populations }\end{array}$ \\
$\begin{array}{l}\text { Upper-body (hand-to- } \\
\text { hand) BIA and lower- } \\
\text { body (leg-to-leg) BIA }\end{array}$ & NA & $\begin{array}{l}\text { To estimate \%BF in healthy clients with normal } \\
\text { hydration status and normal fluid distribution }\end{array}$ \\
\hline
\end{tabular}

Note: $\mathrm{BCM}=$ body cell mas; FFM = fat-free mass; $\mathrm{ECW}=$ extracellular water; ICW = intracellular water; TBW $=$ total body water; NA $=$ not applicable (these analyzers are based on series model but do not provide impedance or resistance data).

Note. Reprinted with permission from author Dale R. Wagner 9-16-18. Originally published in Applied body composition assessment", by Heyward, V. H., \& Wagner, D. (2004), p. 93; out of print. 
compartments (i.e., FM and FFM); therefore, soft tissue measurements such as fat and lean tissue mass can only be measured in anatomical locations without bone. DXA measures cannot distinguish between the components of abdominal adipose tissue, like VAT and subcutaneous adipose tissue (SAT). The accuracy of estimates of body composition components measures by DXA are determined by the manufacturers' software which accounts for the inconsistencies in measurements (Heymsfield et al., 1997; Heyward \& Wagner, 2004).

Other limitations of DXA include in adults and an older kidney transplant population, especially when the underlying assumed value is not met, the validity of the results are compromised and the $\% \mathrm{BF}$ is either over- or underestimated (Heyward \& Wagner, 2004, p. 124). Variables that influence measurement errors include age, exercise, and diet. For example, age affects the assumed value of fat-free mass body density (FFBd), which changes the FFM value. FFBd value in men and women $>65$ years old ranges from 1.093 and $1.099 \mathrm{~g} / \mathrm{cc}$, compared to $<65$ years old, which assumes the value of $1.1000 \mathrm{~g} / \mathrm{cc}$ (Heyward \& Wagner, 2004, p. 124). This is also true for relative hydration of FFM in older women (73.6-75.6\% FFM) and older men (72.4-74.4\% FFM), which affects body composition in the kidney transplant population. This variable contributes to the limitation of DXA and typically underestimates $\% \mathrm{BF}$ in an older population (Heyward \& Wagner, 2004, p. 133). Need to look quotes up out of book

The validity of DXA is challenged for several reasons. The validity of DXA is difficult to evaluate because the assumptions used to derive body composition estimates of soft tissue body composition are considered proprietary by DXA manufacturers (i.e., Lunar, Hologic, and Norland). The DXA method has body size restrictions based on the width and length of the equipment table. The ability to perform DXA scan in a morbidly obese patient poses a challenge because of a maximum weight restriction.

\section{Bioelectrical Impedance Analysis}

Bioelectrical impedance analysis (BIA) is a rapid, noninvasive method used for measuring body composition. Bioelectrical impedance indirectly estimates the volume of the body's TBW or FFM when a low-level electrical current is carried by water and fluids through the body. Therefore, there are certain assumptions and principles surrounding this method. However, this section will describe the two principles of BIA (Dehghan \& Merchant, 2008; Ng et al., 2018). First, the principle of BIA is based on the biological characteristic of tissues acting as conductors or insulators, and the direction of the electrical current transmitting through the path of least resistance. For example, FFM contains $\sim 73 \%$ of water and electrolytes, which makes it a better conductor than fat, which is considered anhydrous with a poor electrical conduction (Heyward \& Wagner, 2004). This method uses the two factors that human body is composed of highly conductive tissue referred to as lean body mass and less conductive tissue with an insulator such as body fat and measured impedance reflects the ratio between conductive tissue and nonconductive tissue, which is why it is called bioelectrical impedance analysis. Secondly, impedance is a function of resistance and reactance. Resistance is a 
measure of pure opposition to current flow through the body, and the reactance is the opposition to current flow caused by voltage storage produced by the cell membrane (Gonzalez, Orlandi, Santos, \& Barros, 2018). Clients must meet certain conditions prior to BIA:

- No eating or drinking within 4 hours of the test.

- No exercise within 12 hours of the test.

- Client should urinate within 30 minutes of the test.

- No alcohol consumption within 48 hours of the test.

- No diuretic medications within 7 days of the test.

- No testing of female clients who perceive they are retaining water during that stage of their menstrual cycle.*

The advantage of using BIA is that it is a quick, noninvasive, and inexpensive method that can be used in the clinical or field setting to estimate body composition with obese patients, and it does not require a highly skilled technician (Heyward \& Wagner, 2004). The BIA method is recommended for assessing percent of body fat in a pre- and posttransplantation heart, liver, and lung population (Heyward \& Wagner, 2004). A disadvantage in using BIA is that it may lead to measurement error. To avoid measurement errors, the test individual must adhere to the BIA pretesting client guidelines just shown.

\section{Computed Tomography}

Computed tomography $(\mathrm{CT})$ uses radiation to create an image to assess $\mathrm{BC}$ at the tissue and organ level (Heyward \& Wagner, 2004; Heymsfield et al., 2005). CT accurately measures body composition, such as bone, adipose, and lean tissues (Heyward $\&$ Wagner, 2004). There are two advantages of CT. First, it is more reliable than MRI (Heyward \& Wagner, 2004). Second, CT can measure large body sizes (Duren et al., 2008). However, there are several disadvantages, including the cost and exposure to radiation, requirement of a skilled technician (Heyward \& Wagner, 2004), the need for special software, and only regional not whole body imaging of body composition (Duren et al., 2008).

\section{Magnetic Resonance Imaging}

Magnetic resonance imaging (MRI) measures body composition at the tissue level (Heyward \& Wagner, 2004; West et al., 2018). MRI produces a computer-generated

* Reprinted with permission from author Dale R. Wagner 9-16-18. Originally published in "Applied body composition assessment", by Heyward, V. H., \& Wagner, D. (2004), p. 94; out of print. 
image using a radio frequency signal from hydrogen nuclei. The hydrogen nuclei act like a little magnet (Heyward \& Wagner, 2004). The advantage of MRI is that it can be used for full body scan in patients with normal weight or moderately overweight (Duren et al., 2008). The disadvantages of MRI are the same as for CT.

\section{The Challenge of Measuring Body Fat and Weight Gain}

Body composition measures are important components to monitor short-term and long-term impact in the general population and health outcomes. Weight gain and increased body fat cause enlarged adipocytes and increased VAT in the abdominal area, which could subsequently lead to diabetes or CVD (Pi-Sunyer, 2018). Obesity is a global epidemic (Despres, 2012; Ghoorah et al., 2016; Kovesdy et al., 2017) and risk factors for chronic diseases and poor health outcomes in transplant recipients. Several direct (e.g., human cadaver) and indirect BC methods (e.g., anthropometric method, BIA and DXA) can be used to measure body fat and weight gain and have been previously discussed. While these methods are used to measure body fat and weight gain in the general population, challenges exist with using indirect methods to measure body fat and weight gain in the general population and those with clinical conditions. Age, ethnicity/race, and clinical conditions impact the measurement of body fat and weight gain in the general population and/or kidney transplant population.

\section{Age}

Physiological changes occur with aging and significantly affect changes in body composition. During the aging process, water, mineral, and protein components of FFM are altered, which affects the FFMd (Heymsfield, Peterson, Thomas, Heo, \& Schuna, 2016; Heyward \& Wagner, 2004; Jura \& Kozack, 2016; Shaw et al., 2007). For example, as the body ages, excessive body weight in the older population versus the younger population is characterized by increased FM and may contribute to an increase in distribution of internal deposits of visceral fat, leading to medical conditions associated with abdominal fat such as diabetes. The presence of increased visceral fat is associated with a concurrent decrease of muscle mass (Kathryn, Shelley, Julia, \& Connie, 2016; Sharp, Andrew, Burchfiel, Violanti, \& Wactawski-Wendek, 2012).

Obesity in an older population is characterized by an increase in body weight, which results in an increase in deposits of visceral fat and abdominal fat in the body (Elia, 2001; Heyward \& Wagner, 2004; Jura \& Kozack, 2016). Increased deposits of visceral fat and abdominal fat in the body are subsequently accompanied by an increased risk of morbidity and mortality (Elia, 2001) from chronic diseases (Heyward \& Wagner, 2004; Jura \& Kozack, 2016). In addition, the physiological changes in the older population affect measurements of $\mathrm{BC}$. For this reason, problems exist with using anthropometric measurements such as SKF to measure BC in the older population, particularly because of decreased elasticity and skin hydration, as well as, the shrinkage 
in fat cells may cause an increase in the measurement for SAT and connective tissues (Guerra, Amaral, Marques, Mota, \& Restivo, 2010).

Snead, Birge, and Khort (1993) studied age-related differences in BC by hydrodensitometry (HD) and DXA to determine if \%BF was overestimated with HD in older male and female population because aging leads to a decrease in bone mineral content. The studied consisted of 113 women and 72 men between the ages of 21 to 81 (Snead, Birge, \& Khort, 1993). Snead et al. (1993) found that HD and DXA underestimated \%BF in the older population, with DXA significantly underestimating it by $4 \%$ to $5 \%$ (Snead et al., 1993).

Shaw et al. (2007) used DXA BC and anthropometric measurements to study aging in a population-based older cohort. Seven hundred and thirty adults between the ages of 50 to 79 who were men and women were measured with standardized measurements of BMI, WC, WHR, and DXA scan from March 2002 to January 2004 (Shaw et al., 2007). The DXA scan focused on four measurements: total body fat mass, $\%$ total body fat, $\%$ trunk fat, and lean body mass (Shaw et al., 2007). Three separate statistical analyses were performed on men and women. Linear regression was used to test the trends in BC with the separate age categories of men and women (Shaw et al., 2007). Partial correlations and Bland-Altman analysis were used to measure if a consensus occurred between DXA and anthropometric measurements (Shaw et al., 2007). Results showed lean body mass with DXA decreased significantly with the advancing age of the men and women $(p<0.05)$. In males, BMI and body weight decreased with advancing age $(p<0.01)$. In aging females, DXA showed an increase in fat, $\% \mathrm{BF}(p<$ $0.02), \%$ trunk fat $(p<0.05)$, and WHR $(p<0.05$; Shaw et al., 2007). No differences existed between DXA total body fat, WC, or hip circumference (Shaw et al., 2007). These results showed a high consensus existed between DXA measurements, BMI, and WC in the measurement of BC in the older cohort population. However, WHR was not consistent with DXA measurements of BC in the older cohort population. This study concluded there was an overall consensus between the BC measurement of DXA and anthropometric methods except WHR, which suggests that WHR is not a suitable BC measurement in the older population (Shaw et al., 2007).

\section{Ethnicity/Race}

Obesity differentially impacts certain ethnic and racial groups (Heyward \& Wagner, 2004; Richmond et al., 2015; Wang et al., 2017). A report from a national survey (2011-2012) in the United States showed Asians have the lowest percentage of obesity and Blacks have the highest (Wang et al., 2017). The survey specifically reported obesity rates for Asians were 10.8\%, Whites 32.6\%, Hispanics 42.5\%, and African Americans 47.8\% (Wang et al., 2017). Wen and Kowaleski-Jones (2012) corroborated results of the national survey reporting the highest rate of obesity was found in Hispanics and African Americans, with Asians and Whites experiencing lower rates. 
While the rate of obesity differs among the four ethnic groups, specific BC measurements (e.g., BMI and WHR) may not accurately estimate \%BF, resulting in misclassification of some individuals to high- or low-risk groups (Heyward \& Wagner, 2004). For example, in a study by Heyward and Wagner (2004), DXA classified $25 \%$ of American-Indians as obese, but HD and a three- body composition classified $78 \%$ of American-Indians as obese. The different estimation of obesity with the DXA, HD, and 3-body composition showed problems exist with using these three BC methods to classify obesity in American Indians. According to Heyward and Wagner (2004), ethnic differences exist across the groups with subcutaneous fat distribution from the trunk to the extremity and limb lengths to total height. These differences may make it difficult to estimate \%BF among the different ethnic groups accurately; therefore, a multicomponent model should be used as a reference method since 2-C models and DXA are the only models used to accurately estimate $\% \mathrm{BF}$ in the different ethnic groups (Heyward \& Wagner, 2004). Thus, standardized BC methods and equations must be developed to estimate $\% \mathrm{BF}$ accurately in different ethnic groups.

\section{Clinical Conditions}

Clinical conditions may make certain BC methods unsuitable for accurate assessment of $\% \mathrm{BF}$. It is important to acknowledge DXA measures and reference databases should not be used to diagnose disease or conditions, nor provide recommendations for treatment regimens. Instead, the presence or absence of certain diseases is a critical step for selecting accurate methods and measures in body composition. The clinical conditions discussed as exemplars are pregnancy and kidney failure and dialysis, although other examples exist such as trained athletes and muscular wasting or fluid retaining chronic conditions (Heyward \& Wagner, 2004).

\section{Pregnancy}

One anthropometric method used to safely assess body composition in pregnant women is SKF (Heymsfield et al., 2005). However, a study found that SKF overestimated \%BF in pregnant women. Robic et al. (2018) examined the best anthropometric methods to use in pregnant women to measure body fat and concluded that body height, body weight, SKF, and limb circumferences were the best measures. The three methods provided a wide range of prediction equations for estimating body fat in pregnant women.

\section{Kidney Failure and Dialysis}

Kidney failure results in metabolic waste accumulation in the body from a decline in kidney function. The treatment for kidney failure is dialysis. Dialysis removes metabolic waste and excess fluid from the body (Heyward \& Wagner, 2004), thereby influencing body composition, yet there is no standardized method to assess BC in dialysis patients. The methods used to assess $\mathrm{BC}$ have led to varying results, either

overestimating or underestimating \% $\mathrm{BF}$ in kidney failure and dialysis patients (Heyward 
\& Wagner, 2004). This suggests total body water is strongly associated with hypertension.

Cooper et al. (2000) studied 54 patients with end-stage kidney disease. Deuterium dilution technique was used to estimate TBW and compared to estimates from anthropometric methods, 58\% body weight, Watson equations, and BIA. FFM produced from anthropometric methods was also used to estimate TBW and compared with BIA estimates of TBW. Last, measurements of total body nitrogen (TBN; $p=0.04$ ) were linked with TBW estimates and BIA-derived resistance. The results showed the Watson equation significantly underestimated TBW $(p=0.01)$, and overestimated body weight. The BIA equation did not significantly differ from the gold standard methods used to estimate TBW ( $p=0.12$. Nevertheless, the agreement varied on the equation methods used to estimate TBW, but BIA obtained resistance accurately estimated TBW and other body composition components (Cooper et al., 2000).

Kang, Cho, Park, Yoon, and Do (2014) studied 41 Asian patients on maintenance peritoneal dialysis to determine the effect of peritoneal dialysate on BIA measurement of BC. Prior to multifrequency BIA measurement, dialysate was drained from the abdomen with the patients standing (D-) (Kang, Cho, Park, Yoon, \& Do, 2014)(Kang, Cho, Park, Yoon, \& Do, 2014). Dialysate was administered in the patients and measurement was taken and repeated (D+). Bland-Altman was used to analyze agreement and bias. Bias was D+ measurement and D- measurement (Kang et al., 2014). The presence of peritoneal dialysate increased ICW (D-: $20.33 \pm 3.72 \mathrm{~L}$; D+: $20.96 \pm 3.78 \mathrm{~L}$ ), ECQ (D$: 13.53 \pm 2.54 \mathrm{~L} ; \mathrm{D}+: 14.10 \pm 2.59 \mathrm{~L}$ ), and TBW (Kang et al., 204). The increase of ICW and ECW in the presence of peritoneal dialysate was significant $(p<0.001)$. In the presence of peritoneal dialysate, total and trunk edema indices were higher, but both extremities were not significantly different (Kang et al., 2014). Moreover, mineral content and FFM for total body and trunk were overestimated and body fat was underestimated (Kang et al., 2014). In the presence of peritoneal dialysate, trunk had a lower BIA measurement, but in both extremities, no changes were found in BIA measurements (Kang et al., 2014). Biases were found in soft lean mass (1.53 kg), FFM $(1.68 \mathrm{~kg})$, fat mass $(-1.71 \mathrm{~kg})$, and edema index $(0.003 \mathrm{~kg})$. The overall findings from the Kang et al. (2014) study showed in the presence of peritoneal dialysate agreements and biases exist with BIA measurements.

\section{Multicompartment Methods}

There are five distinct body composition levels: atomic, molecular, cellular, tissue-organ, and whole-body. Each level is divided into discrete components, except the whole-body level, which is divided into regions. First, the atomic level contains four major elements that make up $96 \%$ percent of body mass: oxygen, carbon, hydrogen, and nitrogen. These major elements are used by various models to estimate total body fat, body cell mass, and protein. In addition, seven other elements compile the atomic level, including calcium, phosphorous, potassium, sodium, sulfur, chlorine, and magnesium. On the other hand, there are six major components at molecular level: protein, bone 
mineral, lipid, water, carbohydrate, and soft tissue mineral. These components are used to develop complex models that consist of three to six components referred to as multicomponent models. The cellular level is compiled of three components: extracellular solids, extracellular fluids, and cells. At this level, the cells are further metabolized into fat and body cell mass (BCM) components. The fourth level of body composition is the tissue-organ level. The major components at the tissue-organ level include adipose tissue, skeletal muscle, visceral organs, bone, and other single organs such as brain, heart, liver, and spleen. The final body composition level is the whole-body level. This level is divided into three regions: appendage, trunk, and head. The regions at this level provide anthropometric measures such as skinfolds, length, and circumference, which are used in estimation of prediction equations.

According to Wang, Pierson Jr, and Heymsfield (1992), the two-compartment (2C) model was developed to standardize a basic method for assessing body fat, particularly in the absence of weights of measure components (Table 2-1). Twocomponent models divide the body into fat and fat-free body components. The limitation of the two-component and the DXA method (Withers et al., 1998) involves the assumption of constant hydration of water of $73 \%$ in FFM, sex, and body size, which is inaccurate. The three-component model (3-C) model divides the body into fat, water or mineral and residual components. The 3-C model precision is better for estimating body composition variation of body fat, and is preferred when compared to the 2-C reference methods, including hydrodesitometry (HD) and TBW. The HD method evolved into a 3-, 4-, and 6- model. This model is useful in DXA, BIA, and ADP devices.

The Siri's three-component (3-C) hydrodensitometry method was derived from the Behnke's two-component (2-C) method, which was composed of known and constant proportions of fat-free mass (FFM). The 3-C method is composed of fat, water, and residual components. The expansion of the 3-C method led to the estimation of three quantifiable measures that included body mass, body volume, and total body water (TBW). The inclusion of TBW reduced the errors associated with Behnke's 2-C method. Later, the 3-C method expanded to the four-component (4-C) method by adding a bone mineral content, which reduced possible measurement errors associated with bone mineral. These 4-C multicomponent methods quantify the following measures: body volume, TBW, body mass, and bone mineral. On the other hand, the six-component (6C) methods such as neutron activation method include fat, total body water, protein, bone mineral, soft tissue mineral, and glycogen, whereas the 6-C total body carbon method includes components at the molecular level such as fat, protein, and glycogen; it requires direct analysis of the chemical composition of the body in vivo.

The 3-C model is not recommended in the KTR population or any population with impaired kidney function and physiological compensation related to age or disease. Additionally, it is recommended that the 3-C (water) or the 4-C (water) should be used to obtain reference measures of body composition of older adults. The FFM is the model divided by its own water content and the additional solids, primarily protein and minerals. Three-component calculations combine fat, density of water, fat, and body solids to estimate body fat mass. An inaccurate estimate may occur if an individual has a 
significant decline in body protein mass, BMM, or when there is estimated density for solid compartments (Heyward \& Wagner, 2004).

Heyward and Wagner (2004) found that age, sex, and ethnic group body composition differed during body compositional changes at the molecular level: lipid metabolism, protein, nutrition area, water balance, bone and mineral, and homeostasis. Measurable properties at this level are DXA method, which are used to assess wholebody composition. Because adipose and fat are measured at different component levels, MRI and CT would not be a suitable reference for estimates of fat by another method such as DXA underwater weighing (UWW). Fat is at the molecular level and DXA lacks direct measures in the regional areas and can only provide estimates of percentage of body fat from soft tissue mass, fat mass, and lean body mass.

The multicompartment (4-C) model is a highly significant predictor of the relationship between age and ethnicity. For example, Asian populations have a high level of adipose, and an anthropometric method such as BMI could prolong the risk for cardiovascular disease. MRI would not be a suitable reference comparison for estimates of fat by another method that considers the variability of water or mineral content of the fat-free body, or both. They are generally thought to provide more accurate estimates of body fat than 2-component models, especially when one of the assumptions of the 2component model might be violated, such as constant hydration, which is not likely in the kidney transplant population (Heyward \& Wagner, 2004).

In addition, in vivo neutron activation analysis (NAA), the 4-C model, measures bone density and unknown quantity of mass for each body compartment. The unknown values are obtained from DXA measures, which have the ability to quantify bone mineral content or NAA that quantify the protein content. The indirect pathway to estimate FM from two additional methods, DXA and NAA, makes the 4-C UWW method cumbersome and time consuming for technicians and participants. However, the 4-C UWW and DXA are more commonly used than 4-C UWW and NAA (Heyward \& Wagner, 2004). UWW, which is also referred to as hydrostatic weighing, is considered the gold standard for measuring body composition from body density. There are several methodological issues that occur when comparing a person's underwater weight to their dry-land weight, which is the inversely proportional to body fat, including the following: subject position, residual volume, number of trials and selection criteria, alternative lung volumes, and head placement. The subjects' positions include sitting, kneeling, or prone, which generally depends on the size of the tank. However, measurement errors may occur if the subject is tall or has a large abdomen. In addition, UWW requires the subject to exhale completely under the water; therefore, potential measurement errors may occur if the subject lacks the ability to completely exhale underwater, thus reducing the accuracy and reliability of the measurements of the subjects. The technician's ability to accurately determine the repeated measurements of a subject is another possible measurement error (Heymsfield et al., 2005). UWW method is limited in specific populations including young children, disabled, elderly, sick, and other special groups. The complete submersion in water could cause complications and may not be possible in certain populations. 
The 4-C DXA component model is preferred for an aging population for estimating $\% \mathrm{BF}$. This model calculation assumes the bone mineral calcium and bone mineral in carbon and the measures are adjusted for variation in an older population due to the decline in bone and muscle components. If the 4-C is not available, the 2-C is an alternative method to improve measures for estimating $\% \mathrm{BF}$, by averaging the value of fat-free body density (FFBd). This adjustment for FFBd is critical in different ethnic and racial groups because of the variation in bone density mass (Heyward \& Wagner, 2004).

A 4-C molecular model should not be considered in kidney transplant recipients or any population with chronic disease who may experience fluid disturbance such those individuals with electrolyte imbalance. Transplant recipients often experience fluid disturbance and electrolyte imbalance, in which the 4-C components of fat, protein, bone mineral, and total body water (Heyward \& Wagner, 2004) can be used to derive a suitable predictive equation.

Studies have shown (Heyward \& Wagner, 2004) BIA equation accurately estimates changes in FFM and TBW in obese women on a low caloric diet. Several studies have shown that the BIA method is sensitive to detect body composition change relative to weight loss. On the other hand, BIA method FFM is not accurately reported if weight loss occurs. The BIA method is not useful if hydration is altered or there is a fluid disturbance. However, the NIR method was recommended in subjects with fluid disturbance such as a dialysis patient.

Studies have shown BIA measures derived from SKF are suitable for predicting percentage of body fat (Heyward \& Wagner, 2004). BIA estimate is suitable body composition in obese population for several reasons.

\section{Disease-Specific Population}

The BIA method is best for disease-specific populations. Predictive equations are useful in a population with metabolic syndrome, particularly in a disease-specific population with alteration in fat distribution and fluid disturbance. Two diseases, known as Type 2 diabetes or thyroid diseases, do not have a predictive equation. The BIA method or Segal fat-specific measure are the best measures and methods to use in obese and Type II diabetes populations. The Leiter Disease-Specific Predictive BIA equation is more preferred in a disease-specific population compared to the BIA method. Understanding the interaction between methods and measures could be a challenge in clinical practice when selecting suitable methods to improve outcomes. 


\section{Disease-Gender Population}

Heyward and Wagner (2004) used a disease-gender prediction equation to estimate percentage of fat in a cardiopulmonary population. The BIA method and Geneva predictive equation DXA measures are suitable for chronic obstructive pulmonary disease. This predictive equation is used in equipment called Xitron-4000 BIA. In some cases, the measure and method must factor in disease and gender. For example, the BIA - BIO-Z method is suitable for men and women with cystic fibrosis. On the other hand, the measure is not gender specific. Men with cystic fibrosis could use DXA measures and the Geneva predictive equation for more accurate assessment of $\% \mathrm{BF}$ compared to women using measures by Kotler.

Studies have shown (Heyward \& Wagner, 2004) BIA equation accurately estimates changes in FFM and TBW in obese women on a low caloric diet. Several studies have shown that the BIA method is sensitive to detect body composition change relative to weight loss, except when weight loss occurs rapidly. BIA method is not useful if hydration is altered or fluid disturbed. However, the NIR method was recommended in subjects with fluid disturbance such as a dialysis patient. Studies have shown BIA measures derived from SKF are suitable to predict percentage of body fat in an obese population but not in an extremely obese population (Heyward \& Wagner, 2004). Other recommendation UWW method included limited in specific populations including young children, disabled, elderly, sick, and other special groups. The complete submersion in water could cause complications and may not be possible in certain populations.

\section{Summary}

In summary, kidney transplantation is the preferred choice of treatment for patients with ESRD. After transplantation, kidney recipients experience short-term and long-term complications, such as weight gain. In the kidney transplant population, weight gain is a serious issue. Factors affecting weight gain in the kidney transplant population posttransplant are immunosuppression medications, increased caloric intake from reduced dietary restrictions, decreased level of physical activities, and consequences of concommittent chronic diseases. The monitoring of weight gain and subsequent obesity following transplant surgery is essential to assess and evaluate new onset and progression of concurrent chronic conditions. Although indirect methods, such as anthropometric method, BIA, and DXA, are commonly used methods to measure body fat and weight gain in the general and kidney transplant population, challenges exist with using indirect methods to measure body fat and weight gain with patients with certain biological and pathological conditions. 


\section{CHAPTER 3. METHODS}

There is little consensus on which method of measuring body composition is superior for identifying patients at increased risk for CVD. Therefore, the overall aim of this study was to compare associations of anthropometric measures versus those obtained by DXA scan with cardiac-related risk factors at 5-8 years following kidney transplantation. The specific aims addressed by this study were:

1. To determine the association between anthropometric measures and serum levels of glucose, lipids, creatinine, and blood pressure of kidney transplant recipients at 5-8 years posttransplant.

2. To determine the association between body composition measures and serum levels of glucose, lipids, and creatinine and blood pressure of kidney transplant recipients at 5-8 years posttransplant.

3. To determine if body composition measures by DXA obtained at the time of transplant (baseline) are associated with serum levels of glucose, lipids, and creatinine and blood pressure of kidney transplant recipients at 5-8 years posttransplant.

4. To determine if anthropometric measures obtained at baseline are associated with serum levels of glucose, lipids, and creatinine and blood pressure of kidney transplant recipients at 5-8 years posttransplant.

5. To determine the association between changes in anthropometric measures from baseline to 5-8 years posttransplant and serum levels of glucose, lipids, and creatinine and blood pressure in kidney transplant recipients at 5-8 years posttransplant.

6. To determine the association between changes in body composition measures from baseline to 5-8 years posttransplant and serum levels of glucose, lipids, creatinine, and blood pressure at 5-8 years posttransplant.

The remainder of this chapter describes the methodology used to address the above research aims, including the research design, setting and sample, instrumentation, method and subjects, data analysis, statistical assumptions, and a summary.

\section{Research Design}

A quantitative, descriptive follow-up study was conducted that included data acquired during a parent study conducted between 2008 and 2011 (Cashion et al., 2014), which examined genetic, environmental, and lifestyle factors contributing to post-kidney transplant obesity. The parent study data were collected at the time of transplantation and 
served as baseline measures. Additional data were collected for this follow-up study, which took place 5 years following completion of the parent study.

\section{Setting and Sample}

\section{Setting}

The parent study was conducted in a large sized midsouth university with an affiliated transplant institute that has been performing kidney transplants for over 40 years. Demographic characteristics of the 120 to 130 individuals receiving kidney transplants each year in this institute was reflective of the regional population. The immediate urban area surrounding the transplant institute was predominately of African Americans descent, many of whom resided in lower socioeconomic communities. The transplant institute also provided services to individuals located in rural communities and other urban cities in the region and across the country. Data from 2016 to the present indicated demographic characteristics of kidney transplant recipients at this center had remained relatively stable with 27.7 to $28.4 \%$ being Whites, and 69.1 to $69.6 \%$ African Americans; 1.6 to $1.9 \%$ Hispanics, 1.6 to $1.0 \%$ Asians; 2.8 to $65.6 \%$ were males and 47.1 to $34.3 \%$ were females; 12.1 to $10.7 \%$ were $8-34$ years, 34.9 to $28.4 \%$ were $5-49$ years, 44.4 to $46 \%$ were $0-64$ years, and 11.3 to $14.7 \%$ were $65+$. This study, although reflective of the population served, had a higher proportion of African American patients receiving transplants than do other high-volume centers.

\section{Sample}

The parent study (Cashion et al., 2014) included 96 post-kidney transplant recipients who were 18 years or older; $42.71 \%$ were females and $57.9 \%$ males. By ethnicity, $53.12 \%$ were Non-Hispanic and 2.08\% Hispanic. By race, $65 \%$ were African Americans, 31\% were Whites, and 4\% were other. Of the 96 participants in the parent study, 45 patients received DXA scans at the transplant baseline time-point and 54 received a scan at 1 year. Because additional study participants received DXA scans at the 1 year following posttransplant, all 96 participants of the parent study were screened for eligibility for the follow-up study.

\section{Inclusion Criteria}

Participants in the parent study who had a DXA during the peritransplant and/or 1 year after kidney posttransplant were included in this study. 


\section{Exclusion Criteria}

- Current hospitalization.

- Loss of the transplanted graft.

- Receipt of steroid therapy prior to the time of transplantation to control for the effect of steroid use on baseline weight.

\section{Instrumentation}

In the current study, empirical data were obtained using a) anthropometric assessment, (b) body composition assessment, and (c) physiological assessment.

\section{Anthropometric Assessment}

Anthropometric assessments included measurement of Body Mass Index (BMI), Body Weight (BW), and Waist Circumference (WC). These measures are defined as follows:

- Body Mass Index (BMI) measures weight and height by calculating weight in kilogram $(\mathrm{kg})$ divided in height in meter squared $\left(\mathrm{m}^{2}\right)$ (Caballero, 2014).

- Body Weight (BW) measures human body total mass in kilograms or pounds.

- Waist Circumference (WC) measures the central abdominal area.

Height, weight, and waist measurements were obtained from each participant by the principal investigator. Each participant was required to remove shoes and bulky outer garments before height and weight were measured. Height was obtained using a wallmounted scale and measured to the nearest centimeter. A calibrated balance scale was used to assess each participant's weight in kilograms. BMI was calculated using each participant's weight in kilograms divided by the height in meters squared (Antillon \& Towfighi, 2011; Beckmann et al., 2015; Caballero, 2014; Ghoorah et al., 2016; WHO, 2018). A tape measure was used to measure each participant's WC by holding the end of the tape measure at the participant's navel and then bringing it around the participant's waist and back to the front.

\section{Body Composition Assessment}

The DXA scan was performed by a DXA-certified research nurse to measure the distribution of fat and lean mass in various compartments of the body. Specifically, the android and gynoid ratio quantifies the adipose tissues that accumulate in the abdominal 
region and around the hips, total body mass percentage (total body mass minus bone and fat), and the amount of fat tissue located deep in the abdomen and around the internal organs (Heyward \& Wagner, 2004), as follows:

- Android/Gynoid Ratio: Android fat is the amount of fat between the bottom of an individual's head and the top of the iliac crest in the lowest $20 \%$ region. Gynoid fat is the amount of fat located downward from the android region and from the top of the greater trochanter. It is derived from $\%$ fat in the android region divided by $\%$ fat in the gynoid region.

- Android Fat Mass (AFM) is the amount of adipose tissue in the abdominal area and below the individual's head. It is derived from the fat tissue in the android region (grams).

- Gynoid Fat Mass (GFM) is the amount of fat in the lower portion of an individual's body around the hip area. It is derived from the fat tissue in the gynoid region (grams).

- Total Fat Mass (TFM) is the fat mass in grams (android + gynoid regions). It is derived from the standard deviation value.

- Lean Mass (LM) is the total body mass less the bones and fat in an individual's body total lean mass (android + gynoid area). It is derived from the lean standard deviation value.

- Percentage of Body Fat (\%BF) is an individual's total amount of fat divided by total body weight percent fat (android + gynoid regions).

- Visceral Adipose Tissue (VAT) is the amount of fat tissue surrounding an individual's organs that impacts a wide variety of clinical risks (Bergman et al., 2006). VAT is a valid predictor of cardiometabolic risk factors and the study found that DXA clinical thresholds were validated in White men $(154 \mathrm{~cm} 2)$ and women $(143 \mathrm{~cm} 2)$ compared to African American men $(101 \mathrm{~cm} 2)$ and women $(114 \mathrm{~cm} 2)$. VAT is derived from fat tissue in the largest visceral fat region in grams (Heyward \& Wagner, 2004).

\section{Physiological Assessment}

The blood collection for glucose, total cholesterol, LDL-cholesterol, HDLcholesterol, and triglycerides was performed in the clinical research center by a registered nurse at no cost to participants. Forty cubic centimeters (about 3 tablespoon) was collected from each participant in a BD vacutainer SST tube for serum determination in ethylenediaminetetraacetic acid (EDTA) tubes for anticoagulation. Each tube was inverted after the blood samples were collected to prevent clotting. The blood samples 
were stored on ice for no more than two hours before blood sample centrifugation. The plasma and serum were separated into cryotubes and stored at $-80^{\circ} \mathrm{C}$ until processed.

Table 3-1 summarizes the normal value of the blood samples in the physiological assessment.

\section{Method and Subjects}

Prior to research, approval from the university Institutional Review Board (IRB) was obtained (IRB Approval Supplement) as well as approval of the parent study by the principal investigator. Potential study participants were identified from the parent study database; those who met inclusion criteria $(n=96)$ were contacted by phone, the followup study described, and the opportunity for them to participate in the study offered. Twenty-one out of the 96 transplant patients were excluded from the study due to loss of graft functions or death $(n=45)$, two participants declined, and those expressing interest in the study were scheduled to meet with the Principal Investigator at the General Clinical Research Center (GCRC). Appointments were coordinated with an upcoming routine clinic appointment, or another time at the potential study participant's convenience. The GCRC was located in the same building, just a few floors above the transplant clinic; research staff met potential participants in the transplant clinic and escorted them to the GCRC, if needed.

After greeting the potential study participant, the research staff described the study again in detail and reviewed the consent page by page. Questions were solicited, and the potential participants understood their participation in the study was voluntary and that no additional appointments were required. Each potential participant was assured they had the right to opt out of the study at any time and that such a decision would not affect their current or future medical treatment. Additionally, each person was informed that there would be no cost to them for the study procedures, including the DXA scan and blood studies, and that the results of these tests would be shared with them per request.

After the 45 participants signed the participant-informed consent (Participant Informed Consent Supplement), health records were reviewed as electronic medical records. The data retrieved from the parent study participants were laboratory values, BP, height, weight, and previous DXA scans. If a participant was a female of childbearing age, a urinary pregnancy test was performed prior to the scan.

During the single clinical visit, 40 cubic centimeters (about 3 tablespoons) of blood was drawn from each participant followed by a Whole-Body DXA scan using a DXA scanner die body composition estimate (Hologic QDR 4500A, software version 4.5.2.1, Bedford, MA). DXA equipment has set values for the fat standard deviation value, which is 68 , and the standard deviation value for lean tissue, which is always -10 . The calibration for this software estimate was the \%fat 3-4\% higher than the Classic software and underestimates that FM compared to the criterion methods in adults (Schoeller et al., 2005). 
Table 3-1 Normal Value of the Blood Sample

\begin{tabular}{lc}
\hline Blood Sample & Normal Value in mg/dl \\
\hline Glucose & $65-100 \mathrm{mg} / \mathrm{dl}$ \\
Hemoglobin A1C & $4.6-5.6 \%$ \\
Total Cholesterol & $<200 \mathrm{mg} / \mathrm{dl}$ \\
LDL-Cholesterol & $<100 \mathrm{mg} / \mathrm{dl}$ \\
HDL-Cholesterol & males $>40 \mathrm{mg} / \mathrm{dl} \mathrm{and} \mathrm{females}>50$ \\
& $\mathrm{mg} / \mathrm{dl}$ \\
Triglycerides & $<150 \mathrm{mg} / \mathrm{dl}$ \\
\hline
\end{tabular}




$$
\begin{aligned}
& \text { FATMass }_{\text {NHANES }}=\text { FATMass }_{\text {Classic }}+0.054 \times \text { LeanSoftTisueMas }_{\text {Classic }} \\
& \text { LeanSoftTisueMasus }_{\text {NHANES }}=\text { TotalMass }- \text { BMC }- \text { FATMass }_{\text {NHANES }}
\end{aligned}
$$

According to Wang et al. (1992), FFM (in kg) was calculated as the difference between body weight $(\mathrm{W}$, in $\mathrm{kg}$ ) and body fat mass measured by the four-component model as the criterion, independent of gender, race, and age.

$$
\begin{aligned}
\mathrm{FFM}=\mathrm{W}-\mathrm{Fat}=\mathrm{W}-(2.749 \mathrm{~V}-0.699 \mathrm{TBW} \\
+1.129 \mathrm{Mo}-2.051 \mathrm{~W})
\end{aligned}
$$

Each DXA scan was performed by a DXA-certified research nurse.

Upon completion of study procedures, participants were given a $\$ 30$ Visa or Walmart gift card in honor of their time and effort, and to help defray and costs (e.g., parking, meals) associated with their participation in the study.

\section{Data Analysis}

An initial descriptive analysis was conducted to review measures of central tendency and assure assumptions regarding adequate variability and normality were met. Because all research questions sought to determine whether relationships existed among various measures of body mass and cardiac risk factors, Pearson Product Moment or Spearman Rho correlation coefficients were determined and used to address all the research questions.

\section{Statistical Assumptions}

There are three statistical assumptions for the Pearson Moment Correlation Coefficient:

1. Independent observations between the participants. The scores for each participant should be independent of all other participants' scores.

2. The $X$ scores should be continuous.

3. Bivariate normality for scores on X and Y. Each participant's scores on Y should be normally distributed in the population $\mathrm{X}$ and $\mathrm{Y}$ should be normally distributed in the population among the $X$ scores.

SAS 9.6 version was used for the data analysis with a-priori $p$-values set at 0.05 . 


\section{Summary}

This chapter described the methods to address the aims of the study and research questions, including the research design, setting and sample, inclusion and exclusion criteria, and the data collection procedures. The analyses used to address the research questions were described along with statistical assumptions. Results of these analyses are presented in Chapter 4. 


\section{CHAPTER 4. RESULTS}

\section{Description of the Sample}

The study sample consisted of 45 participants (see Table 4-1); 29 (64.4\%) were African American and 16 (35.6\%) were White. There were 25 (55.6\%) males and 20 $(44.4 \%)$ females. The age of the sample, at 5 years or longer years posttransplant followup, ranged in age from 37 to 78 with a mean of $56(S D=10.1)$ in Table 4-2. Anthropometric and cardiac-related outcome measures of study participants obtained at the time of transplant and 5 years or more follow-up are presented in Table 4-3 below followed by measures of body composition components at the same measurement points in Table 4-4.

\section{Descriptive Findings}

Tables 4-3 to 4-5 show the descriptive statistics for lab values (creatinine, glucose, $\mathrm{Hgb} \mathrm{A} 1 \mathrm{C}$, triglyceride, HDL, LDL, and coronary risk ratio), elevated blood pressure (systolic and diastolic), BC (VFAT, AFM, GFM, A/G ratio, LM, FM, and \%BF) and anthropometric (BMI, weight, and waist circumference) measurements.

\section{Correlation Findings}

\section{Research Question 1}

Research Question 1 asked, What is the association between anthropometric measures and serum glucose, lipid, and creatinine levels and elevated blood pressure in kidney transplant patients obtained five to eight years posttransplant? Findings that address this question are shown in Table 4-6, which reports the correlational analyses of cardiac-related outcomes and anthropometric measures of BMI, weight, and WC at 5-8 years post-kidney transplant. A significant relationship exists with coronary risk ratio and waist circumference $(r=0.33 ; p=0.05)$ and with diastolic BP and weight $(r=0.28 ; p=$ $0.06)$. Relationships reaching levels of significance $(p \leq 0.20)$ that warrant continued consideration in fully powered studies include weight and $\operatorname{HDL}(r=-0.26 ; p=0.09)$, LDL $(r=-0.22 ; p=0.16)$, and coronary Risk Ratio $(r=-0.28 ; p=0.09)$ as well as waist circumference and diastolic BP $(r=0.30 ; p=0.07)$, and BMI and LDL $(r=-0.21 ; p=$ $0.18)$. These correlations are considered to be weak in strength and positive in direction (Sheskin, 2011). This means that as coronary risk ratio increases so does BMI, weight, and WC. As weight increases so does diastolic BP, HDL, LDL and coronary risk ratio. 
Table 4-1. Demographic Characteristics

\begin{tabular}{ll}
\hline Variables & Total Sample $\boldsymbol{n}(\%)$ \\
\hline Race & \\
White & $16(35.6 \%)$ \\
African-American & $29(64.4 \%)$ \\
Gender & \\
Male & $25(55.6 \%)$ \\
Female & $20(44.4 \%)$ \\
\hline
\end{tabular}

Table 4-2. Age of the Participants

\begin{tabular}{llll}
\hline $\boldsymbol{N}$ & $\boldsymbol{M} \pm \boldsymbol{S D}$ & Min & Max \\
\hline 45 & $56.5 \pm 10.1$ & 37 & 78 \\
\hline
\end{tabular}

Table 4-3. Anthropometric and Cardiac-Related Outcome Measures at 5-8 Years Post-Kidney Transplantation

\begin{tabular}{lccc}
\hline Variables & $\boldsymbol{M} \pm \boldsymbol{S} \boldsymbol{D}$ & Min & Max \\
\hline Anthropometric & & & \\
BMI & $32.14 \pm 9.62$ & 16.21 & 55.95 \\
Weight & $197.89 \pm 48.15$ & 89.80 & 305.80 \\
Waist reading & $41.39 \pm 7.12$ & 23.75 & 54.00 \\
& & & \\
Blood Constituents & & & \\
Creatinine & $1.65 \pm 0.85$ & 0.84 & 5.65 \\
Glucose & $142.41 \pm 91.38$ & 54.00 & 574.00 \\
Hgb AIC & $6.93 \pm 2.22$ & 5.00 & 16.40 \\
Triglyceride & $178.40 \pm 119.38$ & 38.00 & 665.00 \\
HDL & $52.71 \pm 16.85$ & 25.00 & 96.00 \\
LDL & $92.30 \pm 36.57$ & 3.85 & 184.00 \\
Coronary risk ratio & $3.78 \pm 1.31$ & 2.10 & 8.68 \\
Systolic BP & $134.42 \pm 17.85$ & 99.00 & 181.00 \\
Diastolic BP & $78.80 \pm 8.47$ & 59.00 & 98.00 \\
\hline
\end{tabular}

Note . $\mathrm{BMI}=$ body mass index $; \mathrm{Hgb}=$ Hemoglobin $; \mathrm{HDL}=$ high density lipoprotein; $\mathrm{LDL}=$ low density lipoprotein; $\mathrm{BP}=$ blood pressure. 
Table 4-4. Anthropometric, Body Composition, and Cardiac-Related Outcome Measures at Time of Transplant and at 5-8 Years Post-Kidney Transplantation

\begin{tabular}{|c|c|c|c|c|c|c|}
\hline \multirow[b]{2}{*}{ Variables } & \multicolumn{3}{|c|}{ At Time of Transplant } & \multicolumn{3}{|c|}{ 5-8 Years Posttransplant } \\
\hline & $M \pm S D$ & Min & Max & $M \pm S D$ & Min & Max \\
\hline \multicolumn{7}{|l|}{ Anthropometric } \\
\hline BMI & $29.03 \pm 4.76$ & 19.91 & 42.24 & $32.14 \pm 9.61$ & 16.21 & 55.94 \\
\hline Weight & $186.66 \pm 42.10$ & 112.4 & 277.7 & $197.89 \pm 48.1$ & 89.8 & 305.80 \\
\hline \multicolumn{7}{|l|}{ Body Composition } \\
\hline Total Fat & $7334 \pm 2372$ & 2351 & 12099 & $8535 \pm 3377$ & 2002 & 16522 \\
\hline Total Lean & $12238 \pm 2998$ & 6872 & 19647 & $12199 \pm 3413$ & 6353 & 19120 \\
\hline Total PFat & $37.12 \pm 7.76$ & 18.17 & 51.42 & $40.19 \pm 8.10$ & 20.31 & 52.80 \\
\hline Android Fat & $2726 \pm 1101$ & 826.79 & 5215 & $3399 \pm 1676$ & 462.30 & 6823 \\
\hline Android Lean & $4440 \pm 1266$ & 2376 & 8356 & $4301 \pm 1289$ & 2213 & 7348 \\
\hline Android Mass & $7166 \pm 2052$ & 3879 & 11958 & $7700 \pm 2693$ & 3002 & 13130 \\
\hline Android PFat & $37.21 \pm 8.88$ & 17.47 & 54.49 & $42.14 \pm 10.53$ & 15.40 & 56.06 \\
\hline Android/ Gynoid Ratio & $1.01 \pm 0.16$ & 0.71 & 1.38 & $1.08 \pm 0.21$ & 0.6047 & 1.55 \\
\hline Android Percent Fat & $36.73 \pm 9.22$ & 17.47 & 54.49 & $42.14 \pm 10.52$ & 15.39 & 56.06 \\
\hline Gynoid Lean & $7797 \pm 1828$ & 4208 & 11291 & $7898 \pm 2207$ & 4140 & 12035 \\
\hline Gynoid Mass & $12405 \pm 2674$ & 6852 & 16796 & $13034 \pm 3626$ & 6044 & 21895 \\
\hline Gynoid PFat & $36.90 \pm 7.94$ & 18.54 & 53.88 & $38.81 \pm 7.72$ & 22.6 & 52.91 \\
\hline VFat Body Fat & $1428 \pm 608.45$ & 340.28 & 2831 & $1833 \pm 876.70$ & 206.24 & 3411 \\
\hline VFat Body Lean & $2555 \pm 579.06$ & 1400 & 4308 & $2563 \pm 595.90$ & 1260 & 3909 \\
\hline VFat Body Mass & $3983 \pm 998.67$ & 2243 & 5813 & $4396 \pm 1305$ & 1638 & 6690 \\
\hline VFat Body PFat & $34.71 \pm 9.32$ & 13.02 & 51.73 & $39.61 \pm 11.16$ & 10.29 & 53.81 \\
\hline Total Fat Mass & $29590 \pm 9877$ & 10301 & 48639 & $35287 \pm 1587$ & 8121 & 58531 \\
\hline Fat Mass Ratio & $1.03 \pm 0.15$ & 0.70 & 1.40 & $1.09 \pm 0.201$ & 0.73 & 1.534 \\
\hline Total Whole Body Fat & $28508 \pm 9545$ & 7480 & 48639 & $35151 \pm 13362$ & 8121 & 58531 \\
\hline Total Whole Body Lean & $55024 \pm 13063$ & 32189 & 78965 & $54514 \pm 13901$ & 28387 & 81653 \\
\hline Total Whole Body Mass & $83531 \pm 18689$ & 47975 & 120691 & $89665 \pm 23949$ & 40816 & 135562 \\
\hline Total Whole Body PFat & $33.90 \pm 8.12$ & 12.07 & 48.58 & $38.40 \pm 8.41$ & 19.02 & 51.19 \\
\hline
\end{tabular}




\section{Table 4-4. Continued}

Note. $M=$ mean; $\mathrm{S} D=$ standard deviation; $\mathrm{Min}=\operatorname{minimum} ; \mathrm{Max}=\operatorname{maximum} ; \mathrm{BMI}=$ body $\operatorname{mass}$ index $; \mathrm{PFat}=$ percent fat; $\mathrm{VFat}=$ visceral fat. 
Table 4-5. Changes in Anthropometric and Body Composition Measures from Baseline to 5-8 Years Post-Kidney Transplantation

\begin{tabular}{lcll}
\hline Variables & $\boldsymbol{M} \pm \boldsymbol{S D}$ & Min & Max \\
\hline Anthropometric & & & \\
Weight Change & $11.20 \pm 34.33$ & -94.90 & 21.35 \\
BMI Change & $3.05 \pm 7.99$ & -10.08 & \\
DXA Composition & & & 6243 \\
Total Fat & $1201 \pm 2670$ & -7908 & 4081 \\
Total Lean & $-39.26 \pm 1795$ & -4567 & 9669 \\
Total Mass & $1162 \pm 3735$ & -10224 & 15.25 \\
Total PFat & $3.07 \pm 8.13$ & -29.29 & 3516 \\
Android Fat & $672.63 \pm 1317$ & -2743 & 2222 \\
Android Lean & $-138.60 \pm 782.80$ & -2162 & 4794 \\
Android Mass & $534.0 \pm 1705$ & -4886 & 21.73 \\
Android PFat & $4.93 \pm 10.47$ & -31.51 & 0.312 \\
Android Gynoid Ratio & $0.080 \pm 0.15$ & -0.411 & 21.73 \\
Android Percent Fat & $5.411 \pm 9.981$ & -31.51 & 3139 \\
Gynoid Fat & $528.9 \pm 1440$ & -5165 & 2803 \\
Gynoid Lean & $100.20 \pm 1107$ & -2434 & 5412 \\
Gynoid Mass & $629.10 \pm 213$ & -6395 & 13.80 \\
Gynoid PFat & $1.90 \pm 7.19$ & -28.42 & 1537 \\
VFat Body Fat & $405.28 \pm 704.25$ & -1696 & 809.75 \\
VFat Body Lean & $7.86 \pm 323.7$ & -595.42 & 2072 \\
VFat Body Mass & $413.15 \pm 799.02$ & -1914 & 23.10 \\
VFat Body PFat & $4.900 \pm 11.36$ & -34.25 & 22788 \\
Total Fat Mass & $56961 \pm 9325$ & -2537 & 0.267 \\
Fat Mass Ratio & $0.065 \pm 0.116$ & -0.247 & 36866 \\
Total WB Fat Change & $6644 \pm 16424$ & -29458 & 38939 \\
Total WB Lean Change & $-509.79 \pm 19193$ & -40183 & 22.33 \\
Total WB Mass Change & $4.50 \pm 10.31$ & -21.75 & 71662 \\
Total WB PFat Change & $6134 \pm 32149$ & -66194 &
\end{tabular}

Note. $M=$ mean; $\underline{S D}=$ standard deviation; $\mathrm{Min}=$ minimum; $\mathrm{Max}=$ maximum; PFat $=$ percent fat; VFat $=$ visceral fat; $\mathrm{BMI}=$ body mass index; $\mathrm{WB}=$ whole body. 
Table 4-6. Relationship Among Cardiac-Related Outcomes and Anthropometric Measures at 5-8 Years Post-Kidney Transplantation.

\begin{tabular}{llcc}
\hline Variable & $\begin{array}{l}\text { BMI } \\
\boldsymbol{r}(\boldsymbol{p} \text { value })\end{array}$ & $\begin{array}{c}\text { Weight } \\
\boldsymbol{r}(\boldsymbol{p} \text { value }) *\end{array}$ & $\begin{array}{c}\text { Waist Circumference } \\
\boldsymbol{r}(\boldsymbol{p} \text { value }) *\end{array}$ \\
\hline Creatinine & $0.11(0.44)$ & $-0.02(0.88)$ & $-012(0.49)$ \\
Glucose & $0.04(0.98)$ & $0.46(0.80)$ & $0.12(0.47)$ \\
Hgb-AIC & $0.04(0.81)$ & $0.10(0.50)$ & $0.13(0.45)$ \\
Triglyceride & $0.05(0.73)$ & $0.009(1.00)$ & $0.15(0.36)$ \\
HDL & $-0.25(0.10)$ & $-0.26(0.09)$ & $-0.19(0.26)$ \\
LDL & $-0.21(0.18)$ & $-0.22(0.16)$ & $-0.15(0.39)$ \\
Coronary Risk Ratio & $0.27(0.07)$ & $0.28(0.09)$ & $0.33(0.05)$ \\
Systolic BP & $0.05(0.74)$ & $0.15(0.49)$ & $-0.087(0.60)$ \\
Diastolic BP & $0.22(0.15)$ & $0.28(0.06)$ & $0.30(0.07)$ \\
\hline
\end{tabular}

Note. *Pearson Correlation Coefficients: Prob $>[\mathrm{r}]$ under Ho: Rho $=0$ $\mathrm{BMI}=$ body mass index; Hgb = hemoglobin; HDL = high density lipoprotein; LDL = low density lipoprotein; $\mathrm{BP}=$ blood pressure; $r=$ correlation coefficient; $p$ value $\leq 0.05$ and $\leq 0.20$. 


\section{Research Question 2}

Research Question 2 asked, What is the association between body composition measures and serum glucose, lipid, and creatinine levels and elevated blood pressure obtained in kidney transplant recipients 5-8 years posttransplant? Findings that address this question are shown in Tables 4-7 to 4-12, which report the correlational analyses of cardiac-related outcomes and $\mathrm{BC}$ measures by DXA at 5-8 years post-kidney transplant.

The relationship with creatinine and total fat $(r=-0.30 ; p=0.02)$, creatinine and total percent fat $(r=-0.42 ; p=0.004)$, HDL and total lean $(r=-0.40 ; p=0.01)$, and HDL and total mass $(r=-0.16 ; p=0.01)$ are inversely related (Table 4-7). Relationships reaching levels of significance $(p \leq 0.20)$ that warrant continued consideration in fully powered studies include HDL and total fat $(r=-0.30 ; p=0.09)$, LDL and total lean $(r=-$ $21 ; p=0.18)$, coronary risk ratio and total lean $(r=0.27 ; p=0.075)$, coronary risk ratio and total mass $(r=0.26 ; p=0.09)$, systolic and total percent fat $(r=-022 ; p=0.15)$, diastolic and total mass $(r=0.24 ; p=0.12)$, and diastolic and total percent fat $(r=0.25 ; p$ $=0.10)$. These correlations are considered to be weak to moderate in strength. Some are negative, and some are positive in direction (Sheskin, 2011). As total fat and total percentage of fat go up, creatinine goes down and when total lean and total mass go up, HDL goes down. For relationships reaching levels of significance $(p \leq 0.20)$ that warrant continued consideration in fully powered studies, these correlations are also weak in strength. As total fat increases, HDL goes down. As total lean increases, LDL decreases while coronary risk ratio increases. As total mass increases so do coronary risk ratio and diastolic blood pressure. As total percentage of fat increases so does diastolic pressure while systolic blood pressure decreases.

Table 4-8 demonstrates a significant inverse relationship between creatinine and android percentage fat with $(r=-0.45 ; p=0.001)$, HDL and android fat $(r=-0.32 ; p=$ $0.03)$. A significant relationship exists with coronary risk ratio and android lean $(r=0.38$; $p=0.01)$ and coronary risk ratio and android mass $(r=0.35 ; p=0.02)$, and systolic blood pressure and android percent fat $(r=-0.30 ; p=0.05)$. Relationships reaching levels of significance $(p \leq 0.20)$ that warrant continued consideration in fully powered studies include creatinine and android fat $(r=-0.27 ; p=0.07), \mathrm{Hgb} \mathrm{A} 1 \mathrm{C}$ and android fat $(r=$ $0.21 ; p=0.18)$, coronary risk ratio and android fat $(r=0.27 ; p=0.07)$, diastolic and android fat $(r=0.23 ; p=0.13)$, diastolic and android mass $(r=0.22 ; p=0.14)$, and diastolic and android percent fat $(r=0.24 ; p=0.10)$. HDL and android lean $(r=-0.40 ; p$ $=0.006)$, and HDL and android mass $(r=-0.39 ; p=0.008)$ are inversely related. These correlations are considered to be weak in strength. Some are negative, and some are positive in direction (Sheskin, 2011). As android fat goes up, creatinine goes down and when android fat percentage goes up HDL goes down. As coronary risk ratio goes up so do android mass and android lean. For, relationships reaching levels of significance ( $p \leq$ 0.20 ) that warrant continued consideration in fully powered studies, these correlations are also weak in strength. As android fat increases, creatinine goes down; while Hgb A1C goes up. Coronary risk ratio increases as android fat increases. Diastolic blood pressure goes up with android mass and android percent fat. 
Table 4-7. Relationship Among Cardiac-Related Outcomes and Body Composition Measures of Total Fat, Total Lean, Total Mass, and Total Percent Fat at 5-8 Years Post-Kidney Transplantation

\begin{tabular}{lcccc}
\hline Variables & $\begin{array}{c}\text { Total Fat } \\
\boldsymbol{r}(\boldsymbol{p} \text { value })\end{array}$ & $\begin{array}{c}\text { Total Lean- } \\
\boldsymbol{r}(\boldsymbol{p} \text { value }) *\end{array}$ & $\begin{array}{c}\text { Total Mass- } \\
\boldsymbol{r}(\boldsymbol{p} \text { value })\end{array}$ & $\begin{array}{c}\text { Total PFat- } \\
\boldsymbol{r}(\boldsymbol{p} \text { value })^{*}\end{array}$ \\
\hline Creatinine & $-0.30(0.05)$ & $0.06(0.69)$ & $-0.13(0.42)$ & $-0.42(0.004)$ \\
Glucose & $0.13(0.40)$ & $0.08(0.59)$ & $0.12(0.44)$ & $0.13(0.40)$ \\
Hgb AIC & $0.19(0.22)$ & $0.12(0.44)$ & $0.17(0.27)$ & $0.12(0.43)$ \\
Triglyceride & $0.03(0.87)$ & $0.02(0.88)$ & $0.03(0.86)$ & $0.04(0.78)$ \\
HDL & $-0.30(0.09)$ & $-0.40(0.01)$ & $-0.36(0.01)$ & $-0.01(0.93)$ \\
LDL & $-0.08(0.61)$ & $-0.21(0.18)$ & $-0.16(0.31)$ & $0.16(0.32)$ \\
Coronary risk & $0.19(0.22)$ & $0.27(0.08)$ & $0.26(0.09)$ & $0.07(0.67)$ \\
ratio & & & & \\
Systolic BP & $-0.13(0.40)$ & $0.08(0.59)$ & $-0.02(0.87)$ & $-0.22(0.15)$ \\
Diastolic BP & $0.27(0.78)$ & $0.167(0.27)$ & $0.24(0.12)$ & $0.25(0.10)$ \\
\hline
\end{tabular}

Note. *Pearson Correlation Coefficients: Prob $>[\mathrm{r}]$ under Ho: Rho $=0$; $\mathrm{Hgb}=$ hemoglobin; $\mathrm{HDL}=$ high density lipoprotein; $\mathrm{LDL}=$ low density lipoprotein; $\mathrm{BP}=$ blood pressure; PFat $=$ percent fat; $r=$ correlation coefficient; $p$ value $\leq 0.05$ and $\leq 0.20$.

Table 4-8. Relationship Among Cardiac-Related Outcomes and Body Composition Measures of Android Fat, Android Lean, Android Mass, and Android Percent Fat at 5-8 Years Post-Kidney Transplantation

\begin{tabular}{lcccc}
\hline Variables & $\begin{array}{c}\text { Android } \\
\text { Fat } \\
\boldsymbol{r}(\boldsymbol{p} \text { value }) *\end{array}$ & $\begin{array}{c}\text { Android } \\
\text { Lean } \\
\boldsymbol{r}(\boldsymbol{p} \text { value }) *\end{array}$ & $\begin{array}{c}\text { Android } \\
\text { Mass } \\
\boldsymbol{r}(\boldsymbol{p} \text { value }) *\end{array}$ & $\begin{array}{c}\text { Android } \\
\text { PFat } \\
\boldsymbol{r}(\boldsymbol{p} \text { value }) *\end{array}$ \\
\hline Creatinine & $-0.27(0.07)$ & $0.12(0.43)$ & $-0.11(0.48)$ & $-0.45(0.001)$ \\
Glucose & $0.16(0.31)$ & $0.10(0.64)$ & $0.15(0.34)$ & $0.15(0.33)$ \\
Hgb A1C & $0.21(0.18)$ & $0.10(0.51)$ & $0.18(0.25)$ & $0.16(0.30)$ \\
Triglyceride & $0.11(048)$ & $0.15(0.32)$ & $0.14(0.36)$ & $0.09(0.55)$ \\
HDL & $-0.32(0.03)$ & $-0.40(0.006)$ & $-0.39(0.008)$ & $-0.14(0.37)$ \\
LDL & $-0.14(0.37)$ & $-0.18(0.25)$ & $-0.17(0.27)$ & $0.09(0.60)$ \\
Coronary risk ratio & $0.27(0.07)$ & $0.38(0.01)$ & $0.35(0.02)$ & $0.15(0.29)$ \\
Systolic BP & $-0.16(0.28)$ & $0.14(0.34)$ & $-0.03(0.87)$ & $-0.30(0.05)$ \\
Diastolic BP & $0.23(0.13)$ & $0.16(0.29)$ & $0.22(0.14)$ & $0.24(0.10)$ \\
\hline
\end{tabular}

Note. *Pearson Correlation Coefficients: Prob > [r] under Ho: Rho $=0 ; \mathrm{Hgb}=$ hemoglobin; HDL = high density lipoprotein; $\mathrm{LDL}=$ low density lipoprotein; $\mathrm{BP}=$ blood pressure; PFat $=$ percent fat; $r=$ correlation coefficient; $p$ value $\leq 0.05$ and $\leq 0.20$. 
Table 4-9. Relationship Among Android Gynoid Ratio and Cardiac-Related Outcomes at 5-8 Years Post-Kidney Ttransplantation

\begin{tabular}{lc}
\hline Variables & $\begin{array}{c}\text { Android Gynoid Ratio } \\
\boldsymbol{r}(\boldsymbol{p} \text { value }) * *\end{array}$ \\
\hline Creatinine & $-0.28(0.06)$ \\
Glucose & $0.13(0.39)$ \\
Hgb A1C & $0.14(0.38)$ \\
Triglyceride & $0.09(0.56)$ \\
HDL & $-0.27(0.07)$ \\
LDL & $-0.06(0.71)$ \\
Coronary risk ratio & $0.21(0.71)$ \\
Systolic BP & $-0.29(0.05)$ \\
Diastolic BP & $0.12(0.5)$ \\
\hline
\end{tabular}

Note. **Pearson Correlation Coefficients: Prob $>[\mathrm{r}]$ under Ho: Rho $=0$; Hgb A1C $=$ hemoglobin A1C; HDL = high density lipoprotein; LDL = low density lipoprotein; $\mathrm{BP}=$ blood pressure; $r=$ correlation coefficient; $p$ value $\leq 0.05$ and $\leq 0.20$.

Table 4-10. Relationship Among Cardiac-Related Outcome and Body Composition Measures of Gynoid Fat, Gynoid Lean, Gynoid Mass, and Gynoid Percent Fat at 5-8 Years Post-Kidney Transplantation

\begin{tabular}{lcccc}
\hline Variables & $\begin{array}{c}\text { Gynoid Fat } \\
\boldsymbol{r}(\boldsymbol{p} \text { value })\end{array}$ & $\begin{array}{c}\text { Gynoid Lean } \\
\boldsymbol{r}(\boldsymbol{p} \text { value }) *\end{array}$ & $\begin{array}{c}\text { Gynoid Mass } \\
\boldsymbol{r}(\boldsymbol{p} \text { value)*}\end{array}$ & $\begin{array}{c}\text { Gynoid PFat } \\
\boldsymbol{p} \text { value })^{*}\end{array}$ \\
\hline Creatinine & $-0.28(0.06)$ & $0.02(0.88)$ & $-0.13(0.39)$ & $-0.34(0.02)$ \\
Glucose & $0.09(0.55)$ & $0.07(0.65)$ & $0.09(0.56)$ & $0.09(0.54)$ \\
Hgb A1C & $0.15(0.32)$ & $0.13(0.41)$ & $0.16(0.31)$ & $0.08(0.62)$ \\
Triglyceride & $-0.05(0.74)$ & $-0.06(0.72)$ & $-0.06(0.69)$ & $-0.02(0.92)$ \\
HDL & $-0.18(0.24)$ & $-0.38(0.10)$ & $-0.32(0.03)$ & $0.09(0.54)$ \\
LDL & $-0.02(0.91)$ & $-0.22(0.16)$ & $-0.14(0.36)$ & $0.20(0.20)$ \\
Coronary & $0.10(0.52)$ & $0.20(0.20)$ & $0.17(0.26)$ & $-0.003(0.98)$ \\
risk ratio & & & & \\
Systolic BP & $-0.09(0.58)$ & $0.04(0.77)$ & $-0.02(0.91)$ & $-0.12(0.42)$ \\
Diastolic BP & $0.27(0.07)$ & $0.16(0.28)$ & $0.24(0.11)$ & $0.23(0.14)$ \\
\hline
\end{tabular}

Note. *Pearson Correlation Coefficients: Prob $>[\mathrm{r}]$ under Ho: $\mathrm{Rho}=0 ; \mathrm{Hgb}=$ hemoglobin; $\mathrm{HDL}=$ high density lipoprotein; $\mathrm{LDL}=$ low density lipoprotein; $\mathrm{PFat}=$ percent fat; $\mathrm{BP}=$ blood pressure; $r=$ correlation coefficient; $p$ value $\leq 0.05$ and $\leq 0.20$. 
Table 4-11. Relationship Among Cardiac-Related Outcome and Body

Composition Measures of VFat, VBody Lean, VBody Mass, and VPercent Fat at 5-8 Years Post-Kidney Transplantation

\begin{tabular}{lcccc}
\hline Variables & $\begin{array}{c}\text { VFat } \\
\boldsymbol{r}(\boldsymbol{p} \text { value }) *\end{array}$ & $\begin{array}{c}\text { VFat Body } \\
\text { Lean } \\
\boldsymbol{r}(\boldsymbol{p} \text { value }) *\end{array}$ & $\begin{array}{c}\text { VFat Body } \\
\text { Mass } \\
\boldsymbol{r}(\boldsymbol{p} \text { value }) *\end{array}$ & $\begin{array}{c}\text { VFat Body } \\
\text { PFat }\end{array}$ \\
\hline Creatinine- & $\boldsymbol{p}$ value $)$ \\
Glucose & $-0.30(0.05)$ & $0.20(0.20)$ & $-0.17(0.49)$ & $-0.47(0.001)$ \\
Hgb A1C & $0.16(0.30)$ & $0.15(0.33)$ & $0.18(0.25)$ & $0.15(0.34)$ \\
Triglyceride & $0.18(0.23)$ & $0.10(0.50)$ & $0.17(0.27)$ & $0.15(0.33)$ \\
HDL & $0.07(0.64)$ & $0.15(0.33)$ & $0.12(0.44)$ & $0.06(0.72)$ \\
LDL & $-0.28(0.07)$ & $-0.37(0.02)$ & $-0.34(0.02)$ & $-0.15(0.33)$ \\
Coronary risk ratio & $-0.09(0.58)$ & $-0.19(0.38)$ & $-0.12(0.45)$ & $0.09(0.55)$ \\
Systolic BP & $0.23(0.13)$ & $0.37(0.01)$ & $0.32(0.03)$ & $0.15(0.34)$ \\
Diastolic BP & $-0.19(0.21)$ & $0.17(0.26)$ & $-0.05(0.74)$ & $-0.29(0.05)$ \\
\hline
\end{tabular}

Note. *Pearson Correlation Coefficients: Prob $>[\mathrm{r}]$ under Ho: Rho=0;Hgb $=$ hemoglobin; $\mathrm{HDL}=$ high density lipoprotein; $\mathrm{LDL}=$ low density lipoprotein; VFat $=$ visceral fat; $\mathrm{BP}$ $=$ blood pressure; PFat $=$ percent fat; $r=$ correlation coefficient; $p$ value $\leq 0.05$ and $\leq$ 0.20 . 
Table 4-12. Relationship Among Cardiac-Related Outcomes and Body Composition Measures of Whole Body Total Fat, Whole Body Total Lean, Whole Body Total Mass, and Whole Body Total Percent Fat at 5-8 Years Post-Kidney Transplantation

\begin{tabular}{lllll}
\hline Variables & $\begin{array}{c}\text { WBTotal Fat } \\
\boldsymbol{r}(\boldsymbol{p} \text { value }) *\end{array}$ & $\begin{array}{c}\text { WBTotal } \\
\text { Lean- } \\
\boldsymbol{r}(\boldsymbol{p} \text { value }) *\end{array}$ & $\begin{array}{c}\text { WBTotal } \\
\text { Mass- } \\
\boldsymbol{r}(\boldsymbol{p} \text { value }) *\end{array}$ & $\begin{array}{c}\text { WBTotal } \\
\text { PFat- } \\
\boldsymbol{r}(\boldsymbol{p} \text { value })\end{array}$ \\
\hline Creatinine & $-0.24(0.11)$ & $-0.05(0.76)$ & $-0.16(0.28)$ & $-0.26(0.08)$ \\
Glucose & $-0.28(0.07)$ & $-0.11(0.49)$ & $-0.22(0.15)$ & $-0.26(0.09)$ \\
Hgb AIC & $-0.27(0.08)$ & $-0.11(0.46)$ & $-0.22(0.16)$ & $-0.23(0.14)$ \\
Triglyceride & $0.11(0.48)$ & $0.15(0.33)$ & $0.15(0.33)$ & $0.01(0.92)$ \\
HDL & $0.16(0.31)$ & $0.12(0.44)$ & $0.15(0.44)$ & $0.04(0.80)$ \\
LDL & $-0.13(0.44)$ & $-0.06(0.69)$ & $-0.11(0.49)$ & $-0.14(0.39)$ \\
Coronary risk ratio & $-0.04(0.81)$ & $0.02(0.88)$ & $-0.01(0.97)$ & $-0.06(0.68)$ \\
Systolic BP & $-0.27(0.07)$ & $-0.43(<0.001)$ & $-0.40(0.01)$ & $-0.08(0.61)$ \\
Diastolic BP & $-0.23(0.16)$ & $-0.20(0.20)$ & $-0.24(0.11)$ & $-0.15(0.32)$ \\
\hline
\end{tabular}

Note. $\mathrm{WB}=\mathrm{Whole}-$ body; $\mathrm{Hgb}=$ hemoglobin; $\mathrm{HDL}=$ high density lipoprotein LDL $=1$ density lipoprotein; PFat = percent fat; $\mathrm{BP}=$ blood pressure; $r=$ correlation coefficient; $p$ value $\leq 0.05$ and $\leq 0.20$. 
An inverse relationship with significance exists with systolic BP and android gynoid ratio $(r=-0.29 ; p=0.05)$. The relationships between glucose and $\mathrm{Hgb} \mathrm{A} 1 \mathrm{C}(r=$ $0.91 ; p=0.001)$ and triglyceride and $\operatorname{Hgb~A1C~}(r=0.225 ; p=0.137)$ are both significant (Table 4-9). Relationships reaching levels of significance $(p \leq 0.20)$ that warrant continued consideration in fully powered studies include creatinine and android gynoid ratio $(r=-0.28 ; p=0.06)$ and HDL and android gynoid ratio $(r=-0.27 ; p=0.07)$. These correlations are considered weak to strong in strength and some are negative, and some are positive in direction (Sheskin, 2011). As the android gynoid ratio increases, systolic blood pressure decreases. For relationships reaching levels of significance $(p \leq 0.20)$, as android gynoid ratio increases, creatinine and HDL decrease.

Table 4-10 demonstrates a significant relationship with an inverse relationship existing with creatinine and gynoid percent fat $(r=-0.34 ; p=0.02)$ and HDL and gynoid mass $(r=-0.32 ; p=0.03)$, which are presented in Table 4-10. Relationships reaching levels of significance $(p \leq 0.09)$ that warrant continued consideration in fully powered studies include creatinine and gynoid fat $(r=-0.28 ; p=0.06)$, and diastolic and gynoid fat $(r=0.27 ; p=0.07)$.

Table 4-11 demonstrates a significant relationship with coronary risk ratio and visceral fat body lean $(r=0.37 ; p=0.01)$, and coronary risk ratio and visceral fat body mass $(r=0.32 ; p=0.03)$. An inverse relationship exists with creatinine and visceral fat $(r$ $=-0.30 ; p=0.05)$, HDL and visceral fat body lean $(r=-0.34 ; p=0.02)$, HDL and visceral fat body mass $(r=-0.34 ; p=0.02)$, and systolic pressure and visceral fat body percent fat $(r=-0.29 ; p=0.05)$. Relationships reaching levels of significance $(p \leq 0.20)$ that warrant continued consideration in fully powered studies include creatinine and visceral fat body lean $(r=0.20 ; p=0.20)$, HDL and visceral fat $(r=-0.28 ; p=0.07)$, coronary risk ratio $(r=0.23 ; p=0.13)$, diastolic and visceral fat $(r=0.26 ; p=0.09)$, diastolic BP and visceral fat body mass $(r=0.26 ; p=0.09)$, and diastolic BP and visceral fat body percent fat $(r=0.26 ; p=0.09)$. These correlations were weak in strength. For relationships reaching levels of significance $(p \leq 0.20)$, as visceral fat body lean increases, so does creatinine. As visceral fat increases HDL decreases and coronary risk ratio and diastolic BP increase. Diastolic pressure also increases as visceral fat body mass and visceral fat body percent increase. These correlations are considered to be weak in strength and are positive (Sheskin, 2011).

The relationships with WB total lean and systolic BP $(r=-0.43 ; p=<0.001)$ and WB total mass and systolic BP $(r=-0.40 ; p=0.01)$ are inversely related (Table 4-12). In addition, an inverse relationship reaching significance of $(\mathrm{p} \leq 0.20)$ was found with systolic and WB total fat $(r=-0.27 ; p=(0.07)$. An inverse relationship with creatinine and WB total fat $(r=-0.24 ; \mathrm{p}=0.11)$, creatinine and WB total PFat $(r=-0.26 ; p=0.08)$; and glucose and WB total fat $(r=-0.28 ; p=0.07)$, glucose and WB total mass $(\mathrm{r}=-.022$; $p=0.15)$, and glucose and WB total PFat $(r=-0.26 ; p=0.09$. These correlations are considered to be weak in strength and negative in direction. As systolic BP increases, WB total lean, WB total mass, and WB total fat decrease. For relationships reaching levels of significance $(p \leq 0.20)$ that warrant continued consideration in fully powered studies, these correlations are considered weak in strength and negative in direction. An inverse 
relationship with $\mathrm{HgbA1C}$ and WB total fat $(r=-0.27 ; p=0.08)$; HgbA1C and WB total mass $(r=-0.22 ; p=0.16)$; and HgbA1c and WB total PFat $(r=-0.23 ; p=0.14)$; diastolic $\mathrm{BP}$ and WB total fat $(r=-0.23 ; p=0.16)$, diastolic BP and WB total lean $(\mathrm{r}=-0.20 ; p=$ $0.20)$; diastolic $\mathrm{BP}$ and WB total mass $(\mathrm{r}=-0.24 ; p=0.11)$. As WB total fat and WB total PFat go up, the creatinine and glucose levels go down. The glucose level also goes down when WB total mass goes up. As diastolic BP and HgbA1C go up, WB total fat and WB total pfat go down. Diastolic BP also goes down when WB total lean values increase. As HgbA1c decreases, so does WB total mass.

\section{Research Question 3}

Research Question 3 asked, To what degree are body composition measures obtained at the time of transplant associated with serum levels of glucose, lipids, and creatinine and elevated blood pressure in kidney transplant recipients at 5 to 8 years posttransplant? Tables 4-13 to 4-18 report the correlational analyses of cardiac-related outcomes and $\mathrm{BC}$ measures at time of transplant is associated with recipients at 5-8 years post-kidney transplant.

An inverse relationship with significance was found with HDL and total fat $(\mathrm{r}=$ 0.34; $p=0.02)$, HDL and total lean $(r=-0.50 ; p=<0.001)$, and HDL and total mass $(r=$ $-0.51 ; p=0.0004)$. A significant relationship exists with coronary risk ratio and total lean $(r=0.42 ; p=0.004)$ and coronary risk ratio and total percent fat $(r=0.38 ; p=0.01)$

(Table 4-13). Relationships reaching levels of significance $(p \leq 0.20)$ that warrant continued consideration in fully powered studies include glucose and total fat $(r=0.20 ; p$ $=0.18), \mathrm{LDL}$ and total fat $(r=-0.20 ; p=0.20)$, and coronary risk ratio $(r=0.25 ; p=$ $0.24)$. Relationships reaching levels of significance $(p \leq 0.20)$ that warrant continued consideration in fully powered studies include as glucose levels increase so does total fat, and as LDL increases, so does total fat.

A significant relationship was found with triglyceride and android lean $(r=0.30$; $p=0.050)$, coronary risk ratio and android fat $(r=0.32 ; p=0.03)$, and coronary risk ratio and android mass $(r=0.47 ; p=0.001)$. An inverse relationship exists with HDL and android fat $(r=-0.46 ; p=0.001)$, HDL and android lean $(r=-0.45 ; p=0.002)$ and HDL and android mass $(r=-0.53 ; p=<0.001)$; Table 4-14). Relationships reaching levels of significance $(p \leq 0.20)$ that warrant continued consideration in fully powered studies include glucose and android fat $(r=0.24 ; p=0.12)$, Hgb A1C and android fat $(r=0.21 ; p$ $=0.16)$, and triglyceride and android mass $(r=0.28 ; p=0.07)$. The correlations range from weak to moderate in strength. As android lean increases so do triglyceride levels; coronary risk ratio increases with android fat, android, and android mass increases. HDL decreases as android lean, fat, and mass increase. For relationships reaching levels of significance $(p \leq 0.20)$, glucose and $\mathrm{HgbA} 1 \mathrm{C}$ go up with android fat, and triglycerides go up with android mass. 
Table 4-13. Relationship Among Cardiac-Related Outcomes at 5-8 Years PostKidney Transplantation and Body Composition Measures of Total Fat, Total Lean, Total Mass, and Total Percent Fat Obtained at Time of Transplant

\begin{tabular}{lcccc}
\hline Variables & $\begin{array}{c}\text { Total Fat- } \\
\boldsymbol{r}(\boldsymbol{p} \text { value })\end{array}$ & $\begin{array}{c}\text { Total Lean } \\
\boldsymbol{r}(\boldsymbol{p} \text { value })\end{array}$ & $\begin{array}{c}\text { Total Mass } \\
\boldsymbol{r}(\boldsymbol{p} \text { value })\end{array}$ & $\begin{array}{c}\text { Total PFat } \\
\boldsymbol{r}(\boldsymbol{p} \text { value })\end{array}$ \\
\hline Creatinine & $-0.03(0.86)$ & $0.11(0.46)$ & $0.061(0.69)$ & $-0.13(0.39)$ \\
Glucose & $0.20(0.18)$ & $0.11(0.48)$ & $0.18(0.25)$ & $0.12(0.44)$ \\
Hgb A1C & $0.19(0.21)$ & $0.07(0.63)$ & $0.01(0.33)$ & $0.11(0.46)$ \\
Triglyceride & $0.08(0.61)$ & $0.18(0.24)$ & $0.16(0.29)$ & $-0.03(0.86)$ \\
HDL & $-0.34(0.02)$ & $-0.50(<0.001)$ & $-0.51(0.0004)$ & $-0.01(0.96)$ \\
LDL & $-0.20(0.20)$ & $0.01(0.95)$ & $-0.12(0.46)$ & $-0.16(0.30)$ \\
Coronary risk ratio & $0.25(0.24)$ & $0.42(0.004)$ & $0.38(0.01)$ & $-0.09(0.54)$ \\
Systolic BP & $0.05(0.76)$ & $0.08(0.61)$ & $0.075(0.625)$ & $-0.04(0.79)$ \\
Diastolic BP & $0.07(0.66)$ & $0.09(0.54)$ & $0.096(0.528)$ & $-0.03(0.86)$ \\
\hline
\end{tabular}

Note. *Pearson Correlation Coefficients: Prob $>[\mathrm{r}]$ under Ho: Rho=0; Hgb $=$ hemoglobin; HDL = high density lipoprotein; $\mathrm{LDL}=1$ density lipoprotein; $\mathrm{PFat}=$ percent fat; $\mathrm{BP}=$ blood pressure; $r=$ correlation coefficient; $p$ value $\leq 0.05$ and $\leq 0.20$.

Table 4-14. Relationships Among Cardiac-Related Outcomes at 5-8 Years PostKidney Transplantation and Body Composition Measures of Android Fat, Android Lean, Android Mass, and Android Percent Fat Obtained at Time of Transplant

\begin{tabular}{lcccc}
\hline Variables & $\begin{array}{c}\text { Android Fat } \\
\boldsymbol{r}(\boldsymbol{p} \text { value })\end{array}$ & $\begin{array}{c}\text { Android Lean } \\
\boldsymbol{r}(\boldsymbol{p} \text { value })^{*}\end{array}$ & $\begin{array}{c}\text { Android Mass } \\
\boldsymbol{r}(\boldsymbol{p} \text { value })^{*}\end{array}$ & $\begin{array}{c}\text { Android PFat } \\
\boldsymbol{r}(\boldsymbol{p} \text { value })^{*}\end{array}$ \\
\hline Creatinine & $-0.01(0.97)$ & $0.07(0.63)$ & $0.04(0.78)$ & $-0.09(0.58)$ \\
Glucose & $0.24(0.12)$ & $0.08(0.60)$ & $0.18(0.25)$ & $0.17(0.28)$ \\
Hgb A1C & $0.21(0.16)$ & $0.03(0.81)$ & $0.14(0.38)$ & $0.16(0.30)$ \\
Triglyceride & $0.17(0.26)$ & $0.30(0.05)$ & $0.30(0.07)$ & $-0.10(0.51)$ \\
HDL & $-0.46(0.001)$ & $-0.45(0.002)$ & $-0.53(<0.001)$ & $-0.19(0.22)$ \\
LDL & $-0.19(0.23)$ & $-0.023(0.88)$ & $-0.09(0.56)$ & $-0.16(0.31)$ \\
Coronary risk ratio & $0.32(0.03)$ & $0.32(0.008)$ & $0.47(0.001)$ & $-0.03(0.83)$ \\
Systolic BP & $0.03(0.82)$ & $0.07(0.64)$ & $0.06(0.68)$ & $-0.04(0.78)$ \\
Diastolic BP & $0.01(0.97)$ & $0.023(0.88)$ & $0.02(0.91)$ & $-0.01(0.90)$ \\
\hline
\end{tabular}

Note. ${ }^{*}$ Pearson Correlation Coefficients: Prob $>[\mathrm{r}]$ under Ho: Rho $=0 ; \mathrm{Hgb}=$ hemoglobin; HDL = high density lipoprotein; LDL = low density lipoprotein; PFat = percent fat; $\mathrm{BP}=$ blood pressure; $r=$ correlation coefficient; $p$ value $\leq 0.05$ and $\leq 0.20$. 
Table 4-15. Relationship Among Cardiac-Related Outcomes at 5-8 Years PostKidney Transplantation and Body Composition Measures of Gynoid Fat, Gynoid Lean, Gynoid Mass, and Gynoid Percent Fat Obtained at Time of Transplant

\begin{tabular}{lcccc}
\hline Variables & $\begin{array}{c}\text { Gynoid Fat } \\
\boldsymbol{r}(\boldsymbol{p} \text { value })\end{array}$ & $\begin{array}{c}\text { Gynoid Lean } \\
\boldsymbol{r}(\boldsymbol{p} \text { value })^{*}\end{array}$ & $\begin{array}{c}\text { Gynoid Mass } \\
\boldsymbol{r}(\boldsymbol{p} \text { value })^{*}\end{array}$ & $\begin{array}{c}\text { Gynoid PFat } \\
\boldsymbol{r}(\boldsymbol{p} \text { value })^{*}\end{array}$ \\
\hline Creatinine & $-0.04(0.79)$ & $0.14(0.38)$ & $0.07(0.64)$ & $-0.15(0.32)$ \\
Glucose & $0.15(0.32)$ & $0.12(0.44)$ & $0.17(0.28)$ & $0.08(0.61)$ \\
Hgb A1C & $0.16(0.31)$ & $0.094(0.54)$ & $0.15(0.33)$ & $0.08(0.61)$ \\
Triglyceride & $-0.002(1.0)$ & $0.093(0.55)$ & $0.06(0.68)$ & $-0.04(0.81)$ \\
HDL & $-0.21(0.18)$ & $-0.51<0.001)$ & $-0.46(0.001)$ & $0.14(0.36)$ \\
LDL & $-0.19(0.23)$ & $-0.03(0.83)$ & $-0.13(0.41)$ & $-0.14(0.37)$ \\
Coronary risk ratio & $0.06(0.72)$ & $0.36(0.02)$ & $0.28(0.07)$ & $-0.16(0.29)$ \\
Systolic BP & $0.05(0.74)$ & $0.08(0.62)$ & $0.08(0.60)$ & $-0.03(0.86)$ \\
Diastolic BP & $0.10(0.49)$ & $0.14(0.36)$ & $0.15(0.32)$ & $-0.02(0.90)$ \\
\hline
\end{tabular}

Note. *Pearson Correlation Coefficients: Prob $>[\mathrm{r}]$ under Ho: Rho $=0 ; \mathrm{Hgb}=$ hemoglobin; HDL = high density lipoprotein; LDL = low density; $\mathrm{BP}=$ blood pressure; PFat $=$ percent fat; $r=$ correlation coefficient; $p$ value $\leq 0.05$ and $\leq 0.20$.

Table 4-16. Relationship Among Cardiac-Related Outcomes at 5-8 Years PostKidney Transplantation and Body Composition Measures of Visceral Fat Body Fat, Visceral Fat Body Lean, Visceral Fat Body Mass, and Visceral Fat Body Percent Fat Obtained at Time of Transplant

\begin{tabular}{lcccc}
\hline Variables & $\begin{array}{c}\text { VFat Body } \\
\text { Fat } \\
\boldsymbol{r}(\boldsymbol{p} \text { value })\end{array}$ & $\begin{array}{c}\text { VFat Body } \\
\text { Lean } \\
\boldsymbol{r}(\boldsymbol{p} \text { value })\end{array}$ & $\begin{array}{c}\text { VFat Body } \\
\text { Mass } \\
\boldsymbol{r}(\boldsymbol{p} \text { value })\end{array}$ & $\begin{array}{c}\text { VFat Body } \\
\text { PFat } \\
\boldsymbol{r}(\boldsymbol{p} \text { value })\end{array}$ \\
\hline Creatinine & $-0.003(0.98)$ & $0.13(0.40)$ & $0.07(0.64)$ & $-0.09(0.54)$ \\
Glucose & $0.19(0.22)$ & $0.08(0.61)$ & $0.16(0.30)$ & $0.16(0.31)$ \\
Hgb A1C- & $0.16(0.31)$ & $-0.01(1.00)$ & $0.09(0.56)$ & $0.15(0.32)$ \\
Triglyceride & $0.11(0.46)$ & $0.32(0.04)$ & $0.25(0.10)$ & $-0.01(0.96)$ \\
HDL & $-0.37(0.01)$ & $-0.34(0.02)$ & $-0.43(0.003)$ & $-0.21(0.17)$ \\
LDL & $-0.18(0.26)$ & $0.055(0.73)$ & $-0.08(0.61)$ & $-0.16(0.32)$ \\
Coronary risk ratio & $0.26(0.09)$ & $0.52(0.003)$ & $0.45(0.002)$ & $-0.04(0.80)$ \\
Systolic BP & $0.04(0.79)$ & $0.13(0.38)$ & $0.10(0.50)$ & $-0.04(0.80)$ \\
Diastolic BP & $0.05(0.74)$ & $0.13(0.40)$ & $0.10(0.50)$ & $-0.01(0.90)$ \\
\hline
\end{tabular}

Note. *Pearson Correlation Coefficients: Prob $>[\mathrm{r}]$ under Ho: Rho $=0 ; \mathrm{Hgb}=$ hemoglobin; $\mathrm{HDL}=$ high density lipoprotein; $\mathrm{LDL}=$ low density lipoprotein; $\mathrm{BP}=$ blood pressure; VFat $=$ visceral fat; PFat $=$ percent fat; $r=$ correlation coefficient; $p$ value $\leq$ 0.05 and $\leq 0.20$. 
Table 4-17. Relationship Among Cardiac-Related Outcomes at 5-8 Years PostKidney Transplantation and Body Composition Measures of Android Gynoid Ratio, Android Percent Fat, Total Fat Mass, and Fat Mass Ratio Obtained at Time of Transplant

\begin{tabular}{lcccc}
\hline Variables & $\begin{array}{c}\text { Android/ } \\
\text { Gynoid Ratio } \\
\boldsymbol{r}(\boldsymbol{p} \text { value })^{*}\end{array}$ & $\begin{array}{c}\text { Android } \\
\text { Percent Fat } \\
\boldsymbol{r}(\boldsymbol{p} \text { value })^{*}\end{array}$ & $\begin{array}{c}\text { Total Fat } \\
\text { Mass } \\
\boldsymbol{r}(\boldsymbol{p} \text { value })^{*}\end{array}$ & $\begin{array}{c}\text { Fat Mass } \\
\text { Ratio } \\
\boldsymbol{r}(\boldsymbol{p} \text { value }) *\end{array}$ \\
\hline Creatinine & $0.01(0.97)$ & $-0.14(0.35)$ & $-0.05(0.74)$ & $-0.06(0.68)$ \\
Glucose & $0.15(0.34)$ & $0.18(0.23)$ & $0.28(0.061)$ & $0.20(0.20)$ \\
Hgb A1C & $0.16(0.30)$ & $0.20(0.19)$ & $0.26(0.09)$ & $0.21(0.17)$ \\
Triglyceride & $0.02(0.89)$ & $-0.003(0.98)$ & $0.13(0.41)$ & $0.08(0.60)$ \\
HDL & $-0.46(0.001)$ & $-0.15(0.33)$ & $-0.28(0.06)$ & $-0.39(0.007)$ \\
LDL & $-0.09(0.57)$ & $-0.17(0.27)$ & $-0.21(0.17)$ & $-0.10(0.54)$ \\
Coronary risk ratio & $0.23(0.13)$ & $0.01(0.94)$ & $0.21(0.16)$ & $0.20(0.19)$ \\
Systolic BP & $-0.04(0.81)$ & $-0.04(0.79)$ & $0.05(0.75)$ & $-0.03(0.85)$ \\
Diastolic BP & $0.09(0.56)$ & $0.09(0.54)$ & $0.15(0.33)$ & $0.09(0.57)$ \\
\hline
\end{tabular}

Note. *Pearson Correlation Coefficients: Prob $>[\mathrm{r}]$ under Ho: Rho $=0$

$\mathrm{Hgb}=$ hemoglobin; HDL = high density lipoprotein; LDL = low density lipoprotein; BP $=$ blood pressure; $r=$ correlation coefficient; $p$ value $\leq 0.05$ and $\leq 0.20$. 
Table 4-18. Relationship Among Cardiac-Related Outcomes at 5-8 Years PostKidney Transplantation and Body Composition Measures of Whole Body Total Fat, Whole Body Total Lean, Whole Body Total Mass, and Whole Body Total Percent Fat Obtained at Time of Transplant

\begin{tabular}{lcccc}
\hline Variable & $\begin{array}{c}\text { WBTotal Fat } \\
\boldsymbol{r}(\boldsymbol{p} \text { value })\end{array}$ & $\begin{array}{c}\text { WBTotal } \\
\text { Lean- } \\
\boldsymbol{r}(\boldsymbol{p} \text { value }) *\end{array}$ & $\begin{array}{c}\text { WBTotal } \\
\text { Mass- } \\
\boldsymbol{r}(\boldsymbol{p} \text { value }) *\end{array}$ & $\begin{array}{c}\text { WBTotal } \\
\text { PFat- } \\
\boldsymbol{r}(\boldsymbol{p} \text { value }) *\end{array}$ \\
\hline Creatinine & $-0.09(0.53)$ & $0.13(0.39)$ & $0.04(0.77)$ & $-0.20(0.20)$ \\
Glucose & $0.31(0.04)$ & $0.12(0.44)$ & $0.24(0.11)$ & $0.20(0.20)$ \\
HgbA1C & $0.30(0.05)$ & $0.09(.56)$ & $0.21(0.16)$ & $0.20(0.20)$ \\
Triglyceride & $0.12(0.44)$ & $0.13(0.39)$ & $0.15(0.31)$ & $0.03(0.83)$ \\
HDL & $-0.27(0.07)$ & $-0.45(0.002)$ & $-0.45(0.002)$ & $0.04(0.79)$ \\
LDL & $-0.22(0.15)$ & $-0.06(0.72)$ & $-0.16(0.32)$ & $-0.15(0.33)$ \\
Coronary risk & $0.20(0.20)$ & $0.41(0.001)$ & $0.39(0.001)$ & $-0.07(0.66)$ \\
ratio & & & & \\
Systolic BP & $0.05(0.73)$ & $0.12(0.41)$ & $0.11(0.45)$ & $-0.03(0.85)$ \\
Diastolic BP & $0.15(0.34)$ & $0.19(0.22)$ & $0.20(0.18)$ & $0.02(0.91)$ \\
\hline
\end{tabular}

Note. *Pearson Correlation Coefficients: Prob $>[\mathrm{r}]$ under Ho: Rho=0; WB $=$ Whole-body; $\mathrm{Hgb}=$ hemoglobin; HDL = high density lipoprotein; LDL = low density lipoprotein; BP $=$ blood pressure; PFat $=$ percent fat; $r=$ correlation coefficient; $p$ value $\leq 0.05$ and $\leq 0.20$ 
Table 4-15 shows an inverse relationship exists with HDL and gynoid lean $(r=$ $0.51 ; p=<0.001)$ and HDL and gynoid mass $(r=-0.46 ; p=0.001)$. The relationship with coronary risk ratio and gynoid lean $(r=0.36 ; p=0.02)$ was significant. Relationships reaching levels of significance $(p \leq 0.20)$ that warrant continued consideration in fully powered studies include HDL and gynoid fat $(r=-0.21 ; p=0.18)$ and coronary risk ratio $(r=0.28 ; p=0.07)$. The correlations range from weak to moderate in strength and negative to positive in direction. As gynoid lean increases, so does coronary risk ratio. HDL decreases as gynoid mass and gynoid lean increases. For relationships reaching levels of significance $(p \leq 0.20)$, as coronary risk ratio increases so does gynoid mass, while HDL decreases as gynoid fat increases.

A significant relationship also exists with triglyceride and visceral fat body lean $(r=0.32 ; p=0.04)$ and coronary risk ratio and visceral fat body lean $(r=0.52 ; p=$ $0.003)$. An inverse relationship exists with HDL and visceral fat body fat $(r=-0.37 ; p=$ $0.01)$, HDL and visceral fat body lean $(r=-0.34 ; p=0.02)$, HDL and visceral fat body mass $(r=0.07 ; p=0.65)$. A positive relationship exists with coronary risk ratio and visceral fat body learn $(\mathrm{r}=0.52 ; \mathrm{p}=0.003)$ and with visceral fat body mass $(\mathrm{r}=0.45 ; \mathrm{p}=$ 0.002; Table 4-16). Relationships reaching levels of significance $(p \leq 0.20)$ that warrant continued consideration in fully powered studies include triglyceride and visceral fat body mass $(r=0.25 ; p=0.10)$, HDL and visceral fat body percent fat $(r=-0.21 ; p=$ $0.17)$, and coronary risk ratio and visceral fat body fat $(r=0.26 ; p=0.09)$. The correlations range from weak to strong in strength and some are negative and some are positive in direction. As visceral fat lean increases, so do triglyceride levels and coronary risk ratio. As HDL increases, visceral fat lean, visceral body fat, and visceral fat mass decrease. For relationships reaching levels of significance $(p \leq 0.20)$, triglyceride and coronary risk ratio goes up, as do visceral fat and visceral fat mass. When visceral body percentage fat goes up, HDL goes down.

An inverse relationship exists with HDL and android gynoid ratio $(r=-0.46 ; p=$ $0.001)$ and with HDL and fat mass ratio $(r=-0.39 ; p=0.007)$, which are presented in Table 4-17. Relationships reaching levels of significance $(p \leq 0.20)$ that warrant continued consideration in fully powered studies include coronary risk ratio and android gynoid ratio $(r=0.23 ; p=0.13)$, Hgb AlC and android percent fat $(r=0.20 ; p=0.19)$, glucose and total fat mass $(r=028 ; p=0.06), \mathrm{Hgb} \mathrm{A1C}$ and total fat mass $(r=0.26 ; p=$ $0.09), \mathrm{HDL}$ and total fat mass $(r=-0.28 ; p=0.06)$, LDL and total fat mass $(r=-0.21 ; p=$ $0.17)$, coronary risk ratio and total fat mass $(r=0.21 ; p=0.16)$, Hgb A1C and fat mass ratio $(r=0.21 ; p=0.17)$, and coronary risk ratio and fat mass ratio $(r=0.20 ; p=0.19)$. The correlations range from weak to moderate in strength with some negative and some positive in direction. As HDL increases, android gynoid ratio and fat mass ratio decrease. For relationships reaching levels of significance $(p \leq 0.20)$, as coronary risk ratio, HgbA1C, and glucose increase, so do android gynoid ratio, android percentage of fat, total fat mass, and fat mass ratio. As HDL and LDL increase, total fat mass decreases.

Table 4-18 shows a significant relationship was found with glucose and WB total fat $(r=0.31 ; p=0.04), \mathrm{HgbA} 1 \mathrm{C}$ and WB total fat $(r=0.30 p=0.05)$, coronary risk ratio and WB total lean $(r=0.41 ; p=0.001)$, and coronary risk ratio and WB total mass $(r=$ 
0.39; $p=0.001)$. An inverse significant relationship was found with HDL and WB total lean $(r=-0.45 ; \mathrm{p}=0.002)$ and HDL $(\mathrm{r}=-0.45 ; \mathrm{p}=0.002)$. As glucose and HgbA1C levels increase, so does WB total fat. AsWB total fat goes up, so do HgbA1C and coronary risk ratio levels. The correlations range from moderate to strong in strength and some are negative and some are positive in direction.

Table 4-18 also shows relationships reaching levels of significance $(p \leq 0.20)$ that warrant continued consideration in fully powered studies include creatinine and WB total fat $(\mathrm{r}=-0.20 ; p=0.20)$, HDL and WB total fat $(r=-0.27 ; p=0.07)$, LDL and WB total fat $(r=-0.22 ; p=0.15)$ demonstrate an inverse relationship. A positive relationship was found between coronary risk ratio and WB total fat $(r=0.20 ; p=0.20)$, diastolic blood pressure and WB total mass $(r=0.20 ; p=0.18)$, WB total PFat and glucose $(r=0.20 ; p=$ $0.20)$, WB total PFat and HgbA1C $(r=0.20 ; p=0.20)$, and WB total mass and glucose $(r$ $=0.24 ; p=0.11)$, and WB total mass and $\operatorname{HgbA} 1 \mathrm{C}(r=0.21 ; p=0.16)$. The correlations are weak in strength and some are negative and some are positive in direction. As creatinine level increases, WB total PFat decreases. As HDL and LDL levels increase, WB total fat decreases. As coronary risk ratio increases, so does WB total fat. As diastolic blood pressure increases, so does WB total mass. WB total PFat goes down as glucose and HgbA1C decrease.

\section{Research Question 4}

Research Question 4 asked, To what degree are anthropometric measures obtained at the time of transplant associated with serum levels of glucose, lipids, and creatinine and elevated blood pressure in kidney transplant recipients at 5-8 years postransplant?

Table 4-19 reports the correlational analyses of anthropometric measures of weight and BMI at the time of transplant and cardiac-related outcomes 5-8 years postkidney transplantation. A significant relationship exists with $\mathrm{Hgb}$ A1C and weight $(r=$ $0.29 ; p=0.06)$ and coronary risk ratio and weight $(r=0.33 ; p=0.03)$. An inverse relationship exists with HDL and weight $(r=-0.46 ; p=0.001)$ and between HDL and BMI $(r=-0.34 ; p=0.02)$. An inverse relationship exists with LDL and BMI $(r=-0.34 ; p$ $=0.04)$. Relationships reaching levels of significance $(p \leq 0.20)$ that warrant continued consideration in fully powered studies include glucose and weight $(r=0.27 ; p=0.079)$, LDL and weight $(r=-0.24 ; p=0.13)$, diastolic BP and weight $(r=0.198 ; p=0.190)$, glucose and BMI $(r=0.28 ; p=0.06)$, and HgbA1C and BMI $(r=0.29 ; p=0.06)$. The correlations range from weak to moderate in strength and some are negative and some are positive in direction. As body weight increases so does coronary risk ratio while HDL decreases; as BMI increases LDL decreases. For relationships reaching levels of significance $(p \leq 0.20)$, as HgbA1C and glucose levels increase, so does BMI. As diastolic BP, HgbA1c, and glucose increase, so does body weight. 
Table 4-19. Relationship Among Anthropometric Measures of Weight and BMI at Time of Transplant and Cardiac-Related Outcomes at 5-8 Years Post-Kidney Transplantation

\begin{tabular}{lcc}
\hline Variables & $\begin{array}{c}\text { Weight } \\
\boldsymbol{r}(\boldsymbol{p} \text { value }) *\end{array}$ & $\begin{array}{c}\text { BMI } \\
\boldsymbol{r}(\boldsymbol{p} \text { value })\end{array}$ \\
\hline Creatinine & $0.002(1.00)$ & $-0.04(0.78)$ \\
Glucose & $0.27(0.08)$ & $0.28(0.06)$ \\
Hgb A1C & $0.30(0.06)$ & $0.29(0.06)$ \\
Triglyceride & $0.12(0.45)$ & $0.15(0.32)$ \\
HDL & $-0.46(0.001)$ & $-0.34(0.02)$ \\
LDL & $-0.24(0.13)$ & $-0.34(0.03)$ \\
Coronary risk ratio & $0.33(0.031)$ & $0.19(0.21)$ \\
Systolic BP & $0.11(0.49)$ & $0.01(0.93)$ \\
Diastolic BP & $0.20(0.19)$ & $0.13(0.38)$ \\
\hline
\end{tabular}

Note. *Pearson Correlation Coefficients: Prob $>[\mathrm{r}]$ under Ho: Rho $=0 ; \mathrm{Hgb}=$ hemoglobin; $\mathrm{HDL}=$ high density lipoprotein; $\mathrm{LDL}=$ low density lipoprotein; $\mathrm{BP}=$ blood pressure; $\mathrm{BMI}=$ body mass index; $r=$ correlation coefficient; $p$ value $\leq 0.05$ and $\leq 0.20$ 


\section{Research Question 5}

Research Question 5 asked, To what degree are changes in anthropometric measures from baseline to 5-8 years posttransplant associated with serum glucose, lipids, and creatinine and elevated blood pressure at 5-8 years posttransplant?

Table 4-20 shows the relationship among weight and BMI change with lab values and elevated blood pressure from baseline to 5-8 years posttransplant. A significant relationship exists with coronary risk ratio and BMI $(r=0.31 ; p=0.04)$. Relationships reaching levels of significance $(p \leq 0.20)$ that warrant continued consideration in fully powered studies include creatinine and BMI $(r=0.25 ; p=0.10)$. The correlations are moderate in strength in a positive in direction. As BMI increases so does coronary risk ratio. For relationships reaching levels of significance $(p \leq 0.20)$, as creatinine increases so does BMI.

\section{Research Question 6}

Research Question 6 asked, To what degree are changes in body composition measures from baseline to 5-8 years posttransplant associated with serum glucose, lipids, and creatinine and elevated blood pressure at 5-8 years posttransplant? Tables 4-21 to 4-26 show the changes in body composition measures from baseline to 5-8 years associated with serum glucose, lipids, and elevated blood pressure at a prolonged time posttransplant.

An inverse relationship was found with creatinine and total fat $(r=-0.35 ; p=$ $0.02)$ and LDL and total lean $(r=-0.37 ; p=0.01)$. The relationship between current LDL and total percent fat $(r=0.32 p=0.05)$ was statistically significant (Table 4-21). Relationships reaching levels of significance $(p \leq 0.20)$ that warrant continued consideration in fully powered studies include creatinine and total mass $(r=-0.28 ; p=$ $0.07)$, creatinine and total percent fat $(r=-0.28 ; p=0.07)$, triglyceride and total lean $(r=$ $-0.26 ; p=0.09)$, coronary risk ratio and total lean $(r=-0.20 ; p=0.20)$, systolic $\mathrm{BP}$ and total fat $(r=-0.21 ; p=0.18)$, diastolic BP and total fat $(r=0.28 ; p=0.07)$, diastolic BP and total mass $(r=0.28 ; p=0.07)$, and diastolic BP and total percent fat $(r=0.27 ; p$ $=0.07)$. The correlations are moderate in strength with some negative and some are positive in direction. As LDL increases so does total percentage of fat. Creatinine and LDL increase while total lean and total fat decrease. For relationships reaching levels of significance $(p \leq 0.20)$, ascreatinine increases, total mass decrease. As creatinine decreases, so does total percet of fat. As triglycerides and coronary risk ratio increase, total lean decreases. As systolic blood pressure increase, total fat decreases, and total mass, total lean, and total percentage of fat decrease. As total percent fat, total fat, and total mass increase, so does diabstolic BP. The correlations are moderate in strength with a positive direction. 
Table 4-20. Relationship Among Cardiac-Related Outcomes at 5-8 Years PostKidney Transplantation and Change in Anthropometric Measures of Weight and BMI from Time of Transplant to 5-8 Years Post-Kidney Transplantation

\begin{tabular}{lcc}
\hline Variables & $\begin{array}{c}\text { Weight } \\
\boldsymbol{r}(\boldsymbol{p} \text { value })^{*}\end{array}$ & $\begin{array}{c}\text { BMI } \\
\boldsymbol{r}(\boldsymbol{p} \text { value)* }\end{array}$ \\
\hline Creatinine & $0.02(0.87)$ & $0.25(0.10)$ \\
Glucose & $-0.17(0.27)$ & $-0.05(0.77)$ \\
Hgb A1C & $-0.09(0.56)$ & $-0.03(0.83)$ \\
Triglyceride & $-0.06(0.69)$ & $0.03(0.86)$ \\
HDL & $0.08(0.60)$ & $-0.15(0.32)$ \\
LDL & $-0.01(0.93)$ & $0.07(0.64)$ \\
Coronary risk ratio & $0.01(0.95)$ & $0.31(0.04)$ \\
Systolic BP & $-0.03(0.85)$ & $0.05(0.73)$ \\
Diastolic BP & $0.15(0.34)$ & $0.18(0.24)$ \\
\hline
\end{tabular}

Note: *Pearson Correlation Coefficients: Prob $>[\mathrm{r}]$ under Ho: Rho $=0$; Hgb $=$ hemoglobin; $\mathrm{HDL}=$ high density lipoprotein; $\mathrm{LDL}=$ low density lipoprotein; $\mathrm{BP}=$ blood pressure; $\mathrm{BMI}=$ body mass index; $r=$ correlation coefficient; $p$ value $\leq 0.05$ and $\leq 0.20$.

Table 4-21. Relationship Among Cardiac-Related Outcomes at 5-8 years PostKidney Transplantation and Change in Body Composition Measures of Total Fat, Total Lean, Total Mass, and Total PFat from Time of Transplant to 5-8 Years PostKidney Transplantation

\begin{tabular}{lcccc}
\hline Variables & $\begin{array}{c}\text { Total Fat } \\
\boldsymbol{r}(\boldsymbol{p} \text { value })^{*}\end{array}$ & $\begin{array}{c}\text { Total Lean } \\
\boldsymbol{r}(\boldsymbol{p} \text { value })^{*}\end{array}$ & $\begin{array}{c}\text { Total Mass } \\
\boldsymbol{r}(\boldsymbol{p} \text { value })^{*}\end{array}$ & $\begin{array}{c}\text { Total PFat } \\
\boldsymbol{r}(\boldsymbol{p} \text { value })^{*}\end{array}$ \\
\hline Creatinine & $-0.35(0.02)$ & $-0.07(0.64$ & $-0.28(0.065)$ & $-0.28(0.07)$ \\
Glucose & $-0.02(0.91)$ & $-0.02(0.90)$ & $-0.021(0.90)$ & $0.01(0.93)$ \\
HgbA1C & $0.06(0.68)$ & $0.10(0.50)$ & $0.095(0.54)$ & $0.01(1.00)$ \\
Triglyceride & $-0.04(0.81)$ & $-0.26(0.09)$ & $-0.153(0.32)$ & $0.07(0.66)$ \\
HDL & $-0.02(0.89)$ & $0.00(0.69)$ & $0.02(0.89)$ & $-0.02(0.89)$ \\
LDL & $0.08(0.62)$ & $-0.37(0.01)$ & $-0.12(0.44)$ & $0.32(0.05)$ \\
Coronary risk & $0.08(0.62)$ & $-0.20(0.20)$ & $-0.04(0.79)$ & $0.16(0.31)$ \\
ratio & & & & \\
Systolic BP & $-0.21(0.18)$ & $0.03(0.85)$ & $-0.13(0.38)$ & $-0.17(0.25)$ \\
Diastolic BP & $0.28(0.07)$ & $0.16(0.30)$ & $0.28(0.07)$ & $0.27(0.07)$ \\
\hline
\end{tabular}

Note: *Pearson Correlation Coefficients: Prob $>[\mathrm{r}]$ under Ho: Rho $=0$; Hgb $=$ hemoglobin; $\mathrm{HDL}=$ high density lipoprotein; $\mathrm{LDL}=$ low density lipoprotein; $\mathrm{PFat}=$ percent fat; $r=$ correlation coefficient; $p$ value $\leq 0.05$ and $\leq 0.20$. 
Table 4-22. Relationship Among Cardiac Related Outcomes at 5-8 Years PostKidney Transplant and Changes in Body Composition Measures of Android Fat, Android Lean, Android Mass, and Android PF from Time of Transplant to 5-8 Years Post-Kidney Transplantation

\begin{tabular}{lcccc}
\hline Variables & $\begin{array}{c}\text { Android Fat } \\
\boldsymbol{r}(\boldsymbol{p} \text { value })\end{array}$ & $\begin{array}{c}\text { Android Lean } \\
\boldsymbol{r}(\boldsymbol{p} \text { value })^{*}\end{array}$ & $\begin{array}{c}\text { Android } \\
\text { Mass } \\
\boldsymbol{r}(\boldsymbol{p} \text { value }) *\end{array}$ & $\begin{array}{c}\text { Android } \\
\text { PFat } \\
\boldsymbol{r}(\boldsymbol{p} \text { value }) *\end{array}$ \\
\hline Creatinine & $-0.34(0.02)$ & $0.08(0.60)$ & $-0.22(0.14)$ & $-0.37(0.01)$ \\
Glucose & $0.00(0.99)$ & $0.04(0.80)$ & $0.02(0.900)$ & $0.01(0.96)$ \\
Hgb A1C & $0.08(0.60)$ & $0.11(0.48)$ & $0.11(0.47)$ & $0.00(0.89)$ \\
Triglyceride & $0.11(0.46)$ & $-0.44(0.002)$ & $-0.13(0.41)$ & $0.17(0.26)$ \\
HDL & $-0.01(0.93)$ & $0.06(0.69)$ & $0.027(0.91)$ & $0.02(0.89)$ \\
LDL & $-0.00(0.90)$ & $-0.32(0.41)$ & $-0.16(0.31)$ & $0.22(0.16)$ \\
Coronary risk & $0.08(0.63)$ & $-0.16(0.29)$ & $-0.02(0.92)$ & $0.11(0.47)$ \\
ratio & & & & \\
Systolic BP & $-0.24(0.11)$ & $-0.12(0.43)$ & $-0.13(0.40)$ & $-0.25(0.09)$ \\
Diastolic BP & $0.30(0.05)$ & $0.23(0.13)$ & $0.33(0.03)$ & $0.26(0.08)$ \\
\hline
\end{tabular}

Note: *Pearson Correlation Coefficients: Prob $>[\mathrm{r}]$ under Ho: Rho=0; Hgb $=$

hemoglobin; HDL = high density lipoprotein; $\mathrm{LDL}=$ low density lipoprotein; $\mathrm{BP}=$ blood pressure; PFat $=$ percent fat; $r=$ correlation coefficient; $p$ value $\leq 0.05$ and $\leq 0.20$. 
Table 4-23. Relationship Among Cardiac-Related Outcomes at 5-8 Years PostKidney Transplantation and Changes in Body Composition Measures of Gynoid Fat, Gynoid Lean, Gynoid Mass, and Gynoid PFat from Time of Transplant to 5-8 Years Post-Kidney Transplantation

\begin{tabular}{lcccc}
\hline Variables & $\begin{array}{c}\text { Gynoid Fat } \\
\boldsymbol{r}(\boldsymbol{p} \text { value }) *\end{array}$ & $\begin{array}{c}\text { Gynoid Lean } \\
\boldsymbol{r}(\boldsymbol{p} \text { value })^{*}\end{array}$ & $\begin{array}{c}\text { Gynoid Mass } \\
\boldsymbol{r}(\boldsymbol{p} \text { value })^{*}\end{array}$ & $\begin{array}{c}\text { Gynoid PFat } \\
\boldsymbol{r}(\boldsymbol{p} \text { value })^{*}\end{array}$ \\
\hline Creatinine & $-0.33(0.03)$ & $-0.18(0.25)$ & $-0.31(0.04)$ & $-0.20(0.21)$ \\
Glucose & $-0.03(0.83)$ & $-0.06(0.70)$ & $-0.05(0.73)$ & $0.01(0.93)$ \\
Hgb A1C & $0.04(0.78)$ & $0.09(0.54)$ & $0.08(0.61)$ & $-0.01(0.97)$ \\
Triglyceride & $-0.01(0.68)$ & $-0.26(0.08)$ & $-0.11(0.24)$ & $0.06(0.70)$ \\
HDL & $-0.03(0.86)$ & $0.08(0.60)$ & $0.02(0.87)$ & $-0.06(0.72)$ \\
LDL & $0.17(0.30)$ & $-0.38(0.01)$ & $-0.08(0.60)$ & $0.37(0.01)$ \\
Coronary risk ratio & $0.07(0.64)$ & $-0.20(0.18)$ & $-0.06(0.71)$ & $0.18(0.25)$ \\
Sytolic BP & $-0.16(0.29)$ & $-0.04(0.80)$ & $-0.13(0.40)$ & $-0.10(0.50)$ \\
Diastolic BP & $0.25(0.10)$ & $0.10(0.51)$ & $0.22(0.14)$ & $0.26(0.10)$ \\
\hline
\end{tabular}

Note: *Pearson Correlation Coefficients: Prob $>$ [r] under Ho: Rho $=0$;

$\mathrm{Hgb}=$ hemoglobin; HDL = high density lipoprotein; LDL = low density lipoprotein;

PFat $=$ percent fat; $r=$ correlation coefficient; $p$ value $\leq 0.05$ and $\leq 0.20$. 
Table 4-24. Relationship Among Cardiac-Related Outcomes at 5-8 Years PostKidney Transplantation and Changes in Body Composition Measures of VFat Body Fat, VFat Body Lean, VFat Body Mass, and VFat Body PFat from Time of Transplant to 5-8 Years Post-Kidney Transplantation

\begin{tabular}{|c|c|c|c|c|}
\hline Variables & $\begin{array}{c}\text { VFat Body } \\
\text { Fat } \\
r(p \text { value }) * \\
\end{array}$ & $\begin{array}{c}\text { VFat Body } \\
\text { Lean } \\
r(p \text { value }) *\end{array}$ & $\begin{array}{c}\text { VFat Body } \\
\text { Mass } \\
r(p \text { value }) * \\
\end{array}$ & $\begin{array}{c}\text { VFat Body } \\
\text { PFat } \\
r(p \text { value }) *\end{array}$ \\
\hline Creatinine & $-0.36(0.01)$ & $0.14(0.38)$ & $-0.27(0.08)$ & $-0.36(0.02)$ \\
\hline Glucose & $0.03(0.84)$ & $0.14(0.36)$ & $0.09(0.58)$ & $0.01(0.93)$ \\
\hline Hgb A1C & $0.08(0.57)$ & $0.21(0.18)$ & $0.16(0.29)$ & $0.02(0.89)$ \\
\hline Triglyceride & $-0.01(1.00)$ & $-0.30(0.05)$ & $-0.13(0.41)$ & $0.06(0.71)$ \\
\hline HDL & $-0.02(0.89)$ & $-0.01(0.96)$ & $-0.02(0.88)$ & $0.02(0.88)$ \\
\hline LDL & $0.05(0.77)$ & $-0.34(0.03)$ & $-0.09(0.56)$ & $0.23(0.15)$ \\
\hline Coronary risk ratio & $0.07(0.67)$ & $-0.26(0.09)$ & $-0.05(0.77)$ & $0.11(0.50)$ \\
\hline Systolic BP & $-0.27(0.07)$ & $0.07(0.62)$ & $-0.21(0.16)$ & $-0.25(0.09)$ \\
\hline Diastolic BP & $0.27(0.07)$ & $0.13(0.41)$ & $0.29(0.05)$ & $0.27(0.08)$ \\
\hline
\end{tabular}

Note: *Pearson Correlation Coefficients: Prob $>[\mathrm{r}]$ under Ho: Rho $=0 ; \mathrm{Hgb}=$ hemoglobin; HDL = high density lipoprotein; LDL = low density lipoprotein; $\mathrm{BP}=$ blood pressure; VFat $=$ visceral fat; PFat $=$ percent fat; $r=$ correlation coefficient; $p$ value $\leq 0.05$ and $\leq 0.20$. 
Table 4-25. Relationship Among Cardiac-Related Outcomes at 5-8 Years PostKidney Transplantation and Changes in Body Composition Measures of Android Gynoid Ratio, Android Percent Fat, Total Fat Mass, and Fat Mass Ratio from Time of Transplant to 5-8 Years Post-Kidney Transplantation

\begin{tabular}{lcccc}
\hline Variables & $\begin{array}{c}\text { Android } \\
\text { Gynoid Ratio } \\
\boldsymbol{r}(\boldsymbol{p} \text { value)* }\end{array}$ & $\begin{array}{c}\text { Android } \\
\text { Percent Fat } \\
\boldsymbol{r}(\boldsymbol{p} \text { value })^{*}\end{array}$ & $\begin{array}{c}\text { Total Fat } \\
\text { Mass } \\
\boldsymbol{r}(\boldsymbol{p} \text { value })^{*}\end{array}$ & $\begin{array}{c}\text { Fat Mass } \\
\text { Ratio } \\
\boldsymbol{r}(\boldsymbol{p} \text { value })^{*}\end{array}$ \\
\hline Creatinine & $-0.41(0.01)$ & $-0.33(0.03)$ & $-0.33(0.03)$ & $-0.40(0.01)$ \\
Glucose & $0.03(0.83)$ & $-0.01(0.93)$ & $-0.00(1.00)$ & $0.13(0.40)$ \\
Hgb A1C & $0.03(0.87)$ & $-0.02(0.89)$ & $0.05(0.75)$ & $0.14(0.36)$ \\
Triglyceride & $0.11(0.50)$ & $0.07(0.65)$ & $0.00(0.99)$ & $0.11(0.50)$ \\
HDL & $0.10(0.53)$ & $-0.01(0.97)$ & $-0.06(0.70)$ & $0.08(0.61)$ \\
LDL & $0.01(0.94)$ & $0.25(0.11)$ & $0.08(0.62)$ & $-0.11(0.50)$ \\
Coronary risk ratio & $0.05(0.72)$ & $0.13(0.37)$ & $0.11(0.46)$ & $0.04(0.80)$ \\
Systolic BP & $-0.40(0.01)$ & $-0.27(0.07)$ & $-0.26(0.08)$ & $-0.24(0.11)$ \\
Diastolic BP & $0.07(0.63)$ & $0.17(0.25)$ & $0.18(0.22)$ & $0.21(0.17)$ \\
\hline
\end{tabular}

Note: *Pearson Correlation Coefficients: Prob $>$ [r] under Ho: Rho=0;

$\mathrm{Hgb}=$ hemoglobin; HDL = high density lipoprotein; LDL = low density lipoprotein; BP $=$ blood pressure; $r=$ correlation coefficient; $p$ value $\leq 0.05$ and $\leq 0.20$. 
Table 4-26. Relationship Among Cardiac-Related Outcomes at 5-8 Years PostKidney Transplantation and Change in Body Composition Measures of WBTotal Fat, WBTotal Lean, WBTotal Mass, and WBTotal PFat from Time of Transplant to 5-8 Years Post-Kidney Transplantation

\begin{tabular}{|c|c|c|c|c|}
\hline Variables & $\begin{array}{l}\text { WB Total } \\
\text { Fat } \\
r(p \text { value })\end{array}$ & $\begin{array}{l}\text { WB Total } \\
\text { Lean } \\
r(p \text { value })\end{array}$ & $\begin{array}{l}\text { WB Total } \\
\text { Mass } \\
r(p \text { value })\end{array}$ & $\begin{array}{l}\text { WB Total } \\
\text { PFat } \\
r \text { ( } p \text { value })\end{array}$ \\
\hline Creatinine & $-0.14(0.35)$ & $-0.12(0.42)$ & $-0.15(0.33)$ & $-0.06(0.70)$ \\
\hline Glucose & $-0.42(0.004)$ & $-0.16(0.30)$ & $-0.31(0.04)$ & $-0.37(0.01)$ \\
\hline Hgb A1C & $-0.40(0.01)$ & $-0.15(0.35)$ & $-0.29(0.05)$ & $-0.34(0.02)$ \\
\hline Triglyceride & $0.02(0.91)$ & $0.02(0.92)$ & $0.02(0.90)$ & $-0.01(0.93)$ \\
\hline HDL & $0.28(0.06)$ & $0.39(0.01)$ & $0.38(0.01)$ & $0.00(1.0)$ \\
\hline LDL & $0.03(0.87)$ & $-0.01(1.0)$ & $0.01(0.95)$ & $0.01(0.94)$ \\
\hline Coronary risk ratio & $-0.15(0.34)$ & $-0.27(0.08)$ & $-0.24(0.12)$ & $-0.00(1.0)$ \\
\hline Systolic BP & $-0.25(0.09)$ & $-0.40(0.01)$ & $-0.37(0.01)$ & $-0.04(0.80)$ \\
\hline Diastolic BP & $-0.27(0.07)$ & $-0.27(0.07)$ & $-0.30(0.05)$ & $-0.14(0.37)$ \\
\hline
\end{tabular}

Note. *Pearson Correlation Coefficients: Prob $>$ [r] under Ho: Rho $=0 \mathrm{WB}=$ whole-body; $\mathrm{Hgb}=$ hemoglobin; HDL = high density lipoprotein; LDL = low density lipoprotein; BP $=$ blood pressure; PFat $=$ percent fat; $r=$ correlation coefficient; $p$ value $\leq 0.05$ and $\leq$ 0.20 . 
An inverse relationship was found between creatinine and android fat $(r=-0.34 ; p$ $=0.02)$, creatinine and android percent fat $(r=-0.366 ; p=0.014)$, and triglyceride and android lean $(r=-0.44 ; p=0.002)$. Diastolic BP and android fat $(r=0.29 ; p=0.05)$ and diastolic BP and android mass $(r=0.33 ; p=0.03)$ are shown in Table 4-22. Relationships reaching levels of significance $(p \leq 0.20)$ that warrant continued consideration in fully powered studies include creatinine and android mass $(r=-0.22 ; p=$ $0.14)$, LDL and android percent fat $(r=0.22 ; p=0.16)$, systolic and android fat $(r=-$ $0.24 ; p=0.11)$, systolic BP and android percent fat $(r=-0.25 ; p=0.09)$, diastolic BP and android lean $(r=0.23 ; p=0.13)$, and diastolic BP and android percent fat $(r=0.26 ; p=$ $0.08)$. As creatinine increases, android fat, android mass, and android percentage of fat decrease. While triglycerides increase, android lean decreases. As diastolic blood pressure increases so do android fat and android mass. These correlations were weak to moderate in strength. For relationships reaching levels of significance $(p \leq 0.20)$, systolic blood pressure increases as android fat and android percentage of fat decrease. As creatinine increases, android mass decreases; while LDL increases so does android percent of fat. These correlations are considered weak to strong in strength and are positive in direction (Sheskin, 2011).

Table 4-23 indicates a significant relationship was found with LDL and gynoid percent fat $(r=0.37 ; p=0.01)$. An inverse relationship was found with creatinine and gynoid fat $(r=-0.33 ; \mathrm{p}=0.03)$, creatinine and gynoid mass $(r=-0.31 ; p=0.04)$, and LDL and gynoid lean $(r=-0.38 ; p=0.01)$. Relationships reaching levels of significance $(p \leq 0.20)$ that warrant continued consideration in fully powered studies include triglyceride and gynoid lean $(r=-0.26 ; p=0.08)$, coronary risk ratio $(r=-0.20 ; p=0.18)$, diastolic and gynoid fat $(r=0.249 ; p=0.10)$, diastolic and gynoid mass $(r=0.22 ; p=$ $0.14)$, and diastolic and gynoid percent fat $(r=0.26 ; p=0.08)$. As LDL increases so does gynoid percentage of fat. While creatinine increases, gynoid fat and gynoid mass decrease; while LDL increases gynoid lean decreases. These correlations were moderate in strength. For relationships reaching levels of significance $(p \leq 0.20)$, triglyceride increases while gynoid lean and coronary risk ratio decreases. As diastolic blood pressure increases so do gynoid fat, gynoid mass, and gynoid percentage of fat. These correlations are weak in strength, and some are negative and some positive (Sheskin, 2011).

An inverse relationship with significance exists with creatinine and visceral fat body fat $(r=-0.36 ; p=0.01)$, creatinine and visceral fat body percent fat $(r=-0.36 ; p=$ $0.02)$, triglyceride and visceral fat body lean $(r=-0.30 ; p=0.05)$, and with LDL and visceral fat body lean $(r=-0.34 ; p=0.03)$. The diastolic BP and visceral fat body mass $(r$ $=0.29 ; p=0.05$ ) are statistically significant (Table 4-24). Relationships reaching levels of significance $(p \leq 0.20)$ that warrant continued consideration in fully powered studies include creatinine and visceral fat body mass $(r=-0.27 ; p=0.08)$, Hgb A1C and visceral fat body lean $(r=0.21 ; p=0.18)$, LDL and visceral fat body percent fat $(r=0.23 ; p=$ $0.15)$, coronary risk ratio and visceral fat body lean $(r=-0.26 ; p=0.09)$, systolic and visceral fat body fat $(r=-0.27 ; p=0.07)$, systolic BP and visceral fat body mass $(r=-$ $0.21 ; p=0.16)$, systolic BP and visceral fat body percent fat $(r=-0.25 ; p=0.09)$, diastolic BP and visceral fat body fat $(r=0.27 ; p=0.07)$, and diastolic BP and visceral fat body percent fat $(r=0.27 ; p=0.08)$. As creatinine increases, visceral fat body and 
visceral body percentage of fat decrease. Triglyceride and LDL increase while visceral fat body lean decreases. As diastolic BP increases so does visceral fat body mass. These correlations were weak to moderate in strength. For relationships reaching levels of significance $(p \leq 0.20)$, as creatinine and systolic BP go up visceral fat body mass decreases. As HgbAIC goes up and coronary risk ratio goes down, visceral fat body lean increases. As LDL and diastolic BP increases, so does visceral fat body percentage. As systolic BP goes up , visceral fat body mass goes down. These correlations are considered weak in strength; some are negative and some positive (Sheskin, 2011).

Table 4-25 indicates a relationship was found with creatinine and android gynoid ratio $(r=-0.41 ; p=0.01)$, creatinine and android percent fat $(r=-0.33 ; p=0.03)$, creatinine and total fat mass $(r=-0.33 ; p=0.03)$, and creatinine and fat mass ratio $(r=-$ $0.40 ; p=0.01$ ) and were all inversely related. An inverse relationship was found with systolic BP and android gynoid ratio $(r=-0.40 ; p=0.01$; Table 4-25). Relationships reaching levels of significance $(p \leq 0.20)$ that warrant continued consideration in fully powered studies include LDL and android percent fat $(r=0.25 ; p=0.11)$,systolic BP and android/gynoid ratio $(r=-0.40 ; p=0.001)$ systolic BP and total fat mass $(r=-0.26 ; p=$ $0.08)$, systolic BP and fat mass ratio $(r=-0.24 ; p=0.11)$, and diastolic BP and fat mass ratio $(r=0.21 ; p=0.17)$. As creatinine goes up, android gynoid ratio, android percent of fat, total fat mass, and fat mass ratio go down. As systolic BP increases, android gynoid ratio decreases. These correlations were moderate to strong in strength. For relationships reaching levels of significance $(p \leq 0.20)$, as LDL increases so does android percent of fat. As diastolic BP increases, so does fat mass ratio. While systolic blood pressure goes up, android gynoid ratio, android percentage of fat, and total fat mass go down. These correlations are considered to range from weak to strong in strength with some negative and some positive (Sheskin, 2011).

Table 4-26 indicates an inverse relationship with glucose and WB total fat $(r=-$ $0.42 ; p=0.004)$, WB total mass $(r=-0.31 ; p=0.04)$, and WB total PFat $(r=-0.37 ; p=$ $0.01)$ and with HgbA1C and WB total fat $(r=-0.40 ; p=0.01)$, WB total mass $(r=-$ $0.29 ; \mathrm{p}=0.05)$, and WB total PFat $(r=-0.34 ; p=0.02)$, with systolic BP and WB total lean $(r=-0.40 ; p=0.01)$ and WB total mass $(r=-0.37 ; p=0.01)$, with diastolic BP and WB total mass $(r=-0.30 ; p=0.05)$. A significant relationship was found with HDL and WB total lean $(r=0.39 ; p=0.01)$ and HDL and WB total mass $(r=0.38 ; p=0.01)$. The correlations were weak to strong and some are negative and some positive in direction. As glucose goes up, so do WB total fat, WB total mass, and WB total PFat. As systolic BP goes down, WB total lean and WB total mass go up. As diastolic BP goes down, WB total mass goes up. As HDL goes up, so do WB total lean and WB total mass. As glucose and HgbA1C levels go up, WB total fat goes down. As WB total lean and WB total fat go up, HgbA1C and coronary risk ratio levels go down.

Relationships reaching levels of significance $(p \leq 0.20)$ that warrant continued consideration in fully powered studies with an inverse relationship include HDL and WB total fat $(r=0.28 ; p=0.06)$, coronary risk ratio and WB total lean $(r=-0.27 ; p=0.08)$, coronary risk ratio and $\mathrm{WB}$ total mass $(r=-0.24 ; p=0.12)$, diastolic $\mathrm{BP}$ and $\mathrm{WB}$ total fat $(r=-0.27 ; p=0.07)$, diastolic BP and WB total lean $(\mathrm{r}=-0.27 ; p=0.07)$, and systolic BP 
and WB total fat $(\mathrm{r}=-0.25 ; p=0.09)$. The correlations were weak in relationship and some are negative and some positive in direction. As coronary risk ratio increases, WB total lean and WB total mass decrease. As diastolic BP increases so do WB total fat and WB total lean. As HDL level increases, so does WB total fat. 


\section{CHAPTER 5. DISCUSSION, CONCLUSION, AND RECOMMENDATIONS}

Previous studies have reported significant weight gain (between 6 to $10 \mathrm{~kg}$ ) in patients the first year post-kidney transplantation (Aksoy, 2016; Cashion et al., 2014). Weight gain following transplantation is known to increase obesity risk factors and contribute to comorbid disorders such as HTN Type II diabetes and CVD, which is the number one cause of mortality in this population (Beckmann, Ivanović, Drent, Ruppar, \& Geest, 2015; Olarte \& Hawasli, 2009). Despite the high percentage of individuals gaining a significant amount of weight following transplantation and the serious consequences associated with this weight gain, no studies have examined whether weight distribution throughout the body is associated with cardiac risk factors.

Moreover, while concern exists for the increased cardiac risk that accompanies post-kidney transplant weight gain; in addition to the differential risks that may be associated with weight gain distribution, there is a lack of consensus regarding what measures of body weight provide the best information on these cardiac risk factors. Typical anthropometric measures of weight often include BMI and waist circumference. However, measures of body mass by DXA provide more precise data of lean and fat mass for various compartments of the body, and visceral fat in particular, which is believed to be associated with increased cardiac risk. Thus, the present study sought to address whether anthropometric or DXA measures of weight are most closed associated with cardiac risk factors. This chapter includes a discussion of the significant findings and compares the study results with existing research. Furthermore, it includes strengths and limitations of our study, practice implications, theoretical implications, as well as recommendations for future research and a conclusion.

\section{Demographic Findings}

Unlike national data, the majority of our study sample (64.4\%) was African American and reflective of the demographic characteristics of the community from which the sample was drawn. Moreover, our study cohort also captured a group at particularly high risk for cardiovascular disease in general and graft failure following kidney transplant in particular. Our findings, therefore, provide new information particularly relevant to this high-risk population.

\section{Discussion}

\section{Research Question 1}

The first research question sought to determine the association between anthropometric measures and serum glucose, lipid, and creatinine levels and blood pressure in kidney transplant recipients 5-8 years posttransplant. The study found that 
high waist readings (WC and waist-to-hip ratio) were associated with an increased coronary risk ratio $(r=0.33 ; p=0.05)$, and that higher diastolic BPs (above $90 \mathrm{mmhg}$ ) were associated with higher posttransplant weight. These findings are consistent with previous studies that reported posttransplant weight gain and found that it leads to a high diastolic BP, which in turn increases the renal recipient's risk of developing CVD (Mohammadifard et al., 2013; Scherrer et al., 1991) and acute graft loss (Cosio et al., 2001). Similarly, a high WC also increases the coronary risk ratio, which in turn increases the kidney recipient risk of developing CVD (Mohammadifard et al., 2013).

\section{Research Question 2}

Research Question 2 sought to address the association between body composition measures and serum glucose, lipid, and creatinine levels and blood pressure in kidney transplant recipients 5-8 years posttransplant. While Research Question 1 found increased WC and weight to be associated with increased CV risk and diastolic BP, respectively, several additional associations were found in Research Question 2 results between serum glucose, lipid, and creatinine levels and elevated blood pressure and measures of body composition. In addition to $\mathrm{CV}$ risk, measures of body composition were also associated with HDL, systolic BP, and creatinine. It was also found that android fat, visceral fat, and visceral fat body mass were all found to be associated with increased CV risk (Table 4-8 to 4-11).

Furthermore, using measures of body composition, relationships with HDL were found with measures of total, total PFat, VFat, gynoid PFat, android PFat, and VFat body PFat and with percent gynoid and android fat (Table 4-7 to 4-11). Because approximately one third of recipients undergoing kidney transplant are significantly burdened with CAD and many experience subsequent CV events (Neale \& Smith, 2015), measures of cardiac risk warrant careful monitoring.

Instead of the relationships that Research Question 1 results found between weight and diastolic BP, using measures of body composition, relationships were found with the more meaningful measure of systolic BP in Research Question 2. Specifically, measures of visceral percent fat and android fat were found to be associated with systolic BP. However, surprisingly, these were inverse relationships, thus indicating that increased fat in these compartments was associated with decreased systolic BP. Last, using measures of body composition, additional relationships were found with creatinine levels. Total fat, android Pfat, gynoid Pfat, VFat, and VFat body PFat were all found to be inversely related to creatinine-that is, as these levels increased, creatinine levels decreased, which indicates improved function (Tables 4-7 to 4-12). This-result suggests that elevated levels of total fat, total percent fat, visceral fat, android fat, and gynoid fat are associated with low creatinine level, which reduces the risk of graft loss. However, a high level of creatinine, according to Younespour et al. (2016) and Maraghi et al. (2016), increases graft loss as well as the survivability of the kidney. An inverse relationship exists with creatinine and android percent fat $(r=-0.45 ; p=0.001)$, implying a high android percent fat lowers creatinine level. 


\section{Research Question 3}

Research Question 3 sought to determine the degree to which body composition measures obtained from recipients at the time of transplant were associated with serum levels of glucose, lipids, and creatinine and elevated blood pressure obtained at 5-8 years following the transplant. Significant relationships were found among 11 measures of body composition obtained at the time of transplantation and the HDL level at 5-8 years posttransplant (Tables 4-13 to 4-18). These measures included both android and gynoid lean and mass, as well as the android/gynoid ratio, percent body lean and total lean, percent body fat, fat mass ratio, and total fat and total mass. Because HDL levels at the time of transplant were not available, it was not possible to determine whether this association also existed at the time of transplantation. Regardless, it is noteworthy that all four measures of lean tissue as well as nearly half the remaining compartments measured at the time of transplant surgery were found to be related to HDL levels 5-8 years later. In terms of coronary risk ratio, three of the four measures of lean tissue (total, gynoid, and visceral fat body) were related, along with android fat and mass and total percent fat. However, total fat and visceral fat body lean were related in an unexpected directioni.e., as lean values increased, so did coronary risk. Lastly, lean tissue android and visceral fat body were also related to triglyceride levels. The prevalence of lean tissue relationships to measure cardiac health raises a question regarding the perception that fat mass is of primary concern while increased levels of lean are considered healthier.

In addition, HDL is used to assess patients' risk for heart disease (Hewing, Moore, \& Fisher, 2012). An inverse relationship was found with HDL and total fat ( $r=$ 0.34; $p=0.02)$ and HDL and total lean $(r=-0.50 ; p=<0.001)$, which suggests a high total fat, total lean, and total mass lowers serum levels of HDL. Low serum levels of HDL from a high level of total fat, total lean, and total mass can increase the post-kidney transplant recipients' risk for CAD, which is corroborated by the Framingham study (Castelli et al., 1986). The Framingham study showed the risk for CAD increases as triglyceride, total cholesterol, and LDL increase and those patients with CAD had low levels of HDL (Castelli et al., 1977; Castelli et al., 1986). In addition, Toth (2004) indicated that low serum levels of HDL are often seen with patients who have CAD. Low serum levels of HDL are also an independent risk factor for CAD (Puri et al., 2014; Toth, 2004).

An inverse relationship was found with HDL and visceral body fat $(\mathrm{r}=-0.46 ; p=$ $0.001)$, HDL and visceral body lean $(n=45) ;(r=-0.341 ; p=0.021)$, and HDL and visceral fat body mass $(r=0.07 ; p=0.65)$. This indicates that a high visceral body fat mass and visceral body lean, lowers serum levels of HDL, which predisposes kidney recipients to obesity-related health complications. An elevated level of visceral fat in the abdominal area is linked to health complications such as Type 2 diabetes and CAD (Purnell et al., 2000). 
An inverse relationship exists with HDL and android fat $(r=-0.46 ; p=0.001)$, HDL and android lean $(r=-0.45 ; p=0.002)$, and HDL and android mass $(r=-0.53 ; p<$ 0.001). Android lean is associated with lower serum levels of HDL, indicating the participants in this study were at risk for CVD. This finding is surprising because one would expect that participants with a high android lean would raise the serum levels of HDL, which would lower the participants' risk for CVD rather than increase their risk. Our study found that a high android mass lowers the serum levels of HDL, which is consistent with Latt et al.'s (2018) finding, which showed that a high android mass worsens serum levels of HDL, leading to an increased risk for CVD in normal weight and overweight boys during puberty. It is the overall mass in the abdomen that is the issue whether lean or fat.

High android fat is associated with lower serum levels of HDL, indicating the participants in the current study are at risk for CVD. Android fat distribution is associated with CVD (Kouda, Nakamura, Fujita, Ohara, \& Iki, 2012). Min and Min (2014) studied 5,696 adults from 2003 to 2006 to determine which fat percentages from android and/or gynoid fat predicted CVD. Their findings showed that regardless of gender, android and gynoid body fat percentages are positively linked to BMI and WC. Moreover, increases in android fat percentage were positively linked to total cholesterol, triglycerides, and HDL for males and total cholesterol, HDL, and LDL for females (Min \& Min, 2014). On the other hand, gynoid fat percentage was positively linked to total cholesterol in males. For females, gynoid fat percentages were positively linked to triglycerides and HDL. Min and Min (2014) concluded that fat accumulation in the android region was a significant predictor of CVD compared to that in the gynoid region.

A significant relationship was found with triglyceride and android lean; $(r=0.30$; $p=0.05)$, which implies that low levels of android lean are associated with lower levels of triglycerides. Android lean represents the lower body fat. Jensen (2008) posited a decreased level of lower body fats in the android region decreases patients' risk of CVD. An inverse relationship exists with HDL and gynoid lean $(r=-0.51 ; p<0.001)$. This suggests a high gynoid lean lowers the level of HDL, which is surprising because one would expect that lean body mass in the gynoid region would increase the levels of HDL rather than decrease them.

A significant relationship exists with coronary risk ratio and total lean $(r=0.42 ; p$ $=0.004)$, implying that a high total lean leads to a high coronary risk ratio. Huang et al. (2015) found that high lean mass is an independent factor that increased the survival rates of Chinese patients with CAD because it protected them from mortality. These findings were similar to the findings of Han et al. (2010) and Lavie et al. (2011) in showing that high lean mass is an independent risk factor that increases the survival rates of elderly patients and patients with CAD. The relationship between coronary risk ratio and total mass $(r=0.38 ; p=0.01)$ were significant, suggesting a high total percent fat leads to a high coronary risk ratio.

The relationship between coronary risk ratio and visceral fat body lean $(r=0.52$; $p=0.003$ ) was significant, meaning a lower visceral fat lowers the coronary risk ratio, 
which supports previous findings. Ross and Janiszewski (2008) found weight loss, particularly in the abdominal area, reduces abdominal fat, which in return reduces the patients' risk of CVD. The relationship between coronary risk ratio and android fat $(r=$ $0.32 ; p=0.03)$ and coronary risk ratio and android mass $(r=0.47 ; p=0.001)$ was significant. This indicates that a high android fat and a high android mass increase the coronary risk ratio. Despres (2012) found that regional body fat distribution, particularly android fat and android mass, increases the patients' risk of having a CV event. A significant relationship exists with coronary risk ratio and gynoid lean $(r=0.36 ; p=$ 0.02 ), implying that lean body mass lowers gynoid fat, which reduces the coronary risk ratio; this is consistent with previous findings showing how lowering gynoid fat by replacing it with lean body mass can improve a patient's CV outcome.

\section{Research Question 4}

Research Question 4 sought to determine whether anthropometric measures obtained at the time of transplant were associated with serum levels of glucose, lipids, and creatinine and blood pressure in kidney transplant recipients at 5-8 years posttransplant. The findings from these analyses reflect those of the body composition measures (Table 4-19). Similarly, HDL was inversely related to weight, while coronary risk ratio was positively related to weight.

A significant relationship was found with coronary risk ratio and weight $(r=0.33$; $p=0.03$ ). This implies that an increase in weight increases coronary risk ratio, which indicates that weight gain increases the coronary risk factors of developing CVD. This is consistent with previous findings showing how weight gain and CVD are linked (Akil \& Ahmad, 2011; Baum, 2001b; Kannel, D'Agostino, \& Cobb, 1996). Kannel et al. (1996) noted that being overweight worsens the CV risk profile. Baum (2001b) noted that $50 \%$ of the posttransplant population experienced weight gain. Weight gain is a major problem because of the interaction between obesity and immunosuppression medications, which increases the risk for CVD.

An inverse relationship exists with HDL and weight $(r=-0.46 ; p=0.001)$. This means that when weight is lowered, HDL increases, which is consistent with prior findings which show that weight reduction increases HDLand lowers LDL. Dattilo and Kris-Etherton (1992) studied the effects of weight reduction on blood lipids and lipoproteins in a meta-analysis. They found, from the 70 studies, that weight reduction lowered LDL and increased HDL. The relationship between HDL and BMI $(r=-0.34 ; p$ $=0.02$ ) is inverse. This suggests that a lower BMI is associated with an increased HDL.

\section{Research Question 5}

Research Question 5 asked to what degree changes in anthropometric measures from baseline to 5-8 years posttransplant are associated with serum glucose, lipids, and creatinine and elevated blood pressure at 5-8 years posttransplant. The only 
anthropometric measure found to be associated with the cardiac-related outcomes was BMI, which was associated with coronary risk ratio $(r=0.31 ; p=0.04)$. This finding was expected, given that obesity has long been recognized as a common risk factor for CVD (Dimeny, 2002; Ghoorah, Campbell, Kent, Maznyczka, \& Kunadian, 2016; Ladhani, Craig, Irving, Clayton, \& Wong, 2017; Lentine et al., 2008; Orazio et al., 2007). Moreover, obesity-related CVD is more common in kidney transplant recipients than in the general population (Marcén, 2006).

Although the change in BMI was only 3.11 from baseline $(M=29.03 ; S D=4.76)$ to 5-8 years posttransplant $(M=32.14 ; S D=9.61)$ in our cohort group, this represented an elevation to Class I obese (30-34.9). The degree of change, along with the probable trajectory of the gain, likely accounts for the significant finding. This finding is also consistent with previous findings (Beckmann, Ivanović, Drent, Ruppar, \& Geest, 2015; Cashion et al., 2007; Cupples et al., 2012; Nuttall, 2015; Stanfill et al., 2015) which found weight gain increasing by as much as $32 \mathrm{~kg}$ after 5-year post-kidney transplantation.

\section{Research Question 6}

Research Question 6 sought to determine the degree to which changes in body composition measures from baseline to 5-8 years posttransplant were associated with_serum glucose, lipids, creatinine, and elevated blood pressure. In contrast to anthropometric measures, which found only the change in BMI from time of transplant to 5-8 years later to be associated with coronary risk ratio, multiple measures of body mass were associated with creatinine, LDL, diastolic BP, triglycerides, and systolic BP (Tables 4-21 to 4-26).

All the relationships with creatinine (Table 4-21) were inverse, which is consistent with studies which have found that high body fat and high body mass can reduce excretion of creatinine resulting from the loss of muscle mass associated with aging and chronic illness (Heymsfield et al., 2005). In addition, Oh, Choi, Lee, \& Park (2017) have also reported that abdominal obesity reduces kidney function, which affects the kidney's ability to excrete waste products effectively. As Gerchman et al. (2009) stated, visceral adipose tissue found in the abdomen reduces the glomerular filtration rate, which reduces the clearance of creatinine from the kidney.

LDL was inversely related to three measures of lean tissue (total lean, gynoid lean, and visceral fat body lean) and positively related to total percent fat and gynoid percent fat. The relationships found with these compartments of lean tissue are consistent with previous findings that an increased lean body mass in the hips and thighs reduces LDL and increases the levels of HDL, thereby reducing the risk for CVD (Hioki et al., 2015; Hoenig, Cowin, Buckley, McHenery, \& Coulthard, 2011). Likewise our findings related to LDL, total percent fat, and gynoid percent fat are consistent with previous findings which reported that higher total percent is associated with LDL (Chiu, Williams \& Krauss [2017]) and findings by Min and Min (2014) that gynoid percent fat was 
positively correlated with LDL, indicating that an increase in gynoid percent fat accompanies an increase in LDL and, in return, an increased risk of CVD.

Diastolic BP was found to be related to both android fat $(r=0.30 ; p=0.05)$ and android mass $(r=0.33 p=0.03)$ as well as visceral fat and mass (Tables 4-22 to 4-26), indicating that increased weight in both android and visceral fat may contribute to increased diastolic BP. This finding is consistent with George et al. (2016), who studied the role of body fat and fat distribution in hypertension risk in urban Black South African women and found that trunk fat mass and arm fat mass accompanied increased diastolic BP levels. In the follow-up study to George et al. 5-8 years posttransplant, diastolic BP increased by $20.5 \%$. Yano et al. (2016) also studied regional fat distribution and found that an increase in VAT leads to an increase in diastolic pressure

An inverse relationship was found with systolic BP and android gynoid ratio $(r=$ $-0.40 ; p=0.01$ ), suggesting that a high $\mathrm{A} / \mathrm{G}$ ratio is associated with systolic $\mathrm{BP}$. This finding is surprising since one would expect that a high $\mathrm{A} / \mathrm{G}$ ratio would increase systolic $\mathrm{BP}$ rather than lower systolic BP.

\section{Strengths and Limitations}

This is the first longitudinal study of weight changes that occur in kidney transplant recipients to employ DXA scans to quantify measures of body composition. Although previous longitudinal studies have been conducted with similar samples, none have employed the same detailed measures of changes that occur in individual compartments of the body. In addition, this study expands upon the extant knowledge by examining the association and changes in body composition for their association with clinically important outcomes obtained 5 years plus posttransplant. The relationships found in this study provide important implications for practice and guidance for future research. Lastly, the BCAM model introduced to guide this study proved to be helpful in conceptualization, conduct, and analysis of the study findings. In addition to the BCAM model providing a valuable role in guiding future research, it may also aid HCPs in selecting the best methods to evaluate changes in $\mathrm{BC}$ and to more accurately tailor interventions considering individual biological and pathological conditions, thereby improving patient outcomes.

Despite the strength of the current study, some limitations existed. First, the small sample size $(N=45)$ may affect generalizability to a larger kidney transplant population. Second, study participants were not randomly selected but were selected from a parent study in the same transplant center of a large midsouth university-affiliated transplant institute, further threatening the generalizability of study findings. However, as an early exploratory study that is the first to use this longitudinal approach with detailed DXA scanning of body compartments, replication of this research should be undertaken. Third, a small sample size may increase the possibility of a Type II error (Burns \& Grove, 2009; Polit, 2010). A Type II error exists when the null hypothesis is false but is accepted as true from the statistical testing (Burn \& Grove, 2009; Polit, 2010). However, this is 
balanced to some degree by the large number of body composition measures, which could contribute to a Type I error. However, as an exploratory study, it is important to identify all potential contributing factors so they can be included in future, more fully powered and sophisticated explanatory modeling studies.

\section{Clinical and Practice Implications}

The results of the current study could serve as a work in process for future studies to target specific tissue measures for body fat and weight gain in the general and postkidney transplant population. Our work has found that commonly used indirect methods may be inappropriate for accurately accessing body fat and weight gain in the postkidney transplant population. In addition to the unique physiological conditions accompanying transplant that may affect distribution of weight, age, ethnicity/race, lifestyle, other clinical conditions influence the distribution of lean and fat mass across body compartments and thereby should also influence the choice of methods used to monitor body fat and weight. Clinical conditions may make certain indirect methods unsuitable for accurately assessing \%BF in individuals. For example, BIA measures assume total body water measurement is the same for all individuals; therefore, in obese or dehydrated individuals \%BF is overestimated (Dehghan \& Merchant, 2008; Heyward \& Wagner, 2004).

The BCAM is a science- and system-based model designed to guide practice decisions regarding selection of the best methods for measuring and monitoring weight. The BCAM illustrates a scientific-technological linkage between factors that influence weight gain and shows how the adverse changes in body composition affect the postkidney transplant population health outcomes. The conceptual framework of the BCAM can describe how body composition variation influences biological, lifestyle, healthrelated, and biometric factors in the presence of weight gain. The physiological changes affect body composition variation in the presence of abnormal lab levels of serum glucose, lipid, coronary artery ratio, and creatinine and long-standing elevated blood pressure, which could lead to mortality and morbidity. The findings from the current study show an association between weight gain and obesity-related cardiovascular disease and abnormal cardiac variables of the blood values.

\section{Recommendations for Future Research}

As a prospective correlational study, our results provide direction for future research on how the BCAM could support healthcare professionals in finding an appropriate indirect method to measure body fat and weight gain in the post-kidney transplant population. Additional research with larger representative samples of the postkidney transplant population is needed in order to generalize the study findings and to conduct more sophisticated multivariate analysis or predictive modeling. These additional studies will help provide more statistical insights into the measurement of components of 
body fat and weight gain and provide theoretical insight that will help to further develop the BCAM.

\section{Conclusion}

Postoperative weight gain is a common phenomenon in the kidney transplant population and is higher than in the general population. A weight gain of $10 \mathrm{~kg}$ in the first year and doubling to $32 \mathrm{~kg}$ in the second year is not unusual and often leads to obesityrelated complications. One of the obesity-related complications is the risk of developing cardiovascular disease that may lead to mortality in the kidney transplant population. Although various methods are used to measure and monitor weight gain over time, the literature lacks a consensus on which methods, specifically anthropometric or dualenergy x-ray absorptiometry, are most accurate in the assessment of weight gain in the presence of diseases in the kidney transplant population. Furthermore, limited studies have addressed the association between changes in body composition as measured by dual-energy x-ray absorptiometry or anthropometric indices and serum glucose, lipids, and blood pressure as predictors of cardiovascular disease after kidney transplantation.

The results from this study show several significant emerging risk factors of developing post-kidney transplant cardiovascular disease from excessive weight gain and obesity-related complications. Excessive weight gain and obesity-related complications are concerns in the kidney transplant population due to increased morbidity and mortality. Furthermore, the significant relationships found among the dual-energy x-ray absorptiometry and cardiac-related outcomes suggest that dual-energy x-ray absorptiometry provides a better assessment of body fat, weight gain, and potential cardiac risk factors than do the currently used anthropometric measures of weight and waist circumference. Thus, future research scientists and clinical practitioners should continue to examine the use of dual-energy x-ray absorptiometry for understanding the emerging coronary risk that accompanies weight gain and as a basis upon which more precise targeted interventions could be designed that would improve the health and life expectancy of kidney transplant patients. 


\section{LIST OF REFERENCES}

Akil, L., \& Ahmad, H. A. (2011). Relationships between obesity and cardiovascular diseases in four southern states and Colorado. $J$ Health Care Poor Underserved, 22(4 Suppl), 61-72. doi:10.1353/hpu.2011.0166

Aksoy, N. (2016). Weight gain after kidney transplant. Experimental and Clinical Transplantation, 3, 138-140. doi: 10.6002/ect.tondtdtd2016.P66

Alberti, K. G., Zimmet, P., \& Shaw, J. (2006). Metabolic syndrome--a new world-wide definition. A Consensus Statement from the International Diabetes Federation. Diabet Med, 23(5), 469-480. doi:10.1111/j.1464-5491.2006.01858.x

Allison, T. L. (2016). Immunosuppressive Therapy in Transplantation. Nurs Clin North Am, 51(1), 107-120. doi:10.1016/j.cnur.2015.10.008

Alshehri, A. M. (2010). Metabolic syndrome and cardiovascular risk. J Family Community Med, 17(2), 73-78. doi:10.4103/1319-1683.71987

Anjana, M., Sandeep, S., Deepa, R., Vimaleswaran, K. S., Farooq, S., \& Mohan, V. (2004). Visceral and central abdominal fat and anthropometry in relation to diabetes in Asian Indians. Diabetes Care, 27(12), 2948-2953.

Antillon, D., \& Towfighi, A. (2011). No time to 'weight': The link between obesity and stroke in women. Women's Health, 7(4), 453-463.

Armstrong, K. A., Campbell, S. B., Hawley, C. M., Johnson, D. W., \& Isbel, N. M. (2005). Impact of obesity on renal transplant outcomes. Nephrology, 10(4), 405413. doi:10.1111/j.1440-1797.2005.00406.x

Azancot, M. A., Ramos, N., Torres, I. B., Garcia-Carro, C., Romero, K., Espinel, E., . . Seron, D. (2015). Inflammation and Atherosclerosis Are Associated With Hypertension in Kidney Transplant Recipients. J Clin Hypertens (Greenwich), 17(12), 963-969. doi:10.1111/jch.12634

Ball, S. D., Altena, T. S., \& Swan, P. D. (2004). Comparison of anthropometry to DXA: a new prediction equation for men. Eur J Clin Nutr, 58(11), 1525-1531. doi:10.1038/sj.ejen.1602003

Bamoulid, J., Staeck, O., Halleck, F., Khadzhynov, D., Paliege, A., Brakemeier, S., . . . Budde, K. (2016). Immunosuppression and Results in Renal Transplantation. European Urology Supplements, 15(9), 415-429. doi:10.1016/j.eursup.2016.04.011

Banerji, M. A., Faridi, N., Atluri, R., Chaiken, R. L., \& Lebovitz, H. E. (1999). Body composition, visceral fat, leptin, and insulin resistance in Asian Indian men. $J$ Clin Endocrinol Metab, 84(1), 137-144. doi:10.1210/jcem.84.1.5371

Baum, C. L. (2001a). Weight Gain and Cardiovascular Risk After Organ Transplantation. JPEN Journal of Parenteral \& Enteral Nutrition, 25(3), 114.

Baum, C. L. (2001b). Weight gain and cardiovascular risk after organ transplantation. JPEN J Parenter Enteral Nutr, 25(3), 114-119. doi:10.1177/0148607101025003114

Baum, C. L., Thielke, K., Westin, E., Kogan, E., Cicalese, L., \& Benedetti, E. (2002). Predictors of weight gain and cardiovascular risk in a cohort of racially diverse kidney transplant recipients. Nutrition, 18(2), 139-146. 
Baumgartner, R. N., Heymsfield, S. B., Lichtman, S., Wang, J., \& Pierson, R. N., Jr. (1991). Body composition in elderly people: effect of criterion estimates on predictive equations. Am J Clin Nutr, 53(6), 1345-1353.

Beckmann, S., Ivanović, N., Drent, G., Ruppar, T., \& De Geest, S. (2015). Weight gain, overweight and obesity in solid organ transplantation - a study protocol for a systematic literature review. (article), Retrieved from

http://ezproxy.uthsc.edu/login?url=http://search.ebscohost.com/login.aspx?direct= true $\& \mathrm{db}=\mathrm{ddu} \& \mathrm{AN}=5951419176954 \mathrm{CC}$ \& $\&$ site $=$ eds-live Available from EBSCOhost ddu database.

Beckmann, S., Ivanović, N., Drent, G., Ruppar, T., \& Geest, S. D. (2015). Weight gain, overweight and obesity in solid organ transplantation - a study protocol for a systematic literature review. Systematic Reviews, 4(1), 2. doi:10.1186/2046-40534-2

Bellizzi, V., Cupisti, A., Capitanini, A., Calella, P., \& D'Alessandro, C. (2014). Physical Activity and Renal Transplantation. Kidney and Blood Pressure Research, 39(23), 212-219. doi:10.1159/000355799

Bergman, R. N., Kim, S. P., Catalano, K. J., Hsu, I. R., Chiu, J. D., Kabir, M., . . Ader, M. (2006). Why visceral fat is bad: mechanisms of the metabolic syndrome. Obesity (Silver Spring), 14 Suppl 1, 16S-19S. doi:10.1038/oby.2006.277

Bloodworth, R. F., Ward, K. D., Relyea, G. E., \& Cashion, A. K. (2014). Food availability as a determinant of weight gain among renal transplant recipients. Res Nurs Health, 37(3), 253-259. doi:10.1002/nur.21599

Burns, N. \& Grove, S. K.. (2009). The practice of nursing research: Appraisal, synthesis, and generation of evidence. ( $6^{\text {th }}$ ed.). St. Louis, MO: Saunders.

Calò, L. A., Ravarotto, V., Simioni, F., Naso, E., Marchini, F., Bonfante, L., . . Rigotti, P. (2017). Pathophysiology of Post Transplant Hypertension in Kidney Transplant: Focus on Calcineurin Inhibitors Induced Oxidative Stress and Renal Sodium Retention and Implications with RhoA/Rho Kinase Pathway. Kidney and Blood Pressure Research, 42(4), 676-685.

Carpenter, C. L., Yan, E., Chen, S., Hong, K., Arechiga, A., Kim, W. S., ...Heber, D. (2013). Body fat and body-mass index among a multiethnic sample of college-age men and women. J Obes, 2013, 80-654. doi:10.1155/2013/790654.

Cashion, A. K., Hathaway, D. K., Stanfill, A., Thomas, F., Ziebarth, J. D., Cui, Y., . . Eason, J. (2014). Pre-transplant Predictors of One Year Weight Gain After Kidney Transplantation. Clinical Transplantation, 28(11), 1271-1278. doi:10.1111/ctr.12456

Cashion, A. K., Sanchez, Z. V., Cowan, P. A., Hathaway, D. K., Lo Costello, A., \& Gaber, A. O. (2007). Changes in weight during the first year after kidney transplantation. Prog Transplant, 17(1), 40-47.

Castelli, W. P., Doyle, J. T., Gordon, T., Hames, C. G., Hjortland, M. C., Hulley, S. B., . . . Zukel, W. J. (1977). HDL cholesterol and other lipids in coronary heart disease. The cooperative lipoprotein phenotyping study. Circulation, 55(5), 767-772.

Castelli, W. P., Garrison, R. J., Wilson, P. W., Abbott, R. D., Kalousdian, S., \& Kannel, W. B. (1986). Incidence of coronary heart disease and lipoprotein cholesterol levels. The Framingham Study. Jama, 256(20), 2835-2838. 
Catenacci, V. A., Hill, J. O., \& Wyatt, H. R. (2009). The obesity epidemic. Clin Chest Med, 30(3), 415-444, vii. doi:10.1016/j.ccm.2009.05.001

Centers for Disease Control and Prevention. (2014). The CDC guide to increased physical activity in the community. Retrieved from http://www.cdc.gov/obesity/downloadsPA_2011_WEB.pdf

Centers for Disease Control and Prevention. (2014). The CDC guide to strategies to increase physical activity in the community. Retrieved from http://www.cdc.gov/obesity/downloads/PA_2011_WEB.pdf

Centers for Disease Control and Prevention (2014). National Diabetes Statistics Report, 2014 - CDC. Retrieved from https://www.cdc.gov/diabetes/pdfs/data/2014-reportestimates of diabetes and its burden in the united states.pdf

Centers for Disease Control and Prevention (2014). National Diabetes Statistics Report, 2017 - CDC. Retrieved from https://www.cdc.gov/diabetes/pdfs/data/statistics/national-diabetes-statisticsreport.pdf

Chan, G., Garneau, P., \& Hajjar, R. (2015). The impact and treatment of obesity in kidney transplant candidates and recipients. Canadian Journal of Kidney Health and Disease, 2, 26. doi:10.1186/s40697-015-0059-4

Chiu, S., Williams, P. T., \& Krauss, R. M. (2017). Effects of a very high saturated fat diet on LDL particles in adults with atherogenic dyslipidemia: A randomized controlled trial. PLOS ONE, 12(2), e0170664. doi:10.1371/journal.pone.0170664

Clunk, J. M., Lin, C. Y., \& Curtis, J. J. (2001). Variables affecting weight gain in renal transplant recipients. Am J Kidney Dis, 38(2), 349-353. doi:10.1053/ajkd.2001.26100

Cofan, F., Vela, E., \& Cleries, M. (2005). Obesity in renal transplantation: analysis of 2691 patients. Transplant Proc, 37(9), 3695-3697. doi:10.1016/j.transproceed.2005.10.069

Cooper, B. A., Aslani, A., Ryan, M., Zhu, F. Y, Ibels, L.S., Allen, B. J., \& Pollock, C. A. (2000). Comparing different methods of assessing body composition in end-stage renal failure. Kidney International, 58(1), 408-16. doi: 10.1046/j.15231755.2000.00180.x

Cordeiro, A. C., Qureshi, A. R., Lindholm, B., Amparo, F. C., Tito-Paladino-Filho, A., Perini, M., . . Carrero, J. J. (2013). Visceral fat and coronary artery calcification in patients with chronic kidney disease. Nephrology Dialysis Transplantation, 28(suppl_4), iv152-iv159. doi:10.1093/ndt/gft250

Cosio, F. G., Pelletier, R. P., Pesavento, T. E., Henry, M. L., Ferguson, R. M., Mitchell, L., \& Lemeshow, S. (2001). Elevated blood pressure predicts the risk of acute rejection in renal allograft recipients. Kidney International, 59(3), 1158-1164. doi:https://doi.org/10.1046/j.1523-1755.2001.0590031158.x

Cupples, C. K., Cashion, A. K., Cowan, P. A., Tutor, R. S., Wicks, M. N., Williams, R., \& Eason, J. D. (2012). Characterizing dietary intake and physical activity affecting weight gain in kidney transplant recipients. Prog Transplant, 22(1), 6270.

Daniels, S. R. (2009). The Use of BMI in the Clinical Setting. Pediatrics, 124(Supplement 1), S35. 
Dattilo, A. M., \& Kris-Etherton, P. M. (1992). Effects of weight reduction on blood lipids and lipoproteins: a meta-analysis. Am J Clin Nutr, 56(2), 320-328. doi:10.1093/ajen/56.2.320

de Oliveira, C. M. C., Moura, Á. E. F., Gonçalves, L., Pinheiro, L. S. F., Pinheiro, F. M. L., \& Esmeraldo, R. M. (2014). Post-Transplantation Weight Gain: Prevalence and the Impact of Steroid-Free Therapy. Transplant Proc, 46(6), 1735-1740. doi:https://doi.org/10.1016/j.transproceed.2014.05.027

Dehghan, M., \& Merchant, A. T. (2008). Is bioelectrical impedance accurate for use in large epidemiological studies? Nutr J, 7, 26. doi:10.1186/1475-2891-7-26

Despres, J. P. (2012). Body fat distribution and risk of cardiovascular disease: an update. Circulation, 126(10), 1301-1313. doi:10.1161/circulationaha.111.067264

Despres, J. P., Moorjani, S., Lupien, P. J., Tremblay, A., Nadeau, A., \& Bouchard, C. (1990). Regional distribution of body fat, plasma lipoproteins, and cardiovascular disease. Arteriosclerosis, 10(4), 497-511.

DiCecco, S. R., \& Francisco-Ziller, N. (2014). Obesity and organ transplantation: successes, failures, and opportunities. Nutr Clin Pract, 29(2), 171-191. doi: $10.1177 / 0884533613518585$

Dimeny, E. M. (2002). Cardiovascular disease after renal transplantation. Kidney Int Suppl(80), 78-84. doi:10.1046/j.1523-1755.61.s80.14.x

Donato, G. B., Fuchs, S. C., Oppermann, K., Bastos, C., \& Spritzer, P. M. (2006). Association between menopause status and central adiposity measured at different cutoffs of waist circumference and waist-to-hip ratio. Menopause, 13(2), 280-285. doi:10.1097/01.gme.0000177907.32634.ae

Dontje, M. L., Greef, M. H. G., Krijnen, W. P., Corpeleijn, E., Kok, T., Bakker, S. J. L., . . . Schans, C. P. (2014). Longitudinal measurement of physical activity following kidney transplantation. Clinical Transplantation, 28(4), 394-402. doi: $10.1111 /$ ctr. 12325

Dordevic, A. L., Bonham, M., Ghasem-Zadeh, A., Evans, A., Barber, E., Day, K., . . . Truby, H. (2018). Reliability of Compartmental Body Composition Measures in Weight-Stable Adults Using GE iDXA: Implications for Research and Practice. Nutrients, 10(10). doi:10.3390/nu10101484

Duren, D. L., Sherwood, R. J., Czerwinski, S. A., Lee, M., Choh, A. C., Siervogel, R. M., \& Cameron Chumlea, W. (2008). Body Composition Methods: Comparisons and Interpretation. Journal of diabetes science and technology (Online), 2(6), 11391146.

Eckel, R. H., Kahn, S. E., Ferrannini, E., Goldfine, A. B., Nathan, D. M., Schwartz, M. W., ... Smith, S. R. (2011). Obesity and type 2 diabetes: what can be unified and what needs to be individualized? J Clin Endocrinol Metab, 96(6), 1654-1663. doi:10.1210/jc.2011-0585

Elli, A., Traversi, L., \& Ponticelli, C. (2000). Cardiovascular Risk Factors in Renal Transplant Recipients. The International Journal of Artificial Organs, 23(11), 730-735. doi:10.1177/039139880002301102

Elster, E. A., Leeser, D. B., Morrissette, C., Pepek, J. M., Quiko, A., Hale, D. A., . . Mannon, R. B. (2008). Obesity following kidney transplantation and steroid avoidance immunosuppression. Clin Transplant, 22(3), 354-359. doi:10.1111/j.1399-0012.2008.00792.x 
Fernandes, J. F. R., Leal, P. M., Rioja, S., Bregman, R., Sanjuliani, A. F., Barreto Silva, M. I., \& Torres, M. R. S. G. (2013). Adiposity and cardiovascular disease risk factors in renal transplant recipients: Are there differences between sexes? Nutrition, 29(10), 1231-1236. doi:10.1016/j.nut.2013.03.019

Flegal, K. M., Carroll, M. D., Kuczmarski, R. J., \& Johnson, C. L. (1998). Overweight and obesity in the United States: prevalence and trends, 1960-1994. Int J Obes Relat Metab Disord, 22(1), 39-47.

Fu, X., Song, A., Zhou, Y., Ma, X., Jiao, J., Yang, M., \& Zhu, S. (2014). Association of regional body fat with metabolic risks in Chinese women. Public Health Nutr, 17(10), 2316-2324. doi:10.1017/S1368980013002668

Gallagher, D., Heymsfield, S. B., Heo, M., Jebb, S. A., Murgatroyd, P. R., \& Sakamoto, Y. (2000a). Healthy percentage body fat ranges: an approach for developing guidelines based on body mass index. Am J Clin Nutr, 72(3), 694-701. doi:10.1093/ajen/72.3.694

Gallagher, D., Heymsfield, S. B., Heo, M., Jebb, S. A., Murgatroyd, P. R., \& Sakamoto, Y. (2000b). Healthy percentage body fat ranges: an approach for developing guidelines based on body mass index. American Journal of Clinical Nutrition, 72(3), 694-701.

Gao, S. W., Oliver, D. K., Das, N., Hurst, F. P., Lentine, K. L., Agodoa, L. Y., . . . Abbott, K. C. (2008). Assessment of racial disparities in chronic kidney disease stage 3 and 4 care in the department of defense health system. Clin J Am Soc Nephrol, 3(2), 442-449. doi:10.2215/CJN.03940907

George, C., Goedecke, J. H., Crowther, N. J., Jaff, N. G., Kengne, A. P., Norris, S. A., \& Micklesfield, L. K. (2016). The Role of Body Fat and Fat Distribution in Hypertension Risk in Urban Black South African Women. PLOS ONE, 11(5), e0154894. doi:10.1371/journal.pone.0154894

Gerchman, F., Tong, J., Utzschneider, K. M., Zraika, S., Udayasankar, J., McNeely, M. J., . . . De Boer, I. H. (2009). Body mass index is associated with increased creatinine clearance by a mechanism independent of body fat distribution. The Journal of Clinical Endocrinology \& Metabolism, 94(10), 3781-3788.

Ghoorah, K., Campbell, P., Kent, A., Maznyczka, A., \& Kunadian, V. (2016). Obesity and cardiovascular outcomes: a review. Eur Heart J Acute Cardiovasc Care, 5(1), 77-85. doi:10.1177/2048872614523349

Gill, T. P. (2001). Cardiovascular risk in the Asia-Pacific region from a nutrition and metabolic point of view: abdominal obesity. Asia Pac J Clin Nutr, 10(2), 85-89.

Gonzalez, M. C., Orlandi, S. P., Santos, L. P., \& Barros, A. J. D. (2018). Body composition using bioelectrical impedance: Development and validation of a predictive equation for fat-free mass in a middle-income country. Clin Nutr. doi:10.1016/j.clnu.2018.09.012

Gore, J. L., Pham, P. T., Danovitch, G. M., Wilkinson, A. H., Rosenthal, J. T., Lipshutz, G. S., \& Singer, J. S. (2006). Obesity and outcome following renal transplantation. Am J Transplant, 6(2), 357-363. doi:10.1111/j.16006143.2005.01198.x

Guerra, R. S., Amaral, T. F., Marques, E., Mota, J., \& Restivo, M. T. (2010). Accuracy of Siri and Brozek equations in the percent body fat estimation in older adults. Journal of Nutrition, Health \& Aging, 14(9), 744. 
Han, S. S., Kim, K. W., Kim, K.-I., Na, K. Y., Chae, D.-W., Kim, S., \& Chin, H. J. (2010). Lean Mass Index: A Better Predictor of Mortality than Body Mass Index in Elderly Asians. Journal of the American Geriatrics Society, 58(2), 312-317. doi:10.1111/j.1532-5415.2009.02672.x

Hewing, B., Moore, K. J., \& Fisher, E. A. (2012). HDL and cardiovascular risk: time to call the plumber? Circ Res, 111(9), 1117-1120.

doi:10.1161/circresaha.112.280958

Heymsfield, S. B., Lohman, T. G., Wang, Z., \& Going, S. B. (2005). Human Body Composition

Heymsfield, S. B., Peterson, C. M., Thomas, D. M., Heo, M., \& Schuna, J. M., Jr. (2016). Why are there race/ethnic differences in adult body mass index-adiposity relationships? A quantitative critical review. Obes Rev, 17(3), 262-275. doi:10.1111/obr.12358

Heymsfield, S. B., Wang, Z., Baumgartner, R. N., \& Ross, R. (1997). Human body composition: advances in models and methods. Annu Rev Nutr, 17, 527-558. doi:10.1146/annurev.nutr.17.1.527

Heyward, V. H., \& Wagner, D. R. (2004). Applied Body Composition Assessment (2nd ed. ed.). Champaign, IL: Human Kinetics.

Hioki, H., Miura, T., Motoki, H., Kobayashi, H., Kobayashi, M., Nakajima, H., . . I Ikeda, U. (2015). Lean body mass index prognostic value for cardiovascular events in patients with coronary artery disease. Heart Asia, 7(2), 12-18. doi:10.1136/heartasia-2015-010644

Hoenig, M. R., Cowin, G., Buckley, R., McHenery, C., \& Coulthard, A. (2011). Low density lipoprotein cholesterol is inversely correlated with abdominal visceral fat area: a magnetic resonance imaging study. Lipids in Health and Disease, 10, 1212. doi:10.1186/1476-511X-10-12

Hoorn, E. J., Walsh, S. B., McCormick, J. A., Zietse, R., Unwin, R. J., \& Ellison, D. H. (2012). Pathogenesis of calcineurin inhibitor-induced hypertension. Journal of nephrology, 25(3), 269.

Huang, B. T., Peng, Y., Liu, W., Zhang, C., Huang, F. Y., Wang, P. J., . . . Chen, M. (2015). Lean mass index, body fat and survival in Chinese patients with coronary artery disease. QJM: An International Journal of Medicine, 108(8), 641-647. doi:10.1093/qjmed/hcv013

Jensen, M. D. (2008). Role of body fat distribution and the metabolic complications of obesity. J Clin Endocrinol Metab, 93(11 Suppl 1), S57-63. doi:10.1210/jc.20081585

Jezior, D., Krajewska, M., Madziarska, K., Regulska-Ilow, B., Ilow, R., Janczak, D., . . . Klinger, M. (2007). Weight reduction in renal transplant recipients program: the first successes. Transplant Proc, 39(9), 2769-2771.

doi:10.1016/j.transproceed.2007.08.055

Johnson, C. P., Gallagher-Lepak, S., Zhu, Y. R., Porth, C., Kelber, S., Roza, A. M., \& Adams, M. B. (1993). Factors influencing weight gain after renal transplantation. Transplantation, 56(4), 822-827.

Josse, A. R., Azizian, H., French, S. B., Kramer, J. K., \& Phillips, S. M. (2011). Body fat content determination in premenopausal, overweight, and obese young women 
using DXA and FT-NIR. Obesity (Silver Spring), 19(7), 1497-1502.

doi:10.1038/oby.2011.39

Journal of the Americal College of Cardiology, May 2018, 71(19) e127-e248; doi:10.1016/j.jacc.2017.11.006

Jun, M., Lv, J., Perkovic, V., \& Jardine, M. J. (2011). Managing Cardiovascular Risk in People with Chronic Kidney Disease: A Review of the Evidence from Randomized Controlled Trials. Therapeutic Advances in Chronic Disease, 2(4), 265-278. doi:10.1177/2040622311401775

Jura, M., \& Kozak, L. P. (2016). Obesity and related consequences to aging. Age, 38(1), 23. http://doi.org/10.1007/s11357-016-9884-3.

Kalluri, H. V., \& Hardinger, K. L. (2012). Current state of renal transplant immunosuppression: Present and future. World Journal of Transplantation, 2(4), 51-68. doi:10.5500/wjt.v2.i4.51

Kang, S. H., Cho, K. H., Park, J. W., Yoon, K. W., \& Do, J. Y. (2014). Body composition measurements using bioimpedance analysis in peritoneal dialysis patients are affected by the presence of dialysate. Nephrology, 19(11), 727-731. doi:10.1111/nep. 12322

Kannel, W. B., D'Agostino, R. B., \& Cobb, J. L. (1996). Effect of weight on cardiovascular disease. Am J Clin Nutr, 63(3 Suppl), 419s-422s. doi:10.1093/ajen/87.6.1602

Kathryn, N. P. S., Shelley, R. M., Julia, A. W., \& Connie, W. B. (2016). Challenges in the Management of Geriatric Obesity in High Risk Populations. Nutrients, Vol 8, IsS 5, $p 262$ (2016)(5), 262. doi:10.3390/nu8050262

Kim, Y., Kim, J.-R., Choi, H., Hwang, J.-w., Jang, H. R., Lee, J. E., . . Huh, W. (2013). Patients with Persistent New-Onset Diabetes after Transplantation Have Greater Weight Gain after Kidney Transplantation. J Korean Med Sci, 28(10), 1431-1434.

Kissebah, A. H., Vydelingum, N., Murray, R., Evans, D. J., Hartz, A. J., Kalkhoff, R. K., \& Adams, P. W. (1982). Relation of body fat distribution to metabolic complications of obesity. J Clin Endocrinol Metab, 54(2), 254-260. doi: $10.1210 /$ jcem-54-2-254

Kouda, K., Nakamura, H., Fujita, Y., Ohara, K., \& Iki, M. (2012). Increased ratio of trunk to appendicular fat and increased blood pressure: study of a general population of Hamamatsu children. Circ J, 76(12), 2848-2854.

Kwakernaak, A. J., Toering, T. J., \& Navis, G. (2013). Body mass index and body fat distribution as renal risk factors: a focus on the role of renal haemodynamics. Nephrol Dial Transplant, 28 Suppl 4, iv42-49. doi:10.1093/ndt/gft331

Kwan, J. M., Hajjiri, Z., Metwally, A., Finn, P. W., \& Perkins, D. L. (2016). Effect of the Obesity Epidemic on Kidney Transplantation: Obesity Is Independent of Diabetes as a Risk Factor for Adverse Renal Transplant Outcomes. PLOS ONE, 11(11), e0165712. doi:10.1371/journal.pone.0165712

Ladhani, M., Craig, J. C., Irving, M., Clayton, P. A., \& Wong, G. (2017). Obesity and the risk of cardiovascular and all-cause mortality in chronic kidney disease: a systematic review and meta-analysis. Nephrol Dial Transplant, 32(3), 439-449. doi:10.1093/ndt/gfw075 
Lafranca, J. A., IJermans, J. N., Betjes, M. G., \& Dor, F. J. (2015a). Body mass index and outcome in renal transplant recipients: a systematic review and meta-analysis. BMC Medicine, 13(1), 111. doi:10.1186/s12916-015-0340-5

Lafranca, J. A., Ijermans, J. N. M., Betjes, M. G. H., \& Dor, F. J. M. F. (2015b). Body mass index and outcome in renal transplant recipients: a systematic review and meta-analysis. BMC Medicine, 13(1), 111. doi:10.1186/s12916-015-0340-5

Latt, E., Maestu, J., \& Jurimae, J. (2018). Longitudinal associations of android and gynoid fat mass on cardiovascular disease risk factors in normal weight and overweight boys during puberty. Am J Hum Biol, e23171. doi:10.1002/ajhb.23171

Lavie, C. J., De Schutter, A., Patel, D., Artham, S. M., \& Milani, R. V. (2011). Body Composition and Coronary Heart Disease Mortality-An Obesity or a Lean Paradox? Mayo Clinic Proceedings, 86(9), 857-864. doi:10.4065/mcp.2011.0092

Lee, S. Y., \& Gallagher, D. (2008). Assessment methods in human body composition. Curr Opin Clin Nutr Metab Care, 11(5), 566-572. doi:10.1097/MCO.0b013e32830b5f23

Lentine, K. L., Hurst, F. P., Jindal, R. M., Villines, T. C., Kunz, J. S., Yuan, C. M., . . Abbott, K. C. (2010). Cardiovascular Risk Assessment Among Potential Kidney Transplant Candidates: Approaches and Controversies. American Journal of Kidney Diseases, 55(1), 152-167. doi:https://doi.org/10.1053/j.ajkd.2009.06.032

Lentine, K. L., Rocca-Rey, L. A., Bacchi, G., Wasi, N., Schmitz, L., Salvalaggio, P. R., . . Brennan, D. C. (2008). Obesity and cardiac risk after kidney transplantation: experience at one center and comprehensive literature review. Transplantation, 86(2), 303-312. doi:10.1097/TP.0b013e31817ef0f9

MacLean, P. S., Higgins, J. A., Giles, E. D., Sherk, V. D., \& Jackman, M. R. (2015). The role for adipose tissue in weight regain after weight loss. Obes Rev, 16 Suppl 1, 45-54. doi:10.1111/obr.12255

Mafutha, G. N., \& Wright, S. C. (2013). Compliance or non-compliance of hypertensive adults to hypertension management at three primary healthcare day clinics in Tshwane. Curationis, 36(1), E1-6.

Maraghi, E., Rahimi Foroushani, A., Younespour, S., Rostami, Z., Einollahi, B., Eshraghian, M. R., . . . Mohammad, K. (2016). Longitudinal Assessment of Serum Creatinine Levels on Graft Survival After Renal Transplantation: Joint Modeling Approach. Nephro-urology Monthly, 8(4), e37666. doi:10.5812/numonthly.37666

Marcén, R. (2006). Cardiovascular risk factors in renal transplantation-current controversies. Nephrology Dialysis Transplantation, 21(suppl_3), iii3-iii8. doi:10.1093/ndt/gfl298

McPartland, K. J., \& Pomposelli, J. J. (2007). Update on immunosuppressive drugs used in solid-organ transplantation and their nutrition implications. Nutr Clin Pract, 22(5), 467-473. doi:10.1177/0115426507022005467

Min, K.-B., \& Min, J.-Y. (2014). Android and gynoid fat percentages and serum lipid levels in United States adults. Clinical Endocrinology, 82(3), 377-387. doi:10.1111/cen.12505

Mohammadifard, N., Nazem, M., Sarrafzadegan, N., Nouri, F., Sajjadi, F., Maghroun, M., \& Alikhasi, H. (2013). Body mass index, waist-circumference and 
cardiovascular disease risk factors in Iranian adults: Isfahan healthy heart program. J Health Popul Nutr, 31(3), 388-397.

Moore, L. W., \& Gaber, A. O. (1996). Patterns of early weight change after renal transplantation. Journal of Renal Nutrition, 6(1), 21-25. doi:10.1016/S10512276(96)90104-1

Murray, S. (2006). Is waist-to-hip ratio a better marker of cardiovascular risk than body mass index? CMAJ : Canadian Medical Association Journal, 174(3), 308-308. doi:10.1503/cmaj.051561

Naumnik, B., \& Mysliwiec, M. (2010). Renal consequences of obesity. Med Sci Monit, 16(8), Ra163-170.

Neale, J., \& Smith, A. C. (2015). Cardiovascular risk factors following renal transplant. World Journal of Transplantation, 5(4), 183-195. doi:10.5500/wjt.v5.i4.183

Ng, B. K., Liu, Y. E., Wang, W., Kelly, T. L., Wilson, K. E., Schoeller, D. A., . . . Shepherd, J. A. (2018). Validation of rapid 4-component body composition assessment with the use of dual-energy X-ray absorptiometry and bioelectrical impedance analysis. Am J Clin Nutr, 108(4), 708-715. doi:10.1093/ajcn/nqy158

Nuttall, F. Q. (2015). Body Mass Index: Obesity, BMI, and Health: A Critical Review. Nutr Today, 50(3), 117-128. doi:10.1097/nt.0000000000000092

O'Brien, T., \& Hathaway, D. (2016). An Integrative Literature Review of Physical Activity Recommendations for Adult Renal Transplant Recipients. Prog Transplant, 26(4), 381-385. doi:10.1177/1526924816664079

Ogden, C. L., Carroll, M. D., Kit, B. K., \& Flegal, K. M. (2013). Prevalence of obesity among adults: United States, 2011-2012. NCHS Data Brief(131), 1-8.

Ogden, C. L., Carroll, M. D., Kit, B. K., \& Flegal, K. M. (2014). Prevalence of childhood and adult obesity in the United States, 2011-2012. Jama, 311(8), 806-814. doi:10.1001/jama.2014.732

Oh, I. H., Choi, J. W., Lee, C. H., \& Park, J.-S. (2017). Estimating Negative Effect of Abdominal Obesity on Mildly Decreased Kidney Function Using a Novel Index of Body-Fat Distribution. J Korean Med Sci, 32(4), 613-620.

Ojo, A. O., Hanson, J. A., Wolfe, R. A., Leichtman, A. B., Agodoa, L. Y., \& Port, F. K. (2000). Long-term survival in renal transplant recipients with graft function. Kidney Int, 57(1), 307-313. doi:10.1046/j.1523-1755.2000.00816.x

Olarte, I. G., \& Hawasli, A. (2009). Kidney transplant complications and obesity. Am J Surg, 197(3), 424-426. doi:10.1016/j.amjsurg.2008.11.021

Olyaei, A. J., deMattos, A. M., \& Bennett, W. M. (1999). A Practical Guide to the Management of Hypertension in Renal Transplant Recipients. Drugs, 58(6), 1011-1027. doi:10.2165/00003495-199958060-00005

Orazio, L., Armstrong, K., Banks, M., Johnson, D., Isbel, N., \& Hickman, I. (2007). Central obesity is common in renal transplant recipients and is associated with increased prevalence of cardiovascular risk factors. Nutrition \& Dietetics, 64(3), 200-206. doi:10.1111/j.1747-0080.2007.00151.x

Pantik, C., Cho, Y. E., Hathaway, D., Tolley, E., \& Cashion, A. (2017). Characterization of Body Composition and Fat Mass Distribution 1 Year After Kidney Transplantation. Prog Transplant, 27(1), 10-15. doi:10.1177/1526924816681007 
Paripovic, D., Kostic, M., Spasojevic, B., Kruscic, D., \& Peco-Antic, A. (2010a). Masked hypertension and hidden uncontrolled hypertension after renal transplantation. Pediatr Nephrol, 25(9), 1719-1724. doi:10.1007/s00467-010-1552-8

Paripovic, D., Kostic, M., Spasojevic, B., Kruscic, D., \& Peco-Antic, A. (2010b). Masked hypertension and hidden uncontrolled hypertension after renal transplantation. Pediatric Nephrology, 25(9), 1719-1724. doi:10.1007/s00467-010-1552-8

Peev, V., Reiser, J., \& Alachkar, N. (2014). Diabetes Mellitus in the Transplanted Kidney. Frontiers in Endocrinology, 5, 141.

Pham, P. T., Pham, P. M., Pham, S. V., Pham, P. A., \& Pham, P. C. (2011). New onset diabetes after transplantation (NODAT): an overview. Diabetes Metab Syndr Obes, 4, 175-186. doi:10.2147/dmso.S19027

Pi-Sunyer, X. (2018). Changes in body composition and metabolic disease risk. Eur J Clin Nutr. doi:10.1038/s41430-018-0320-x

Polit, D. F. (2010). Statistics and data analysis for nursing research (2 $\left.{ }^{\text {nd }} \mathrm{ed}.\right)$. New York, NY: Pearson/Prentice Hall.

Puri, A., Singh, V., Pandey, S., Singh, S. R. K., Singh, C., \& Srivastav, R. (2014). Measurement of body fat percentage and visceral fat rating using bioelectrical impedance analysis in coronary artery disease patients and its associations with aggressive lipid lowering treatment. Journal of Indian College of Cardiology, 4(1), 1-7. doi:https://doi.org/10.1016/j.jicc.2013.11.001

Purnell, J. Q., Kahn, S. E., Albers, J. J., Nevin, D. N., Brunzell, J. D., \& Schwartz, R. S. (2000). Effect of Weight Loss with Reduction of Intra-Abdominal Fat on Lipid Metabolism in Older Men*. The Journal of Clinical Endocrinology \& Metabolism, 85(3), 977-982. doi:10.1210/jcem.85.3.6402

Rao, N. N., \& Coates, P. T. (2018). Cardiovascular Disease After Kidney Transplant. Semin Nephrol, 38(3), 291-297. doi:10.1016/j.semnephrol.2018.02.008

Richmond, T. K., Thurston, I., Sonneville, K., Milliren, C. E., Walls, C. E. \& Austin, S. B. (2015). Racial/ethnic differences in accuracy of body mass index reporting in a diverse cohort of young adults. International Journal of Obseidty, 39(3), 546-548. https://doi.10.1038/ijo.2014.147.

Robic, T, Benedik, E., Mis, N. F., Bratanic, B., \& Petra, I. R. (2018). Challenges in determining body fat in pregnant women. Annals of Nutrition \& Metabolism, 63, 341-349. doi:10.1159/000358339

Ross, R., \& Janiszewski, P. M. (2008). Is weight loss the optimal target for obesityrelated cardiovascular disease risk reduction? Can J Cardiol, 24 Suppl D, 25d$31 \mathrm{~d}$.

Ryan, K. J., Casas, J. M., Mash, L. E., McLellan, S. L., Lloyd, L. E., Stinear, J. W., . . . Collins, M. G. (2014). The effect of intensive nutrition interventions on weight gain after kidney transplantation: protocol of a randomised controlled trial. $B M C$ Nephrol, 15, 148. doi:10.1186/1471-2369-15-148

Saemann, M. D., \& Sunder-Plassmann, G. (2008). Maintenance immunosuppressive therapy in adult renal transplantation: a single center analysis. Transpl Immunol, 20(1-2), 14-20. doi:10.1016/j.trim.2008.08.012

Sanchez, Z. V., Cashion, A. K., Cowan, P. A., Jacob, S. R., Wicks, M. N., \& VelasquezMieyer, P. (2007). Perceived barriers and facilitators to physical activity in kidney transplant recipients. Prog Transplant, 17(4), 324-331. 
Scherrer, U., Nussberger, J., Torriani, S., Waeber, B., Darioli, R., Hofstetter, J. R., \& Brunner, H. R. (1991). Effect of weight reduction in moderately overweight patients on recorded ambulatory blood pressure and free cytosolic platelet calcium. Circulation, 83(2), 552-558.

Schoeller, D. A., Tylavsky, F. A., Baer, D. J., Chumlea, W. C., Earthman, C. P., Fuerst, T., . . Borrud, L. G. (2005). QDR 4500A dual-energy X-ray absorptiometer underestimates fat mass in comparison with criterion methods in adults. American Journal of Clinical Nutrition, 81(5), 1018.

Shah, N. R., \& Braverman, E. R. (2012). Measuring adiposity in patients: the utility of body mass index (BMI), percent body fat, and leptin. PLOS ONE, 7(4), e33308. doi:10.1371/journal.pone.0033308

Sharp, D. S., Andrew, M. E., Burchfiel, C. M., Violanti, J. M., \& Wactawski-Wende, J. Body mass index versus dual energy $\mathrm{x}$-ray absorptiometry-derived indexes: Predictors of cardiovascular and diabetic disease risk factors. American Journal of Human Biology, 24(4), 400-405. doi: 10.1002/ajhb.22221

Shaw, K. A., Srikanth, V. K., Fryer, J. L., Blizzard, L., Dwyer, T., \& Venn, A. J. (2007). Dual energy X-ray absorptiometry body composition and aging in a populationbased older cohort. International Journal of Obesity, 31, 279-284. doi: $10.1038 /$ sj.ijo. 0803417

Sheskin, D. J. (2011). Handbook of parametric and nonparametric statistical procedures ( $5^{\text {th }}$ ed.). Boca Raton, FL: Chapman \& Hall/CRC Press.

Simmons, K. P. (2001). Body measurement techniques: A comparison of three dimensional body scanning and physical anthropometric methods. Retrieved from http://citeseerx.ist.psu.edu/viewdoc/download?doi=10.1.1.611.48058\&rep=rep1\& type $=$ pdf

Shivaswamy, V., Boerner, B., \& Larsen, J. (2016). Post-Transplant Diabetes Mellitus: Causes, Treatment, and Impact on Outcomes. Endocrine Reviews, 37(1), 37-61. doi:10.1210/er.2015-1084

Silkensen, J. R. (2000). Long-term complications in renal transplantation. J Am Soc Nephrol, 11(3), 582-588.

Sneed, D. B., Birge, S. J., \& Khort, W. M. (1993). Age-related differences in body composition by hydrodensitometry and dual-energy X-ray absorptiometry. Journal of Applied Physiology, 74(2), 770-775.

Snowsill, T. M., Moore, J., Mujica Mota, R. E., Peters, J. L., Jones-Hughes, T. L., Huxley, N. J., . . . Anderson, R. (2017). Immunosuppressive agents in adult kidney transplantation in the National Health Service: a model-based economic evaluation. Nephrol Dial Transplant, 32(7), 1251-1259. doi:10.1093/ndt/gfx074

Stanfill, A., Bloodworth, R., \& Cashion, A. (2012). Lessons learned: experiences of gaining weight by kidney transplant recipients. Prog Transplant, 22(1), 71-78.

Stanfill, A., Conley, Y., Hathaway, D., Cowan, P., Cashion, A., Homayouni, R., . . . Thompson, C. (2015). A Pilot Study of Demographic and Dopaminergic Genetic Contributions to Weight Change in Kidney Transplant Recipients. PLOS ONE, $10(9)$.

Steiber, A. L. (2014). Chronic Kidney Disease. Journal of Parenteral and Enteral Nutrition, 38(4), 418-426. doi:10.1177/0148607114527315 
Tanamas, S. K., Wluka, A. E., Berry, P., Menz, H. B., Strauss, B. J., Davies-Tuck, M., . . . Cicuttini, F. M. (2012). Relationship between obesity and foot pain and its association with fat mass, fat distribution, and muscle mass. Arthritis Care Res (Hoboken), 64(2), 262-268. doi:10.1002/acr.20663

Teixeira, P. J., Sardinha, L. B., Going, S. B., \& Lohman, T. G. (2001). Total and regional fat and serum cardiovascular disease risk factors in lean and obese children and adolescents. Obes Res, 9(8), 432-442. doi:10.1038/oby.2001.57

Toth, P. P. (2004). High-density lipoprotein and cardiovascular risk. Circulation, 109(15), 1809-1812. doi:10.1161/01.Cir.0000126889.97626.B8

Urstad, K. H., Wahl, A. K., Andersen, M. H., Oyen, O., \& Fagermoen, M. S. (2012). Renal recipients' educational experiences in the early post-operative phase--a qualitative study. Scandinavian Journal of Caring Sciences, 26(4), 635-642. doi:10.1111/j.1471-6712.2012.00972.x

Vatanparast, H., Chilibeck, P. D., Cornish, S. M., Little, J. P., Paus-Jenssen, L. S., Case, A. M., \& Biem, H. J. (2009). DXA-derived abdominal fat mass, waist circumference, and blood lipids in postmenopausal women. Obesity (Silver Spring), 17(8), 1635-1640. doi:10.1038/oby.2009.80

Vazquez, G., Duval, S., Jacobs Jr, D. R., \& Silventoinen, K. (2007). Comparison of body mass index, waist circumference, and waist/hip ratio in predicting incident diabetes: a meta-analysis. Epidemiologic reviews, 29(1), 115-128.

Wang, L., Southerland, J., Wang, K., Bailey, B. A., Alamian, A., Stevens, M. A., \& Wang, Y. (2017). Ethnic differences in risk factors for obesity among adults in California, the United States. Journal of Obesity. 1-10. Retrieved from https;//doi.org/10.1155/2017/2427483

Wang, Y., Beydoun, M. A., Liang, L., Caballero, B., \& Kumanyika, S. K. (2008). Will all Americans become overweight or obese? estimating the progression and cost of the US obesity epidemic. Obesity (Silver Spring), 16(10), 2323-2330. doi:10.1038/oby.2008.351

Wang, Y. C., McPherson, K., Marsh, T., Gortmaker, S. L., \& Brown, M. (2011). Health and economic burden of the projected obesity trends in the USA and the UK. The Lancet, 378(9793), 815-825. doi:https://doi.org/10.1016/S0140-6736(11)60814-3

Wang, Z.-M., Pierson Jr, R. N., \& Heymsfield, S. B. (1992). The five-level model: a new approach to organizing body-composition research. The American Journal of Clinical Nutrition, 56(1), 19-28.

Weiner, D. E., Carpenter, M. A., Levey, A. S., Ivanova, A., Cole, E. H., Hunsicker, L., . . . Bostom, A. G. (2012). Kidney function and risk of cardiovascular disease and mortality in kidney transplant recipients: the FAVORIT trial. Am J Transplant, 12(9), 2437-2445. doi:10.1111/j.1600-6143.2012.04101.x

Wen, M., \& Kowaleski-Jones, L. (2012). Sex and ethnic differences in validity of selfreported adult height, weight and body mass index. Ethnicity \& Disease, 22, 72 78.

Whelton, P. K., Carey, R. M., Aronow, W. S., Casey, D. E., Collins, K. J., Dennison Himmelfarb, C., ...Wright, J. T. (2018). 2017 ACC/AHA/AAPA/ABC/ACPM/ $\mathrm{AGS} / \mathrm{APhA} / \mathrm{ASH} / \mathrm{ASPC} / \mathrm{NMA} / \mathrm{PCNA}$ Guideline for the Prevention, Detection, Evaluation, and Management of High Blood Pressure in Adults. $J$ Am Coll Cardiol, 71(19), e127. 
Withers, R. T., Laforgia, J., Pillans, R. K., Shipp, N. J., Chatterton, B. E., Schultz, C. G., \& Leaney, F. (1998). Comparisons of two-, three-, and four-compartment models of body composition analysis in men and women. Journal of Applied Physiology, $85(1), 238-245$.

World Health Organization. (2018). Global Database on Body Mass Index, 2018. Retrieved from http://www.assessmentpsychology.com/icbmi.htm

Yano, Y., Vongpatanasin, W., Ayers, C., Turer, A., Chandra, A., Carnethon, M. R., .. . Neeland, I. J. (2016). Regional Fat Distribution and Blood Pressure Level and Variability: The Dallas Heart Study. Hypertension, 68(3), 576-583. doi:10.1161/hypertensionaha.116.07876

Younespour, S., Rahimi Foroushani, A., Maraghi, E., Rostami, Z., Einollahi, B., Eshraghian, M. R., \& Mohammad, K. (2016). Longitudinal Serum Creatinine Levels in Relation to Graft Loss Following Renal Transplantation: Robust Joint Modeling of Longitudinal Measurements and Survival Time Data. Nephrourology Monthly, 8(5), e39292. doi:10.5812/numonthly.39292

Yu, H., Kim, H., Baek, C. H., Baek, S. D., Jeung, S., Han, D. J., \& Park, S.-K. (2016a). Risk factors for new-onset diabetes mellitus after living donor kidney transplantation in Korea - a retrospective single center study. BMC Nephrology, 17(1), 106. doi:10.1186/s12882-016-0321-8

Yu, H., Kim, H., Baek, C. H., Baek, S. D., Jeung, S., Han, D. J., \& Park, S. K. (2016b). Risk factors for new-onset diabetes mellitus after living donor kidney transplantation in Korea - a retrospective single center study. BMC Nephrol, 17(1), 106. doi:10.1186/s12882-016-0321-8

Zrim, S., Furlong, T. I. M., Grace, B. S., \& Meade, A. (2012). Body mass index and postoperative complications in kidney transplant recipients. Nephrology, 17(6), 582-587. doi:10.1111/j.1440-1797.2012.01621.x 


\section{VITA}

Tara Calico Cherry was born in 1960 in Memphis, Tennessee. Tara earned a Bachelor of Science in Nursing in 1995 from the University of Tennessee and a Master of Science in Nursing in 2008 from Union University. Tara is a certified family nurse practitioner with 5 years of experience in mental health and addictive medicine. She also earned a Master of Business Administration in 2001 from Embry-Riddle University. She will earn a Doctor of Philosophy from the University of Tennessee Health Science Center in December 2018. Her dissertation research, which was funded by Dr. Donna Hathaway's program of research, investigated the effective measures of weight gain of kidney transplant recipients. 